Portland State University

PDXScholar

\title{
Teachers' Critical Reflection in an Equity-Focused Professional Learning Community: a Case Study
}

\author{
Patrizia Lina Mastne \\ Portland State University
}

Follow this and additional works at: https://pdxscholar.library.pdx.edu/open_access_etds

Part of the Educational Leadership Commons, and the Teacher Education and Professional Development Commons

Let us know how access to this document benefits you.

\author{
Recommended Citation \\ Mastne, Patrizia Lina, "Teachers' Critical Reflection in an Equity-Focused Professional Learning \\ Community: a Case Study" (2020). Dissertations and Theses. Paper 5460. \\ https://doi.org/10.15760/etd.7332
}

This Dissertation is brought to you for free and open access. It has been accepted for inclusion in Dissertations and Theses by an authorized administrator of PDXScholar. Please contact us if we can make this document more accessible: pdxscholar@pdx.edu. 
Teachers' Critical Reflection in an Equity-Focused Professional Learning Community:

A Case Study

by

Patrizia Lina Mastne

A dissertation submitted in partial fulfillment of the requirements for the degree of

Doctor of Education

in

Educational Leadership: Curriculum and Instruction

Dissertation Committee:

Micki M. Caskey, Chair

Joanne Cooper

Samuel Henry

Yves Labissiere

Portland State University

2020 
(C) 2020 Patrizia Lina Mastne 


\begin{abstract}
In a culturally diverse world, teachers adopt complex roles to educate a changing student body toward higher standards. They can respond to students' needs and design responsive curriculum by engaging in ongoing learning and improvement. When teachers have time and space for collaboration and reflection, they can learn and improve. Professional learning communities (PLCs) are forums for educators to think deeply about praxis. In this study, the problem of practice is that teachers have had few opportunities to reflect critically within PLCs. The purpose of my study was to describe and explain how critical reflection occurred in an equity-focused PLC within a suburban high school. With situated and transformative learning as a theoretical framework, I conducted a qualitative, collective case study design to explore how three high school teachers' reflections, efficacy beliefs, and practice changed over time. Data sources were surveys, written reflections, and interviews and data analysis included within case analysis and cross-case analysis. Based on my analysis, I identified three themes: (a) teachers feel frustration when PLCs lack structure and reflection, (b) teachers want more productive PLCs, and (c) teachers prefer Critical Friends Groups (CFG) community. To optimize teacher learning, I recommended adjusting policies to fund the more productive and reflective version of PLCs - Critical Friends Groups.
\end{abstract}

Keywords: professional learning community, Critical Friends Group, reflection, critical reflection, situated learning theory, transformative learning, teacher efficacy beliefs 


\section{Acknowledgements}

I would like to thank Dr. Micki M. Caskey for her unwavering support throughout this journey. She guided me through illnesses, lapses in confidence, and bouts of inertia with her steadfast belief in me and my abilities. Her intelligence, strength, and friendship were sustaining, as well as inspirational.

A special thanks to Dr. Samuel Henry, who, not only was my professor and committee member, but first inspired me to apply to the doctorate program with his infectious enthusiasm.

A heartfelt thank you to my distinguished committee members, my many professors, and my supportive classmates.

I am eternally grateful for the infinite love and support of my parents, Gina and James, who have continued to nurture me throughout my life, as much now as when I was a child. They worked tirelessly their entire lives to give me the opportunities to do whatever I wanted in life.

I am forever indebted to my Michael, who has helped me manage my crazy life with constant dedication and love (and occasional sarcasm) for the last 31 years.

I am appreciative of my sisters, Monica and Cassandra, who always answer the call.

Lastly, I am thankful to my brilliant and talented daughter Annalisa, who made my life ineffably richer and was my ultimate motivation to persevere. 
Table of Contents

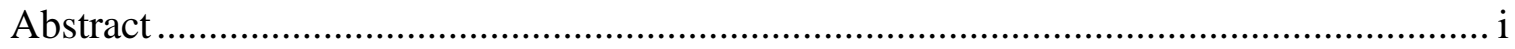

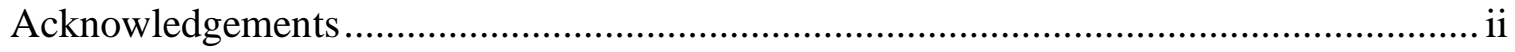

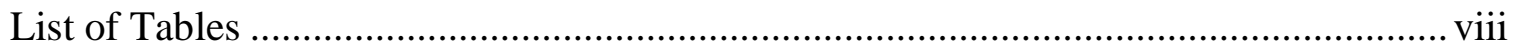

Chapter 1: Problem Statement ........................................................................ 1

Professional Learning Communities Model ...................................................... 3

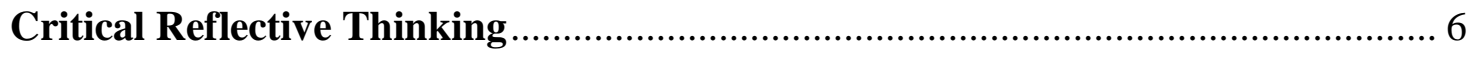

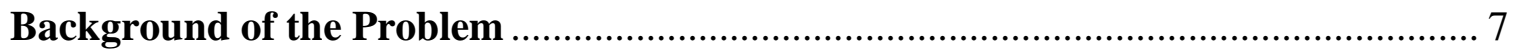

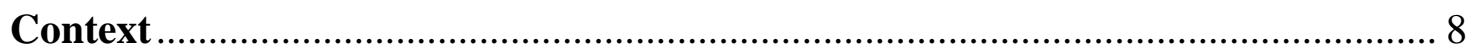

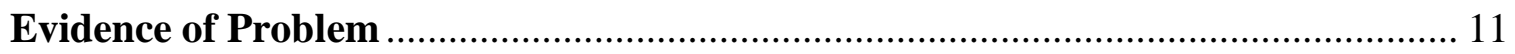

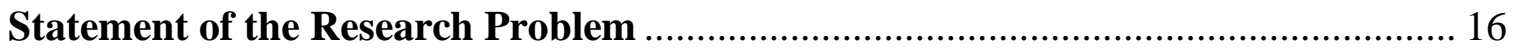

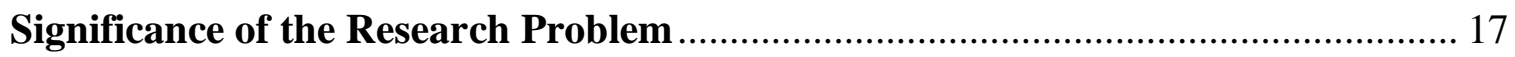

Presentation of Methods and Research Question ............................................... 17

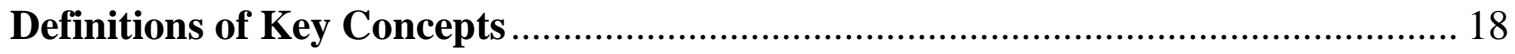

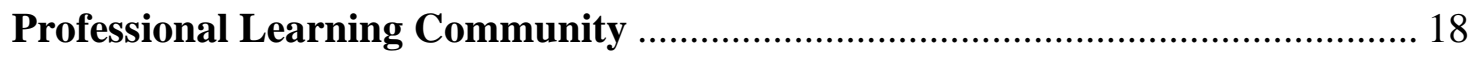

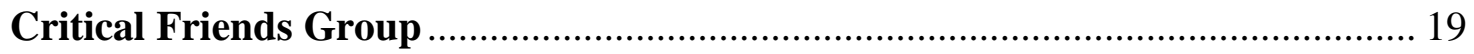

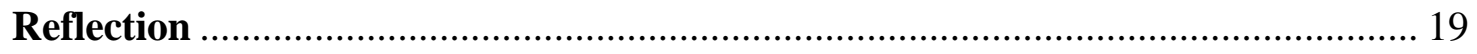

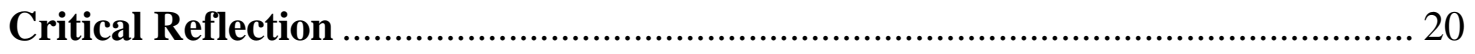

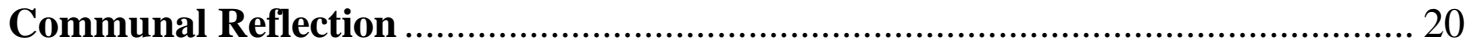




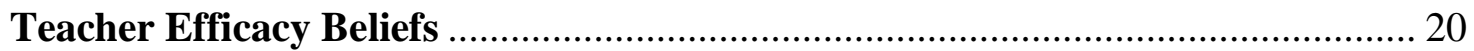

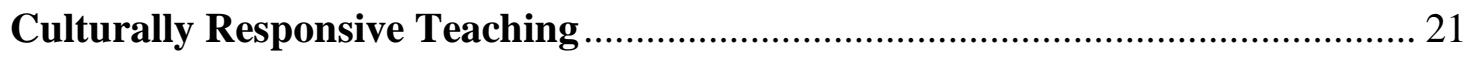

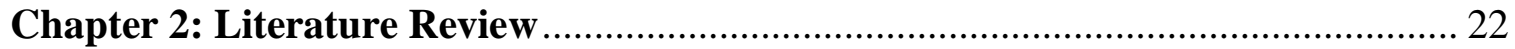

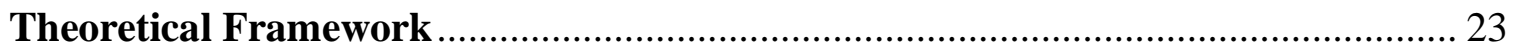

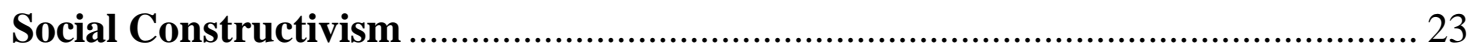

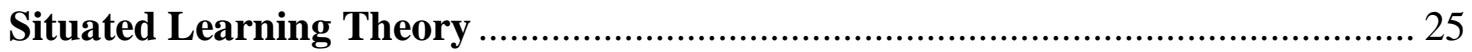

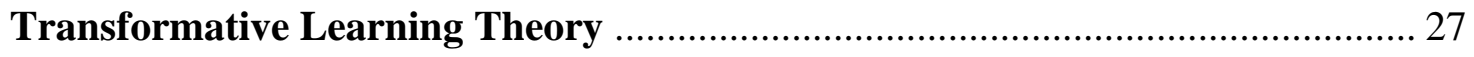

Synthesis of Theoretical Framework ............................................................... 29

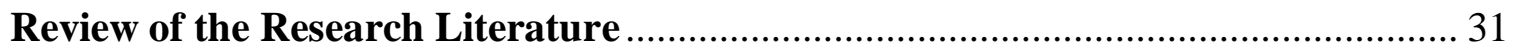

Professional Learning Community ................................................................ 31

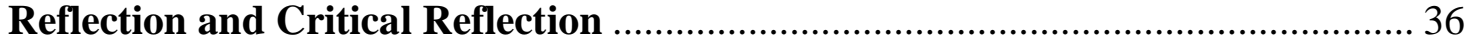

Critical Reflection within a PLC ............................................................... 41

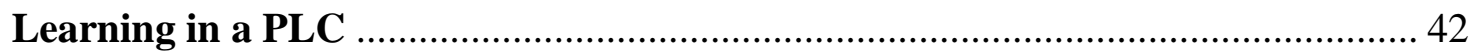

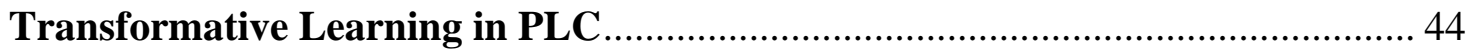

Teacher Efficacy Beliefs, Critical Reflection, and Transformative Learning...... 45

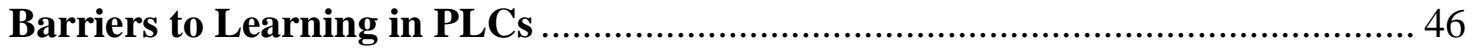

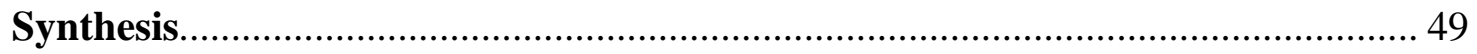

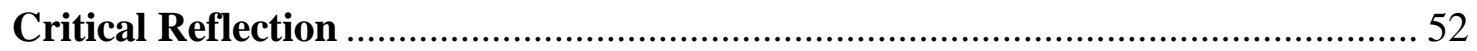

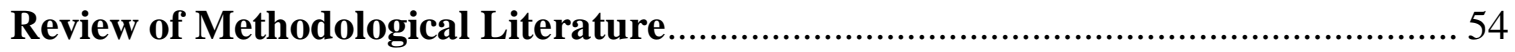


Summary of the Research Literature and Application to My Study ....................... 59

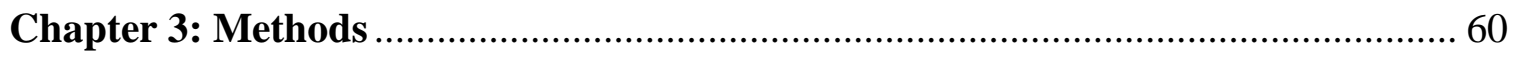

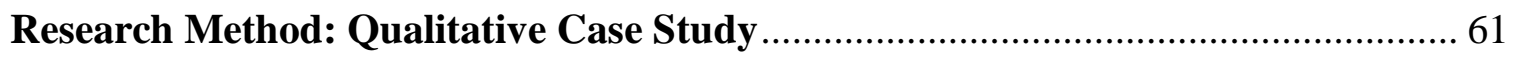

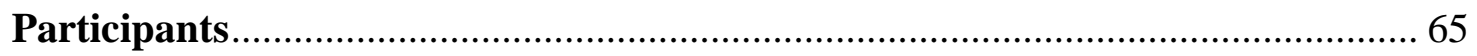

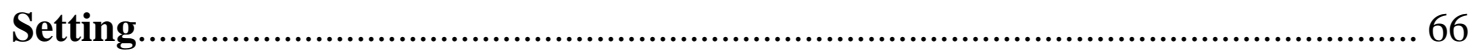

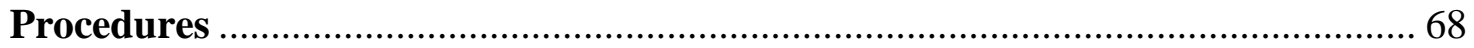

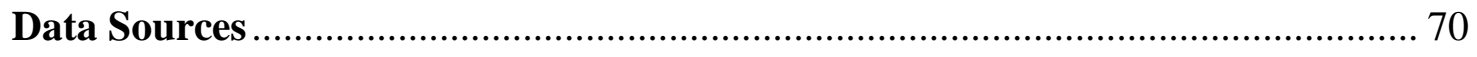

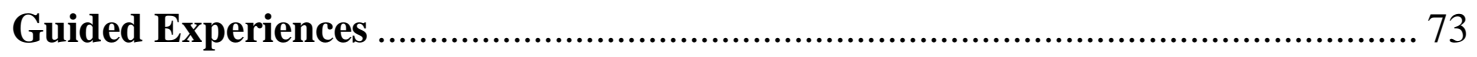

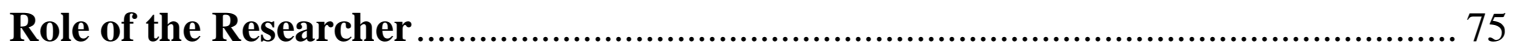

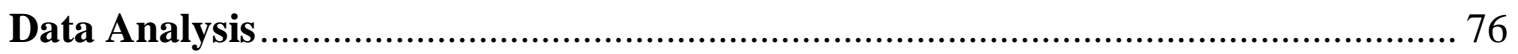

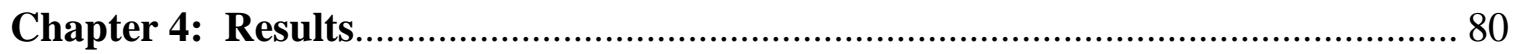

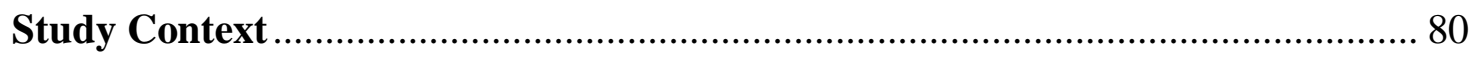

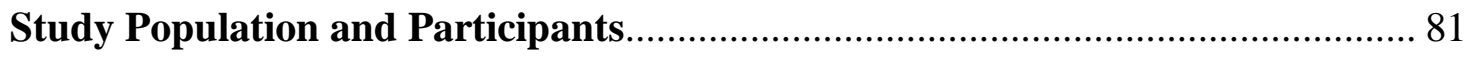

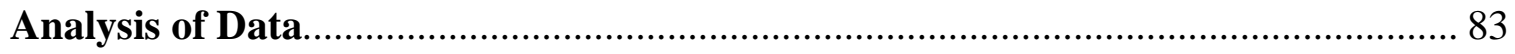

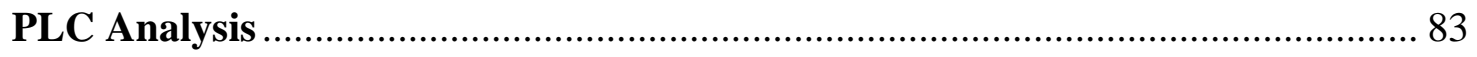

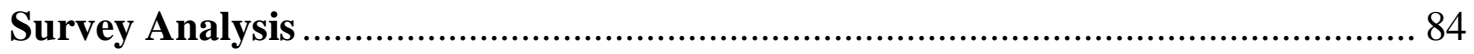

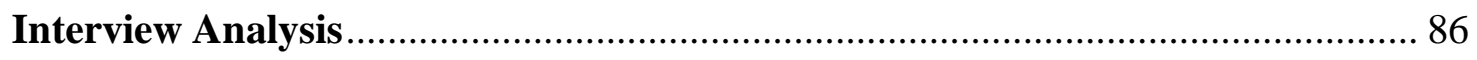

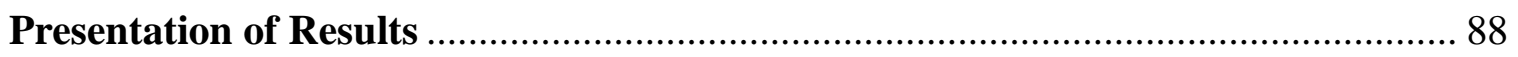

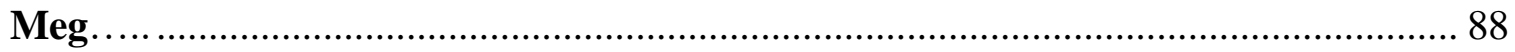


John.

Carmen... 96

Interpretation of Results 101

Cross-Case Analysis

Limitations of the Study . 112

Summary of Results

Chapter 5: Discussion

Major Findings Related to Research Questions 116

Major Findings Related to Research Question 1 116

Major Findings Related to Research Question 2 118

Major Findings Related to Research Question 3

Unexpected Findings.

Situated in the Larger Context 125

Conclusions 128

Teachers Want to Improve 128

Preference for CFG Model of PLCs. 129

Poorly Executed PLCs are Detrimental.

Implications for Action. 130

Recommendation for Further Research 131 
References

Appendix A. Informed Consent Form

Appendix B. Survey of Reflective Practice: A Tool for Assessing Development as a Reflective Practioner Self-Assessment

Appendix C. Culturally Responsive Teaching Self-Efficacy Scale

Appendix D. Larrivee Statement of Permission to Use.

Appendix E. Siwatu Permission to Use Instrument. 160

Appendix F. Written Reflection Exit Prompts 161

Appendix G. Initial Interview Questions 162

Appendix H. Final Interview Questions. 163

Appendix I. Recruitment Document 165

Appendix J. ATLAS.ti ${ }^{\circledR}$ Report 167 
List of Tables

Table 1: Jay and Johnson's Typology of Reflection $\quad 40$

$\begin{array}{ll}\text { Table 2: } & \text { Methods Matrix }\end{array}$

Table 3: $\quad$ Indicators of Change and Typical Examples $\quad 74$

Table 4: $\quad$ Participant Scores from the Survey of Reflective Practice and 85 CRT Self-Efficacy Scale

Table 5: Within Case Patterns and Cross-Case Sub-Themes 102

Table 6: Sub-Themes and Common Codes of Critical Reflection within 104 an Equity-focused PLC for Research Question 1

Table 7: Changes in Teachers' Scores on the Critically Responsive 105 Teacher Efficacy Survey

Table 8: Sub-Themes and Common Codes of Critical Reflection within an Equity-focused PLC for Research Question 2

Table 9: An Example of Learning Activities Linked Qualitatively to Learning Outcomes

Table 10: Change in Teachers' Scores on the Survey of Reflective

Table 11: Meg's Results by Levels of Reflection.

Table 12: John's Results by Levels of Reflection.

Table 13: Carmen's Results by Levels of Reflection.

Table 14: Sub-Themes and Common Codes of Critical Reflection within an Equity-focused PLC for Research Question 3

Table 15: Major Findings Related to Research Question 1

Table 16: Major Findings Related to Research Question 2 


\section{Chapter 1: Problem Statement}

Teaching is hard. In a recent American Federation of Teachers survey, $61 \%$ of educators reported their work as stressful most of the time, compared with $30 \%$ of the public. Teachers further reported experiencing poor mental health 7 to 12 days of a month in contrast to zero days in non-education fields (Mahnken, 2017; Will, 2017).

Teachers experience incredible stress. Moreover, they feel undervalued, overworked, underpaid, and increasingly threatened in today's educational climate (Thiers, 2016). The educational debt-a term indicating the enormous cost of the historical, economic, societal, and moral inequities accumulating disproportionately, for generations, on the backs of Black, Brown, Native, and migrant student populationscontinues to counteract almost any positive teacher action (Ladson-Billings, 2006). Schools struggle persistently to meet the needs of many student subpopulations as evidenced by the glaring differentials in educational outcomes (Hammond, 2015; Ladson-Billings, 2006). To mitigate increasing student disaffection, teachers must rise and meet the needs of students by improving their praxis (Hoffman-Kipp, Artiles, \& López-Torres, 2003; Larrivee, 2000; Tschannen-Moran \& Woolfolk Hoy, 2001). Although poverty, racism, and many other systemic ills plaguing our students are beyond our powers as teachers, by using critical reflection in community, we can (a) discover our hidden bias and agendas, (b) evaluate the worth of what we are doing in the present, and (c) imagine possibilities for the future (Servage, 2008). We can make our little piece of the world better. 
Good teachers have a profound and long-lasting effect on student outcomes (Cochran-Smith \& Lytle, 2009). After controlling for socio-economic status (SES), the quality of the teacher is the biggest factor affecting student achievement (Bakkenes, Vermunt, \& Wubbles, 2010; Darling-Hammond \& Sykes, 1999; Šarić \& Šteh, 2017; Tschannen-Moran \& Woolfolk Hoy, 2001). Teachers have a moral obligation to become life-long learners seeking amelioration of their practice (Campbell, 2003). Although a moral obligation might be open for debate, improving praxis is even becoming a necessity for job security; 42 states nationwide have teacher evaluations tied to student outcomes (Laverick, 2017).

One of the most effective ways to improve teacher practice is by structured, collaborative teacher groups, like professional learning communities (PLCs) (Dufour \& Eaker, 1998). Teachers learn and improve when supported by structures that value their learning, working in forums with other educators and thinking deeply about praxis (Martin-Kniep, 2008). However, forming sustainable, productive PLCs is challenging (Achinstein, 2002; Pancucci, 2008; Servage, 2007). One obstacle identified was teacher disposition; there is a strong correlation between teacher efficacy beliefs and openness to changing and improving practice (Bakkenes et al., 2010, Pancucci, 2008). A key component for increasing teacher efficacy and cultivating their willingness to change is by having educators engage in critical reflection (Servage, 2007). Critical reflection, alone and in community, promotes deep questioning of beliefs, assumptions, bias, and practice, and can lead to transformative learning and growth (Brookfield, 1995; Larrivee, 
2000; Šarić \& Šteh, 2017; Servage, 2008). PLCs may provide teachers a perfect avenue to engage in regular, consistent critical reflection.

\section{Professional Learning Communities Model}

School districts across the nation are adopting a PLC model as a unifying framework for defining the teaching and learning that should characterize the environment (Servage, 2008). In its simplest form, a PLC is a forum in which educators co-construct learning for improving their practice for students' academic benefit. Ideally, the PLC model becomes part of the school culture by engaging in regular, ongoing collaborative inquiry and analysis focused on the commitment of continuous improvement and learning for all (DuFour, 2004; Martin-Kniep, 2008; Servage, 2007).

PLCs are in vogue because of their potential to enhance both teacher and student learning and to transform educational practice (Martin-Kniep, 2008; Servage, 2008; Valli, 1994; Wood, 2007a). PLCs can be key agents in shaping teacher's norms, knowledge, and in sustaining change (Martin-Kniep, 2008). Although schools around the nation are implementing PLCs, issues regarding sustainability and dysfunction have emerged (Achinstein, 2002; Servage, 2008). Enduring tensions persist regarding the time teachers must engage in meaningful, collaborative learning in a climate of standards and assessments, as well as value conflicts about professional autonomy, power, and culture (Achinstein, 2002; Westheimer, 2008). Furthermore, there is a gap in the research linking PLCs to improved student outcomes, especially for Black, Brown, Native, and English language learners (Banks et al., 2005; Ladson-Billings, 2006). 
Acknowledging schools' efforts to adhere faithfully to the tenets of successful implementation of PLCs, the question remains: why is there scarce data linking PLCs and consistent, improved student outcomes as a result? I contend that the transformative change promised from learning communities is inconsistent because of the lack of attention given to the vital role of teacher reflection in learning. Rigorously examining and reflecting on attitudes, beliefs, and practices, in conjunction with community challenges, opens the door to new ideas and knowledge. Removing critical reflection from the PLC process impedes teacher learning, and ultimately student academic progress.

Locally, in Riverton School District (pseudonym), the system-wide implementation of PLCs is a key strategy used to attain the district goal of increasing teacher learning through collaboration and improving student outcomes. The school district mandates district-wide PLCs to examine teacher practice and student data. The stated purpose of PLCs is to encourage teacher learning, collaboration, and leadership to serve students more effectively by increasing achievement through better teaching. The goal of the district's PLC model is to provide a continuous improvement system that regularly tracks the progress of individual students and empowers teachers to work collaboratively toward efficacy. The district intends that the system-wide implementation of PLCs allow teachers to share and refine effective teaching practices, with students as the ultimate beneficiaries.

I have been a member of several PLC groups during my career working in the Riverton School District (RSD). From 2005 to 2008, I participated in, and occasionally 
coached, a Critical Friends Group (CFG); it was a transformative experience for me. A CFG is a specific, more formal type of PLC consisting of approximately 8 to 12 educators meeting, voluntarily, at least once a month for about two hours; group members commit to improving their practice through collaborative learning (Caskey \& Carpenter, 2012). During meetings, group members bring forth student artifacts and teacher-practice concerns for communal, critical examination and discussion. In the days prior to the meeting, members correspond via email suggesting prospective topics for analysis. Then, the teachers negotiate the agenda collaboratively and equitably. The sessions follow one of many protocols (i.e., formats designed to ensure equal participation, safe environments, dialogue, and critical reflection). Among colleagues, I listened to, questioned, challenged, and defended beliefs, issues, and concerns related to our teacher practice. The meetings were two hours long to allow adequate space and time for member voice, reflection, and analysis. Often, the groups continued to meet over the course of several years. Eventually the more formal and lengthier CFGs fell out of favor with district decision-makers and streamlined learning communities, PLCs, replaced them.

My subsequent experiences with learning teams over the past 20 years have been far less successful. The shorter, less formal PLCs replacing CFGs have rendered equitable, critical dialogue and reflection nearly nonexistent. Like the dysfunctional PLCs described in the literature, protocols are no longer tools to advance teacher learning, but rather tools to advance a district agenda (Wood, 2007a). Space and time for 
critical reflection within the PLCs has been inconsistent and poorly developed, to the detriment of teacher learning.

The district presented the PLC model as an ongoing opportunity for teachers and administrators to engage conjointly in reflective inquiry and action that would lead to transformative learning and ultimately increased achievement and educational equity for all students. PLC meetings were to be 90 minutes in length and fit into the school schedule. School leaders (i.e., administrators and teacher leaders) directed the teachers to bring student products, data, and information to explore to the meetings. Teachers seemed to accept the directives passively and support the idea that students' test scores were the most important method of determining future action and policy. Administrators and teacher leaders and appeared to acknowledge opposing opinions, but then moved on to another comment.

\section{Critical Reflective Thinking}

Teachers cannot fully realize the benefits of PLCs and experience transformative change until the important role of critical, reflective thinking is given its full due (Servage, 2007). Learning and growth may not take place without a safe environment for constructive meaning making to occur; the learner's individual reflection on new, negotiated meanings allows for sense making that is inclusive, permeable, critically reflective, and integrative of experience (Mezirow, 2000; Servage, 2007). Educators must engage in a power analysis of what is important, how it is important, why it is important, and who says it is important (Brookfield, 2000). Deep, reflective thought and dialogue in community can unveil and challenge hegemonic assumptions. Critical 
reflection allows for new, negotiated understandings of knowledge and practice and alternative perspectives and growth (Achinstein, 2002; Brookfield, 2000; Servage, 2007).

A PLC that seeks continuous improvement of effective practices for educators appears to be a solid unifying principle. However, does the learning community allow for corroboration or nullification of beliefs and practices through vigorous, communal reflective analysis and critique? If not, is either learning or community occurring in the spirit intended? I contend that for learning and community to occur, thoughtful critical reflection must be intentional, jealously guarded, and rigorously promoted.

The purpose of my study was to explain and describe teachers' critical reflection in an equity-focused PLC within a suburban high school. I examined teacher efficacy beliefs, as self-reported, over the course of their participation in the PLC through surveys, samples of reflective writing, and interviews. I took the position that purposeful reflection opportunities within the PLC platform could encourage deeper thinking and learning, and subsequently impact teacher efficacy beliefs and student centered and culturally responsive pedagogy in the classroom. In this study, I highlighted conditions conducive to teacher learning and growth within learning communities, as well as suggested areas for improvement, thereby strengthening PLCs, teachers' learning and promoting more consistently positive student outcomes.

\section{Background of the Problem}

The problem of practice is that teachers have few opportunities to reflect critically in PLCs. While literature on the implementation of a PLC in schools include teacher reflection as an essential component (Martin-Kniep, 2008), research is scarce on how to 
foster a reflective environment. Although PLCs could theoretically provide the time and space for critical reflection, few studies have reported if and how critical reflection occurs within the setting of a PLC. Critical reflection must take place for teachers to examine their beliefs, bias, and assumptions (Brookfield, 2000; Servage, 2008). Through critical reflection, teachers can confront their preconceptions and self-generating mental habits, avoiding remaining "trapped in unexamined judgements, interpretations, assumptions, and expectations" (Larrivee, 2000, p. 294). Furthermore, Mezirow asserted that through critical reflection of our own and others' assumptions one can experience transformative insight, which then undergoes justification through communal discourse (2000). Unexamined and unchallenged bias and assumptions regarding the increasingly diverse student populations may contribute to the disparity in achievement between demographic subgroups.

\section{Context}

In this section, I describe the context of my problem of practice. I include demographics of the school district and their implementation of the early-release professional days. Last, I describe the background of the study school.

The district. Riverton School District has a large and diverse population. The district's students of color profile are more than 50\%, almost a quarter of which are Hispanic. The state identifies nearly $40 \%$ of the student population as holding an impoverished socio-economic status as determined by enrollment in the free and reducedprice lunch program. Riverton relies on community taxes and bond measures to supplement federal and state financial contributions and, therefore, has a complex and 
dependent relationship with the school board. Due to a perennially unstable state funding system, Riverton School District must make considerable organizational sacrifices to integrate approximately a \$15 million-dollar deficit.

In 2016, the district, with equity and democracy at the forefront, released its new goals. Its primary goal ensures that each student earns a high school diploma and is ready for post-high school learning. The district commits to providing learning teams for its teachers to increase and improve student learning outcomes. RSD places an emphasis on community relationships, as well as building safe and inclusive connections with students and families. The district strives to impart the knowledge and skills students need for an evolving world. To foster a collaborative, learning organization, RSD also introduced early-release Wednesdays.

Early-release Wednesdays. The district's strategic plan, developed with community involvement and support, identified teacher collaboration as the key strategy for ensuring teacher learning and individual student growth and instituted Early-Release Wednesdays. Students now have a weekly 90-minute early release, allowing teachers the time to collaborate and participate in professional development consistently.

The school. North River High School (NRHS) is a suburban school with approximately 1500 students. Its population has a $30 \%$ sector of students eligible for free or reduced-price lunch, 25\% students of color and 5\% English Language Learners (ELL). The school serves grades 9 through 12 . The students generally take four core classes and two to three electives during a seven-hour, even-odd day rotation. The school also offers an International Baccalaureate (IB), Dual Credit (with the college), and Dual 
Language (DL) diploma. The school's mission statement places a strong emphasis on developing compassionate and responsible citizens of the school, community, and the world.

The guiding principle of the school is to maintain a safe, inclusive, and equitable environment for students. The teachers enjoy a collegial, friendly staff in a wellmaintained newer building and personable administrators. Many of the teachers have worked at the same school since its inception. The principal is new, but generally well received for his even-handedness. The school is in a period of transition with many new teachers and support staff, as well as a rapidly changing demographic. When a new high school opened recently, the district restructured the neighborhood-school boundaries, and the NRHS student demographics shifted abruptly. Because the school sat in an upscale neighborhood, the population was overwhelmingly White, educated, and upper middle class. Now the school is adjusting to an influx of traditionally disenfranchised populations: students of color, language learners, immigrants, and the economically disadvantaged. Population analysis projects the trend to continue for the next decade. The principal dedicates resources to ensuring equity and works to maximize opportunities for all students. The school attempts to keep strong relations with the community and parent-volunteers, although it is struggling to better communicate and include Latino and immigrant families.

Teachers are under the supervision of the administrators, but primarily for procedural duties and goal setting. On early-release Wednesdays, all teachers attend at least one content-specific PLC, one staff PLC, and two special interest PLCs each month 
by mandate.

The school board and subject-specific teacher-cadres determine the contentspecific curriculum for the district. National directives (No Child Left Behind Act (NCLB) and Every Student Succeeds Act (ESSA) require schools to have a School Improvement Plan (SIP) in place to track progress. For example, in the case of NRHS, the SIP states that students scoring at the proficient level on the state assessments would increase $10 \%$ by the end of the 2017 school year. In comparison to district averages, NRHS has higher SES level and higher 2016 scores in both reading and math. District analysts expect the figures to change precipitously as the repercussions of the boundary changes take effect.

In line with district goals, the schools' school improvement plan revolves around learning teams focused on equity for under-served populations especially, collaboration, and learning. Every stage of the learning cycle allows space for questions and critical reflection. The purpose of my study was to explain and describe teachers' critical reflection in an equity-focused PLC within a suburban high school.

\section{Evidence of Problem}

Schools have enacted more than 30 years of reforms, including teacher collaborative learning teams like PLCs, since A Nation at Risk (National Commission on Excellence in Education, 1983) decried the dismal state of education and yet there has been little change in the academic disparity in learning outcomes between student subpopulations (Darling-Hammond \& Sykes, 1999). Further, the number of Black, Brown, Native, and linguistically diverse students in public education rose to $51.5 \%$ from 
$42 \%$ in the last decade, yet $82 \%$ of teachers in the United States are White (National Center for Education Statistics, 2016).

If teachers are to educate a much more diverse student body toward higher standards and adopt more complex roles in a technological world, then teachers need enormous flexibility and skill (Šarić \& Šteh, 2017). Teachers must know how to design curriculum and adapt their teaching to respond to students' understandings, experiences, and needs, as well as their family and community contexts. This task cannot be prepackaged or teacher-proofed (Darling-Hammond \& Bransford, 2005).

Modern teachers must not only deliver curriculum, but they must also take on new roles in the classroom: diagnostician, challenger, model, activator, monitor, evaluator, and reflector of student learning (Šarić \& Šteh, 2017). Teachers must be able to analyze and adapt to a complex and diverse student body in specific social, cultural, and political contexts (Liu, 2017). With such a wide range of responsibilities, it is little wonder that, after controlling for SES, teacher quality is the single most important predictor of positive student outcomes (Ferguson, 1991). Therefore, it is more important than ever for teachers to engage in ongoing learning and improvement.

Organized learning environments, like professional learning communities and peer coaching, elicit better teacher learning activities (e.g., experimenting, changing techniques), as well as better teacher learning outcomes (i.e., increased positive emotions and greater change in teacher efficacy beliefs) than informal collegial conversations (Vermunt, 2014). 
Wood (2007b) noted that societies build upon foundations of knowledge and teachers needed to be at the center of the knowledge construction. Schools are the obvious site for that effort and fortunately, PLCs are now ubiquitous across the United States. It is within PLCs that teachers co-construct knowledge, enhancing teacher learning (Martin-Kniep, 2008; Servage, 2008; Wood, 2007b). In this context, reflection is essential to connect work to past and future practice (Martin-Kniep, 2008). Further, teachers need the space and time to examine implicit assumptions of their practice to effect real change (Servage, 2007).

Teachers need to refine and reform their practice consistently to learn and grow professionally (Thompson \& Zeuli, 1999; Tyack \& Cuban, 1995). Although PLCs can be a beneficial and useful conduit for teacher development (Caskey \& Carpenter, 2012; Servage, 2008; Westheimer, 2008; Wood, 2007b), they may fall short of their intended purpose for many reasons. The most common causes identified for unsustainable or underperforming PLCs have been a preset, top-down agenda; a lack of trust between participants; apathy or resistance among members; the absence of rigorous, critical conversations around assumption and bias; and the lack of time and space for reflection (Achinstein, 2002; Martin-Kniep, 2008; Overstreet, 2017; Rusch, 2005; Servage, 2007; Westheimer, 2008).

In discussing PLCs, Servage (2008) emphasized a specific characteristic to a fruitful learning community - the inclusion of collaborative work that involves critical reflection and problem solving in authentic contexts of daily teaching practices. Including collaborative problem solving and critical reflection ensures that teachers' 
work is productive - not reductive. In other words, by deflecting focus from the technical aspects of practice, the PLCs avoids becoming "performance training sects" ( $p$. $15)$.

Effective teaching is more than skills and strategies. It also includes a deliberate philosophical and ethical code of conduct (Larrivee, 2000). Teachers must contend with the fact that schools are complex, social, and political entities. In PLCs, teachers can have the platform to reflect critically on their actions and the contexts in which they occur. Teachers can accept responsibility for ensuring that they uncover and challenge bias and assumptions that are contrary to democracy, justice, inclusion, and equity (Servage, 2008). By discussing and reflecting on critical matters (i.e., social justice) in community, teachers can remove the barrier of avoiding uncomfortable truths (Brookfield, 2000). After all, the purpose of public schools is to ensure all children access to common ideas, skills, and knowledge to become productive adults in society (Berliner \& Biddle, 1995).

Engaging in critical reflection can mitigate the conflict and discomfort of addressing ideological differences and tensions among group members (Achinstein, 2002; Westheimer, 2008). Successful school cultures for productive PLCs foster responsibility for individual ideas, tolerance for the views of others, and a capacity to negotiate differences (Meier, 2000). Yet, "seldom are (teachers) in relations where there is sufficient trust to allow for conflict, differences in opinion, and risk-taking in ways that lead to personal growth" (Attard, 2012, p. 205). When teachers squelch differences of opinion — overtly or through covert 'let us just get along' messages — the group may 
maintain stability; however, they also may pay the high price of impeding ongoing inquiry and transformative change (Achinstein, 2002).

In a well-constructed PLC, the community dissects and discusses assumptions, biases, and beliefs, negotiating and constructing new meaning that is more egalitarian, inclusive, and democratic (Feldman \& Fataar, 2014; Owen, 2014; Servage, 2009; Westheimer, 2008; Zeichner, 1993). For transformative learning to occur within the PLC, educators must engage in a power analysis of what is important, how it is important, why it is important, and who says it is important (Brookfield, 2000). Critical reflection and dialogue in community can unveil and challenge hegemonic assumptions, allowing for new, negotiated understandings of knowledge and practice and alternative perspectives and growth (Šarić \& Šteh, 2017). PLCs are the contexts that cultivate deep understanding, insight, innovation, and inspiration and allow its members to create opportunities to internalize and articulate tacit knowledge derived from their daily work (Martin-Kniep, 2008).

Educational literature is replete with articles on the necessity of reflection for learning and its many benefits (Achinstein, 2002; Brookfield, 2000; Dewey, 1933; Mezirow, 2000; Servage, 2008; Westheimer, 2008). However, almost no studies describe reflection in situ; that is, how, or if, opportunities for reflection exist in PLCs and what effect, if any, it has on teacher learning and practice (Owen, 2014). For this reason, I conducted a small pilot study to gauge teachers' efficacy beliefs about teacher learning in their PLCs. I found that teachers were not using the PLCs as places of teacher learning; they were gaining knowledge outside of their time in the PLC. I also noted that the PLCs 
within the schools were neither places of learning nor examples of reflective practiceinstead diluted to little more than a prescribed training session.

Better schooling and better teachers may likely result from the steady, reflective effort of practitioners (Tyack \& Cuban, 1995). Ultimately, the people with the problems also have the key to the solution. "Teachers are works in progress and must remain committed and open to inquiry, exploration, reflection, and transformative change" (Ayers, 2001, p. 141). The choice is the teacher's as to how to see the students and the world and which directives to support or resist (Ayers, 2001).

\section{Statement of the Research Problem}

The problem of practice is that high school teachers have few opportunities to reflect critically, even in their learning communities. Although PLCs are an organizational structure that can, theoretically, provide the time and space for critical reflection, few studies have reported if and how critical reflection occurs within the setting of a PLC (Achinstein, 2002; Van Lare \& Brazer, 2013). The purported goal of school PLCs is to elicit teacher learning; yet, there is scant evidence in the literature of what goes on within a PLC in situ. As a participant observer in this study, I explored the inner workings and dynamics of an equity-based PLC. Specifically, I was interested in (a) when critical reflection occurred, (b) if and how teachers learned, and (c) if and how teacher efficacy beliefs and behaviors changed post-PLC experiences.

The purpose of my study was to explain and describe teachers' critical reflection in an equity-focused PLC within a suburban high school. I was curious to learn if and how teachers used critical reflection. To explore teachers' critical reflection, I also 
needed to examine teacher efficacy beliefs and if and how teacher classroom behaviors changed, as self-reported through surveys and interviews, over the course of their participation in the PLC. It is my position that purposeful reflection opportunities within the PLC platform encourages deeper thinking and learning, influencing teachers to increase culturally responsive pedagogy in the classroom. This study explores conditions conducive to reflection, learning, and growth within learning communities, and suggests areas for improvement, thereby strengthening PLCs, teachers' learning and promoting more consistently positive student outcomes.

\section{Significance of the Research Problem}

PLCs are popular because of their potential to enhance both teacher and student learning and to transform educational practice (Martin-Kniep, 2008; Servage, 2008; Valli, 1994; Wood, 2007a). This study brings to light the inner world of a PLC: if and how critical reflection occurred; if and how participants discussed, ignored, or suppressed their biases and assumptions; and how teachers perceived the process and its usefulness regarding their practice. The use of critical reflection is imperative for teachers to advance their thinking and actions in today's schools (Peters, 2016). This study has the potential to benefit theory and practice by contributing to the knowledge base about the importance and effect of critical reflection during PLCs and adding to the literature regarding PLC conditions conducive to teacher learning.

\section{Presentation of Methods and Research Question}

I employed qualitative cross-case case study design for my research. A case study allowed me to delve deeply into the in situ procedures, discourse, and participant 
interactions within a PLC. Through surveys, written reflections, interviews, and field notes, I explored teachers' perceptions of the PLC process. Participants were novice through veteran high school teachers who reported different levels of efficacy within an equity-based PLC from a suburban high school.

The following research questions guided my work:

1. How does critical reflection unfold within an equity-focused PLC without prompting?

2. In what way does critical reflection influence teachers' beliefs?

3. How does critical reflection influence teachers' perceptions of their practice?

\section{Definitions of Key Concepts}

In this section, I provide definitions of key terms related to my problem of practice. These terms include professional learning community, reflection, critical reflection, teacher efficacy beliefs, and culturally responsive teaching.

\section{Professional Learning Community}

A PLC is a forum in which educators co-construct learning individually and collectively for improving their practice and the goal of bringing about systemic, transformative change (Martin-Kniep, 2008; Servage, 2007). Members of the community share a set of values and norms that they co-construct within the context of their work, fostering interdependence. Goals of PLC include improving teacher practice, thereby improving student learning; creating a culture of intellectual inquiry open to difficult conversations; reducing alienation; and pursuing social justice, democracy, and a communal way of life (Westheimer, 2008). 


\section{Critical Friends Group}

A Critical Friends Group (CFG) is type of PLC consisting of 5-12 educators "who commit to improving their practice through collaborative learning and structured interactions (protocols) and meet at least once a month for about two hours" (National School Reform Faculty [NSRF], n.d., Para 1). Unlike general PLCs which often focus on test standards for improving student performance, CFGs use "specific protocols and activities to promote best practices" for teaching and improving student achievement (NSRF, n.d., Para 3). A protocol is a structured set of guidelines used by the attendees to encourage meaningful communication, problem-solving, and learning. Further, trained teacher-coaches, along with various protocols, guide the discussions and the meetings. Group members take turns bringing a practice-based quandary to the table for public deliberation. CFGs honor and prioritize time for deep reflection, reveal solutions to complex dilemmas, build trust between colleagues, reduce teacher isolation, foster equity, and change school culture (NSRF, n.d., Para 4).

\section{Reflection}

Dewey (1933) differentiated between reflection and reflective thinking. In the broadest form, thinking is any perception that passes through our minds. Reflection, however, is a consecutive ordering of thoughts that becomes a thread of thought. He further described reflection as having a "note of invention" (p. 5) discrete from basic observation of the senses. Dewey reserved his highest regard for an optional step in deeper thinking, reflective thought, or thinking "active, persistent, and careful consideration of any belief or supposed form of knowledge in light of the grounds that 
support it, and the conclusions to which it tends” (p. 9). For this study, I use the terms reflection, reflective thought, and reflective thinking interchangeably.

\section{Critical Reflection}

For reflective thinking to rise to the level of critical reflection requires another criterion. According to Brookfield (2000), "The individual must engage in some sort of power analysis" of an experience or situation, becoming aware of oppressive structures (p.126). Mezirow (2000) added that critical reflection should also include an attempt to uncover and identify personal, hegemonic assumptions and bias. He asserted that critical reflection can lead to transforming frames of references, or beliefs and assumptions. Brookfield noted, "Although critical reflection often comes from autobiographical analysis, its full realization occurs only when others are involved...(it) is an irreducibly social process" (pp. 140-141). When viewed through the eyes of others.one gains deep insight into one's values, beliefs, and practices.

\section{Communal Reflection}

Communal reflection is deep or critical reflection carried out in a group setting, whereby members of a community share their experiences and reflections aloud among peers. Making thoughts and ideas public allows for analysis, critique, and challenges to the validity and appropriateness of actions, assumptions, and beliefs within a given context.

\section{Teacher Efficacy Beliefs}

A teacher's efficacy belief is a judgment of their capabilities to bring about desired outcomes of student engagement and learning, even among those students who 
may be difficult or unmotivated. Teacher efficacy is both context and subject-matter specific. A teacher may feel very competent in one area of study or when working with one kind of student and feel less able in other subjects or with different students, so it is context and subject-matter specific (Tschannen-Moran \& Woolfolk Hoy, 2001).

\section{Culturally Responsive Teaching}

Culturally responsive teaching (CRT) is the use of the cultural knowledge, prior experiences, and frames of reference of traditionally underserved populations, making learning and assessing more relevant and effective (Gay, 2002). Culturally relevant pedagogy (CRP), culturally appropriate instruction, and culturally compatible instruction are other terms referring to CRT (Siwatu, 2007).

In the preceding section, I provided brief definitions of key concepts to orient the reader to my study as I explored how critical reflection unfolded in the PLC. In Chapter 2, I present my theoretical framework, a review of the research literature, and the methodological literature related to my study. 


\section{Chapter 2: Literature Review}

The problem of practice was that high school teachers have few opportunities to reflect critically, even within their PLCs. Although PLCs are an organizational structure that can, theoretically, provide the time and space for critical reflection, few studies have reported if and how critical reflection occurs within the setting of a PLC (Achinstein, 2002; McComish \& Parsons, 2013). The purported goal of school PLCs was to elicit teacher learning which ultimately benefits the practice, the students, and the school; yet, scant evidence in the literature indicated what goes on within a PLC in situ. As a participant observer in this study, I explored the inner workings and dynamics of an equity-based PLC. Specifically, my research questions were:

1. How does critical reflection unfold within an equity-focused PLC without prompting?

2. In what way does critical reflection influence teachers' beliefs?

3. How does critical reflection influence teachers' perceptions of their practice? The purpose of my study was to explain and describe teachers' critical reflection in an equity-focused PLC within a suburban high school. I found limited literature about understanding and fostering teacher learning; fewer research studies examined how teachers learn at work (Bakkenes et al., 2010; Darling-Hammond \& McLaughlin, 2011; Overstreet, 2017). This study addressed the need for research focusing on the relationship between critical reflection, teacher efficacy beliefs, teacher learning, and teacher practice, while providing detailed descriptions of the complex phenomena of reflection in a PLC. I used case study for my research design to describe and explain the 
processes of reflection in PLC, including teacher critical reflection and learning experiences.

I studied the phenomenon of critical reflection in a PLC and its influence on teacher learning within the framework of socioconstructivist, situated learning, and transformative learning theories. In this chapter, I present relevant research on PLCs, learning, and critical reflection highlighting their interdependence.

\section{Theoretical Framework}

Learning is complex, fraught with metaphor, assumption, and context. Many different theories have attempted explanations of how learning occurs (Bereiter, 1994; Cobb, 1994; Dewey, 1910; Ernest, 1993; Fenwick, 2000; Lave, 1996; von Glasersfeld, 1991). The theories are not perfect, and I believe no one theory can explain all aspects of learning.

People make meaning from their own point of view and from the social construct of the context surrounding them, laden with assumptions, bias, and politics (Brown, Collins, \& Duguid, 1989; Cobb, 1994; Servage, 2008). The hegemonic power structure maintains that its values are everyone's values (McLaren, 1994). Given my stance that learning is always in context and typically among people, my theoretical framework includes three theories: social constructivism theory, situated learning theory, and transformative learning theory.

\section{Social Constructivism}

Social constructivism, like its progenitor constructivism, espouses the view that learners either adapt to or accommodate novel situations (Cobb, 1994). Learning is the 
result of disequilibrium in the learner's experience (Dewey, 1933). Social constructivists understand learning as a viable organization, an accommodation, or assimilation of the new information in the learner's existing tableau. Learners then re-organize their constructs through accommodation or assimilation to eliminate perturbations. Social constructivists view signs, symbols, and language as a means by which learners express and communicate their thinking (Cobb, 1994). Social constructivism views individuals as inextricably bound with society, engaged in conversation about their experiences of the physical reality. The humanly constructed reality shifts to fit the ontological (truth or seminal knowledge of the world) reality. This paradigm adopts relativist ontology; a socially constructed, shared world that we can perceive but we have no absolute knowledge of (Ernst, 1994). From this perspective, the mind is the individual-in-socialcontext and learning is the interactive process of enculturation in a community, or a PLC for this study. Language, specifically persons in discussion with others, is central to an individual's learning (Ernest, 1994).

Critique and implications. Von Glaserfeld (1991) asserted learning as occurring when an individual interacted with other members of a community in viable or nonviable ways. However, constructivism did not provide any role for the desire to learn (Fenwick, 2000). In earlier theories of constructivism, context was important but separate, although the modern program has assimilated aspects of the sociocultural perspective (Bereiter, 1994). Critics pointed out that not all learning in community was beneficial and unguided learners may reinforce negative practices (Fenwick, 2000). 
Critical theorists, however, dismissed the apolitical position of sociocultural perspectives (Fenwick, 2000).

\section{Situated Learning Theory}

Situated learning theory (Lave, 1996; Lave \& Wenger, 1991) is a social constructivist perspective embedded in situ. In other words, cultural, economic, political, and structural forces act on and around members of a community and determine their actions and behaviors (Lave, 1996). People co-construct knowledge from within a community, much like a river that changes as it ebbs and flows within the environment. The knowledge is in constant flux, an entity created by the community — and its cultural values, assumptions, norms, activities — and the individual (Fenwick, 2000). The community of truth is a collection of knowers; the expert becomes more equal, a knower around a subject (Fenwick, 2000). The activity is an integral part of the learning and allows for the development of knowledge (Brown et al., 1989; Lave, 1996). As a form of cultural apprenticeship, learning through embedded activity using the social context is imperative (Brown et al., 1989; Lave, 1996). Situated in and central to the social context of PLCs is the communal nature of cognition, learning, and meaning (Stein \& Imel, 2002). It is the consistent interplay between theory, participants, and activities that allows for valuable interpretations capable of explaining the dialectic relationship that exists between the social structures (e.g., the PLC) and human agency (e.g., the will and action of the teachers). Knowledge construction is never-ending, inventive, and entwined in doing (Fenwick, 2000). Because context is critical, this learning theory appeals to the democratic spirit of co-participation that neutralizes much of the social inequity that can 
occur in education (Lave, 1996). Proponents of situated learning theory defined language and knowledge as culturally mediated (Brown et al., 1989). Knowledge is the inventive intersection of context, language, experience, sense making, and what is relevant to the setting (Sfard, 1998). As an example, participants' experience in practical contexts solidifies knowledge and the understanding of tools (i.e., science nomenclature). The learner and the community co-construct their understanding of the knowledge base and cultural beliefs (Brown et al., 1989). The learning is "intentional and is in response to situations requiring an action at the personal, organizational, or community level" (p. 95) making the knowledge gained explicit (Stein \& Imel, 2002). To learn, then, is to know how to participate meaningfully in chosen practices.

Critique and implications. Critics contended that situated learning theory was weak regarding the transfer of knowledge, because knowledge was neither centered in any one learner nor fixed (Anderson, Reder, \& Simon, 1996; Fenwick, 2000). This meant a person's reflective thinking, memory, or ability to transfer knowledge to a novel situation played no significant role. Other critics pointed out that unsupervised participants could reinforce negative or undesirable strategies and practices that were limiting or subversive to the community (Anderson et al., 1996). Proponents of situated learning theory have addressed these concerns by reiterating: learning was a process of active and discursive enculturation; community synergistically innovated solutions and ideas; confronted ineffective ideas; and communally negotiated new, justifiable knowledge (Cobb, 1994). Knowledge, like language, was accessible from its use and 
context, perpetually evolving and under construction, and negotiated by the society (Brown et al., 1989, Sfard, 1998).

\section{Transformative Learning Theory}

Transformative learning theory refers to mindful, reflective, communicative, discursive, and democratic learning (Brookfield, 2000; Mezirow, 2003). Individually and in community, learners parsed and discussed assumptions, bias, and habits, constructing meaning that was more egalitarian, inclusive, and democratic (Mezirow, 2003). Reflection was necessary for, but not synonymous with, transformative learning (Brookfield, 2000). In other words, the learner could reflect in community on the hidden agenda and power structures embedded within an educational practice yet decide to maintain one's bias or fail to act by changing one's practice. Transformative learning occurred only when a belief system or practice underwent a substantial revision and reframing, not simply a repackaging of old ideas (Brookfield, 2000). However, few studies have linked the presence of transformative learning in PLCs (McComish \& Parsons, 2013).

Critique and implications. In discussing PLC, Servage (2008) emphasized a specific characteristic to a fruitful learning community: the inclusion of collaborative work that involved critical reflection and problem solving in authentic contexts of daily teaching practices. Fidelity to the real work of teachers and their students maintained the relevance of PLCs, whereas a focus on the technical aspects of practice changed learning communities into "performance training sects" (p. 15). According to Martin-Kniep (2008), enduring changes could not emerge when the focus was solely on technical work 
handed down from above. However, administrators often asked teachers to inquire critically about their practice while instructed incongruously to ignore the contextual issues that shaped their practice. To accommodate the prescribed agenda, administrators suppressed broader ideas and concerns (Servage, 2007). Studying effective practices without regard to social, cultural, and ethical contexts still had value as teacher learning, but it was neither exemplary of collaborative practice nor transformative (Servage, 2008). The real work of professional learning communities - improving teacher and student outcomes in their socio-political contexts with collaborative reflection and dialoguecould provide the necessary platform for transformative change and learning (McComish \& Parsons, 2013).

The vital role of critical, reflective thinking ushers in the transformative learning and change that exemplifies the best PLCs (McComish \& Parsons, 2013; Servage, 2007). Learning and growth would not take place without a safe environment for constructive meaning making to occur; the learner's individual reflection on new negotiated meanings allowed for sense making that was inclusive, permeable, critically reflective, and integrative of experience (Mezirow, 2000: Servage, 2007). Educators needed to engage in a power analysis of what was important, how it was important, why it was important, and who says it was important (Brookfield, 2000). Deep, reflective thought and dialogue in community unveiled and challenged hegemonic assumptions. Critical reflection allowed for new, negotiated understandings of knowledge and practice and alternative perspectives and growth (Achinstein, 2002; Brookfield, 2000; Servage, 2007). 


\section{Synthesis of Theoretical Framework}

In the preceding paragraphs, I described the theoretical framework of social constructivism, situated learning, and transformative learning theories that guided my research. Situated learning theory is a social constructivist perspective embedded in situ. This theory positions knowledge as the inventive intersection of context, language, experience, sense making, and what is relevant to the setting (Sfard, 1998). In PLCs, teachers co-construct and negotiate meaning, through collaborative discourse, inquiry, and reflection, relevant to their praxis. Embedded in their context, teachers carry with them their combined assumptions and shared experiences to navigate new meaning. I also set my study within the transformative learning frame. Transformative learning

refers to the mindful, reflective, discursive interaction in community between learners as they deconstruct bias and habits to construct new meaning that is more egalitarian, inclusive, and democratic (Brookfield, 2000; Mezirow, 2000). Transformative learning, the implicit goal of PLCs, is impossible without communal, critical reflection to uncover and identify hegemonic suppositions and ineffectual solutions seen through the eyes of others. PLCs have the potential to be an ideal conduit for teacher improvement and transformative learning. Promoted as a democratic forum for consistent and continuous improvement, a venue for rich discussion of practice, and a trusted community for close examination of beliefs, PLCs can fulfill all these expectations or none of them. Synthesis of the PLC Literature

In this section, I offer a synthesis of the literature about professional learning communities (PLCs). I focus specifically on how PLCs can be an effective form of 
professional development for teachers, the essential features of PLCs, and the opportunity for critical reflection.

Successful implementation of PLC requires teacher reflective thinking as an integral component (Jones \& Thessin, 2002; Overstreet, 2017). Martin-Kniep (2008) asserted that without the disposition of reflection, work seemed disconnected from the future or the past. Rodgers (2002) also illustrated Dewey's commitment to public discussion of the utility of professional work done in community and the necessity in hearing one's reflective thoughts aloud to reveal its strengths and weaknesses.

Allowing teachers to collaborate with one another solely on prescribed tasks cannot produce the change education needs. Teachers need time and space to examine and reflect critically on their practice within the PLC to more likely produce authentic change. While the shortage of time and resources are not inconsequential, Servage (2007) argued that the most formidable barrier to effective collaboration might be the "collective consequence of our individual weaknesses, our individual choices, our individual insecurities, our individual fear of change, and our individual quests for power" (p. 71). Critical, reflective thinking and dialogue allowed practitioners the constructive medium for addressing, confronting, and resolving individual and collective tensions that otherwise inhibit PLC from bringing about the change that it intends. Although other concerns can impede constructive PLC, this study focused on issues more directly affected by individual or group action, specifically individual and collective critical reflection. Next, I offer a review of the research literature regarding my research study. 


\section{Review of the Research Literature}

In this section, I provide an overview of the historical context of PLCs and the research literature regarding the influence and importance of reflection and critical reflection specifically during professional learning communities. I also include a literature summary of the link between critical reflection, learning, and importantly, transformative learning.

\section{Professional Learning Community}

In its simplest form, scholars defined a professional learning community (PLC) as a forum in which members co-constructed learning individually and collectively for the purpose continuously improving their practice with the expectation of transformative change (Martin-Kniep, 2008; Servage, 2007; Overstreet, 2017). Hord (2004) described PLCs as "communities of continuous inquiry and improvement" (p. 1). These learning communities shared common characteristics: supportive leadership, shared vision, collective learning, application of the learning, and shared personal practice (Hord, 2004). The teachers (and other staff) fostered interdependence and shared a set of values and norms within the context of their work (Calderwood, 2000; Westheimer, 2008). The cyclical learning model stemmed from the learning organizations of the 1980s corporate world (Senge \& Lannon-Kim, 1991). DuFour and Eaker (1998) later adapted the corporate terminology to accommodate education and christened the professional learning community (Vangrieken, Meredith, Packer, \& Kyndt, 2017). However, Dewey (1916) wrote that professional work revealed its strengths and weaknesses when teachers 
debated and discussed its attributes publicly, perhaps the first allusion to educational learning systems.

PLCs picked up momentum at the start of the millennium when No Child Left Behind prompted many districts across the nation to return professional development to the purview of the teachers in individual schools (Vescio, Ross, \& Adams, 2008). Westheimer (2008) described six main goals of professional learning communities:

1. Improving teacher practice to improve student learning,

2. Creating a culture of intellectual inquiry, open to difficult conversations,

3. Increasing teacher capacity to lead through collaborative leadership,

4. Mentoring novice teachers,

5. Reducing alienation, and

6. Pursuing social justice, democracy, and a communal way of life.

Along with the goals of PLC, a careful review of the literature indicates the five key characteristics PLCs theoretically share: (a) common values and vision that provide a framework for decision making, (b) collective responsibility for student learning, (c) reflective professional inquiry, including frequent critical reflective dialogue examining teacher practice and contextual dilemmas, (d) collaboration and interdependence among colleagues, and (e) group and individual learning is promoted (Stoll et al., 2006).

Owen (2014) posited that although the literature indicated general agreement on the important characteristics of successful PLCs, which of the factors the researchers emphasized changed dramatically and could account for the differential degrees of impact PLCs had on teacher and student learning and outcomes (Owen, 2014; Vescio et al., 2008). 
In her case study exploring the experiences of teachers and teams involved in PLC, Owen (2014) employed purposive sampling to select three innovative schools in which to study 58 participants. Her methods included surveys, interviews and focus groups during one school year. She used narrative vignettes of three teachers as representative of the findings from the different schools. Findings revealed the PLCs shared specific characteristics: common values about students, collaboration focused on data, teacher inquiry, and responsibility for collegial learning. Data revealed that collaborative and personal reflection and dialogue nurtured teacher learning. More than $90 \%$ of the teachers indicated changes in their practice and increased student outcomes. Owen noted that teachers highlighted the collegial learning culture as powerful. Owen concluded with thoughts on key processes of sustainable PLCs: (a) forming group identity and norms of interaction, (b) navigating divergent views, (c) negotiating the essential tension between student and teacher learning as the focus, and (d) communal responsibility for individual growth of colleagues. Owen asserted that PLC work needs to move beyond conviviality, through navigating and negotiating divergent viewpoints before significant benefits in teacher growth can occur.

Lave and Wenger (1991) posited while developing the situated learning model that learning is a process that takes place in the context of specific communities of learning. Wood (2007a) wrote that societies built on the foundations of knowledge needed teachers to be at the center of knowledge construction; and therefore, schools were the obvious sites for that effort. Through intentional adaptation or transformation of 
practices, communities were at the core of learning when concomitantly committed to the goals previously outlined.

Servage (2008) emphasized a more specific characteristic to a fruitful learning community: the inclusion of collaborative work involving inquiry and reflective problem solving in authentic contexts of daily teaching practices. "Collaborative inquiry and reflective practice are the language and sustenance of PLCs" (Martin-Kniep, 2008, p. 6). However, collaborative inquiry and reflection need not be in a common space for a productive learning community as noted by King (2011).

In her case study, King (2011) explored reflection and learning in unlikely spaces — social media and virtual communities — as professional development. Simultaneously addressing the difficulty of common time or space for professionals to gather, King used a case study design to investigate the learning and reflection of a primary participant who engaged in an online blog community focusing on a mental disorder. The community included professionals, nurses, students, and people diagnosed with the disorder. King followed the public blogs (and private chats), and twitter accounts of her consenting participants for 14 months, as well as analyzing journal entries and member-checking for validity. King's analysis of data indicated that community developed among participants, the primary participant benefitted from the online professional development experiences, and the blogs afforded the primary participant with opportunities for transformative learning. In addition, she concluded that in this case, self-directed learning is an effective way to keep pace with new knowledge and a professional community provides the space for the learning to occur. This study 
elucidated the importance of quality, trusting community over the physical proximity of members.

As noted previously, focusing solely on technical aspects of practice reduced learning communities into "performance training sects" (Servage, 2008, p.15). In a study focusing on teachers' perceptions of their data-driven PLCs, Sims and Penny (2014) explored impediments to successful learning communities in a large suburban high school. Using qualitative case study method, the researchers collected data through structured interviews with six participants and classroom observations of 12 teachers. Combined with field notes from three additional observations of the PLC meetings, the researchers triangulated for external validity. Analysis indicated little positive outcome for overall school improvement. Teachers found the PLCs with their constricted focus and metrics, interfered with needed collaboration, specifically concerning the lack of comprehensive discussion about contextual praxis. Sims and Penny emphasized the importance of trust and collaborative discussions, concluding that productive PLCs should not limit the scope to single metrics, as numbers alone rarely convey everything needed for informed decision-making. Mezirow (2000) posited that the additional space allowance for critical reflection would allow teachers to question the validity of assumptions regarding competitive grading or high-stakes testing and eventually reject the premise outright, turning to alternate forms of measuring a student's learning gains.

In Learning among Colleagues, Westheimer (2008) also described some of the pressing challenges that confronted the building of successful professional community. The first obstacle was the isolated nature of most educators' work. 
Ironically, although teachers most frequently cited their isolation as the main barrier to learning and collaboration, they were resistant to making their practice public (Tyack \& Cuban, 1995; Westheimer, 2008). "The autonomy of isolation afforded teachers is often so deeply entrenched; it is difficult to dissuade them from it psychologically, as well as physically" (Westheimer, 2008, p. 770). Another issue in forming sustainable PLCs was time- - there was never enough time in a school day. Master schedules were often contrary to common meeting times among teachers. Further, the tensions within communities stemming from power differentials or incongruent views lead to unproductive PLCs or even the dissolution of the group. Many PLCs ignored, glossed over, or rejected discussing the difficult topics of core beliefs, equity, or "the overarching democratic goals of education" (Westheimer, 2008, p. 776). However, dissension could have created the framework for learning and the revitalization of communities (Achinstein, 2002).

Although other concerns impeded constructive PLCs, not the least of which were resources and systemic support, this study limits discussion to issues more directly affected by individual and group action. Despite these common pitfalls of professional learning communities, the goals and values encompassed by the concept are worth fighting for within our educational systems.

\section{Reflection and Critical Reflection}

John Dewey, in his seminal work How We Think (1933), differentiated between plain thinking and reflective thinking. In the broadest sense, according to Dewey, thinking was any perception that passed through our minds and reflection was a 
consecutive ordering of thoughts that became a thread of thought. He further described reflection as having a "note of invention" (p. 5) discrete from basic observations of the senses. Reflection was purely imaginative or encompassed a transmitted belief, such as "sinners are excluded from heaven" (p. 5). This thought became reflexive thought, because the person sought no additional inquiry or foundational information. Dewey reserved his highest regard however for an optional step in deeper thinking; reflective thought, which was "active, persistent, and careful consideration of any belief or supposed form of knowledge in light of the grounds that support it, and the conclusions to which it tends" (p. 9). Furthermore, the origin of reflective thought was confusion, doubt, or disequilibrium (Dewey, 1910). Dewey's ideas about reflective thought and reflection laid important groundwork for the use of reflection in educational settings.

Almost a century later, educational literature was replete with articles on reflection and its many benefits for teaching (Steeg, 2016). For example, Zeichner and Liston (1996) outlined five levels of reflection that occur in teaching: (a) rapid reflecting, which is automatic, (b) repairing, when one adjusts practice to meet needs, (c) reviewing, which includes thinking, discussing, or writing about practice, (d) researching, which necessitates systematic and sustained thinking over time, and (e) retheorizing and reformulating, when one critically examines pedagogy and praxis through the lens of academic theories.

However, a solid definition of the reflection remained nebulous. Citing the difficulty in assessing, discussing, or practicing an ambiguously defined act, Rodgers (2002) simplified the philosophical texts of Dewey to the benefit of practitioners and 
researchers. Rodgers distilled Dewey's numerous writings on reflection and its purposes into four criteria:

1. Reflection was a meaning-making process.

2. Reflection was a systematic way of thinking.

3. Reflection needed to happen in community.

4. Reflection needed the participant to be open to growth.

Rodgers, through Dewey's lens, traced the path from an experience to meaning (learning) via one's spontaneous interpretation of the event, the identification of an issue that was discomforting, the generation of explanations, the construction of hypotheses, and the testing of the hypotheses. Communally reflecting mitigates the risk of bias and selfdelusion (Mezirow, 1990; Rodgers, 2002; Laverick, 2017). Public critical reflection interrupts and reconstructs" human beliefs through "skeptical questioning and imaginative speculation" and can correct inconsistent or irreconcilable knowledge constructions (Achinstein, 2002; Brookfield, 1995; Fenwick, 2008; Mezirow, 2000). This disequilibrium leads the reflective interpreter to refine, revise, or reject meanings. In emphasizing the cyclic nature of reflection and reflective thinking, Rodgers (2002) also highlighted the scientific foundation of Dewey's ideas. Nevertheless, as in science, each new refinement, revision, or rejection of a "solution" lead to new and different lenses, as well as differing degrees of dissonance. This process was learning. Another important theme in the reflection literature was the co-mingling of individual and collaborative reflective processes, as Jay and Johnson (2012) pointed out: 
Reflection is a process, both individual and collaborative, involving experience and uncertainty. It is comprised of identifying questions and key elements of a matter that has emerged as significant, then taking one's thoughts into dialogue with oneself and with others. (p. 76)

Not only is internal and external reflection intertwined here, but similarly inextricably entwined are reflective dialogue and personal reflection. Jay and Johnson distilled the typology of reflection further to three dimensions: (a) descriptive, when the matter for reflection is determined, (b) comparative, a reframing of the subject at hand through the perspective of others or new research, and (c) critical, when consideration of the implications of the new perspective occurs. Although reflection is challenging to concretely define, the importance of critical reframing through the lenses of others is clear.

In a 2017 qualitative study, Laverick explored the understanding and use of reflection among secondary teachers from a wide range of schools. Laverick's grounded theory research relied on Rodgers's (2002) four criteria of reflection to form the theoretical framework for his study: the need for thoughts and reflection to be lifted from the subconscious to one's awareness for deeper thought (meaning-making); the need for systematic and rigorous inquiry to occur (scientific inquiry model); the need for collaborative reflection (community); and the need for valuing the intellectual growth and learning of all (transformative learning). Using both pre and post surveys and interviews over the school year, the five participating teachers provided ample, rich data for the analysis. Laverick found that the teachers' responses shared a strong understanding of 
the meaning-making aspect of reflection and described continual meaning-making throughout their daily practice. However, he noted that in almost every situation the reflections never proceeded to the second stage of rigorous inquiry nor did they usually include community. Laverick noted that by limiting their understanding and use of reflection, its full power, including personal and communal growth and learning, did not occur. Laverick concluded by suggesting that the staff learn Rodgers's pillars of reflection to improve their understanding of reflection and its many benefits when used in its totality.

Along with Rodgers's (2002) pillars of reflection, Jay and Johnson (2012) captured the most inclusive typology to describe reflection (see Table 1). Divided into three dimensions, their organization of reflection serves as a tool for the types of questions that lead to the different levels of the term to guide students and new teachers toward reflective practice.

Table 1

Jay and Johnson's Typology of Reflection

Dimension Definition Typical Questions

Descriptive

Describing the matter for reflection.

Reframing the matter for reflection

Comparative considering others' perspectives, research, etc.
- What is happening

- Is this working? For what and for whom?

- How do I know? How am I feeling?

- What do I not understand?

- What are alternate views of what is happening? Who is served and not served?

- How can I improve what is not working?

- How do people directly involved describe and explain the matter? 
Having considered the implications of

Critical the matter, establishing a renewed perspective.
- What are the implications of the matter when viewed from these alternative perspectives?

- Given these alternatives, and my own morals

- What is the deeper meaning of what is happening in terms of public democratic purposes of schooling?

- What does the matter reveal about the moral and political dimension of schooling?

Adapted from Jay, J. K., \& Johnson, K. L. (2002). Capturing complexity: A typology of reflective practice for teacher education.

\section{Critical Reflection within a PLC}

Since the 1980s, school districts nationwide have fostered teacher community to improve teacher practice and student learning (Achinstein, 2002). Educational systems have had a great deal of interest in the use of PLC as a forum in which members, with shared values and common work, co-constructed learning for improving their practice and enhancing teacher and student learning in tandem (Caskey \& Carpenter, 2012; Fullan, 2006; Jones \& Thessin, 2015; Martin-Kniep, 2008; Servage, 2007; Westheimer, 2008; Wood, 2007b). Studies of professional communities revealed a pattern of initial success followed by dissolution of the group (Achinstein, 2002; Printy, 2008). While communities highlighted shared values, reaching consensus in a diverse community was messy (Servage, 2007). However, conflict also created the context for learning and renewal of communities (Achinstein, 2002). Calderwood (2000) posited that people build the strength and resilience of a community through their attention to their differences and dissensions. Decisions, mediated meanings, and goals of the community 
emerged through bargaining (e.g., articulating preferences) and negotiating (e.g., wielding power) (Achinstein, 2002; Bolman \& Deal, 2008; Calderwood, 2000; Morgan, 2006; Pancucci, 2008). Learning occurred when organizations reached negotiated consensus through critical reflection and discourse (Achinstein, 2002). Importantly, critical reflection was necessary for constructing new ideas and contexts that incorporated multiple perspectives, as well as for connecting teacher work to past and future practice (Martin-Kniep, 2008; Servage, 2008). Therefore, as an essential component to productive PLC, administrators need to provide adequate space and time for teachers to examine and reflect collaboratively on their assumptions and biases regarding practice (Darling-Hammond \& McLaughlin, 2011; Linder, Post, \& Calabrese, 2012; Servage, 2007).

\section{Learning in a PLC}

The brain's ability to receive and process information while considering prior experience and knowledge is known as double-loop learning (Morgan, 2006).

Recognizing incongruences and novel situations followed by questioning and altering responses is (double-loop) learning (Meizrow, 2000). Likewise, organizations can learn from the past and create new meaning. When people in an organization generate new insight to change overarching behaviors and routinely question norms and values, then real change and (organizational) learning can occur (Achinstein, 2002; Morgan, 2006). Individuals or systems of individuals (e.g., PLCs), could receive, interpret, and act on information. The question remains, was the community learning? Learning entails the community being self-aware, questioning and checking its interpretations and actions. 
Are the group norms and values compatible with new interpretations of information? Knowledge construction needs to be in context and must have ongoing communal critique, in which people test the knowledge against their assumptions and biases (Liu, 2017). A group of people hanging together, congenially talking about issues and agreeing on actions without challenging the status quo, is a clique - not a

PLC. Communities of learners explore, reflect, and question the information, the interpretations, and even the beliefs of each other (Achinstein, 2002).

Although misguided community leaders may argue, persuade, and manipulate the PLC for their own interests and ideas by suppressing dissent, they often maintain stability and the status quo in the short term (Achinstein, 2002). Consensus and accepting shared vision and goals without critical reflection and inquiry could become a form of groupthink (Morgan, 2006) as unsupervised participants could reinforce undesirable strategies and practices that were subversive to the community (Morgan, 2006). Instead, by nurturing critical reflection, learning could be a process of active and discursive enculturation, innovating solutions, confronting ineffective ideas, and communally negotiating new, justifiable knowledge (Cobb, 1994). Indeed, Rusch (2005) asserted, "Organizational learning occurs in cultures that foster persistent interaction during change efforts to achieve collective learning and shared meaning" (p. 85). Critical reflection in community could act as a counterbalance to unintended consequences. In fact, deep, reflective thought and dialogue in community could unveil hegemonic assumptions and allow for negotiated understandings of knowledge and practice 
(Brookfield, 2000). In this way, participants could take ownership of their new perspectives.

\section{Transformative Learning in PLC}

Developing skills in critical reflection requires sustained practice, intellectual engagement, and purpose. The purpose of reflection in education should lead to transformative changes in the person reflecting and their surrounding world (Liu, 2015). Critical reflection should lead to transformative learning that alters problematic frames of reference, making the educator more inclusive, discriminating, open, reflective, and emotionally able to change (Mezirow, 2003, pp 58-59). However, reflecting does not guarantee critical reflection and critical reflecting does not guarantee transformative learning. To ensure an outcome of transformative learning from critical reflection requires teachers to act or change their practice based on prior reflections, and then to analyze the effect of the act on student learning, and thereafter to refine and repeat as needed (Liu, 2015).

PLCs provide an egalitarian platform for educators to gather, discuss, and collaborate on matters of praxis, promoting teacher learning and positive student outcomes. PLCs appear to offer the solution to many educational woes, but often fall short (Servage, 2008; Sims \& Penny, 2015). As King (2011) noted, learning is not so much the changes in what we know, but rather the changes in how we know. For critical reflection to bring about transformative learning (and action), it is essential to uncover, question, and challenge the conditions that "undermine democracy and perpetuate social injustices" (p. 66). Schools should be places free of the conditions that in the outside 
world rob some populations of their freedom, dignity, and hope (Servage, 2008). For the school to undergo transformative change, teachers must first undergo transformation (Darling-Hammond \& Sykes, 1999). If PLCs intentionally included critical reflection with a goal of transformative learning, teachers could move away from social communities focused on technical mastery and toward collaborative analysis and reflective discourse on student learning within the context of educational purpose through the lens of an equitable and just society (Servage, 2008). Through critical reflection and transformative learning, teachers could mitigate the demoralizing societal attitudes that prefer making the teacher the answer to all the problems of education, which only deflect attention away from under-resourced schools and crippling poverty (Cochran-Smith \& Lytle, 2009).

\section{Teacher Efficacy Beliefs, Critical Reflection, and Transformative Learning}

A teacher's efficacy belief is a judgment of their capabilities to bring about desired outcomes of student engagement and learning, even among those students who may be difficult or unmotivated (Tschannen-Moran \& Woolfolk Hoy, 2001). A teacher's sense of efficacy contributes to teacher effectiveness in many ways. Teacher's with high self-efficacy beliefs are more likely to: (a) implement new learning to the classroom, (b) seek alternative methods to reach students, (c) experiment more with instructional materials, (e) respond more productively to stressful classroom situations, and (f) produce superior student achievement across a broad range of subjects (Bray-Clark \& Bates, 2003). To increase efficacy beliefs, schools can foster a culture of critical reflectionengaging teachers in discussions of assumptions about student capacities and needs- 
which could lead to transformative change, and PLCs provide the perfect platform for the cultivation of collegial trust, reflection, and learning (Awkard, 2017).

In an action research project, Awkard (2017) and her colleagues implemented a culture of teacher reflection in a large urban middle school, to combat disproportionately low Black and Latinx students' scores on achievement metrics. Suspecting teachers' low efficacy beliefs was at least partially to blame, Awkard began the study by assessing the teachers' efficacy beliefs by guiding them to collaboratively reflect using the Reflective Action Protocol - a cyclical process of reflection through self-assessment, observations, discussions about the observations, discussions and reflections about instruction and student learning. Awkard's findings indicated increased teacher efficacy beliefs linked with higher student achievement and teacher transformative growth. Awkard concluded by asserting that nurturing reflection and guiding teachers to critically reflect on and change biased assumptions and practices is difficult but necessary work that needs to be based on a foundation of trust and non-judgement. A well-structured PLC can be the place where teachers safely explore transformative ideas and acquire the habits of mind required for growth and learning (Servage, 2008).

\section{Barriers to Learning in PLCs}

Organizations, like PLCs, have the potential to be places where people learn from one another and co-create new and expansive patterns of thinking (Senge \& Lannon-Kim, 1991), however, they often are not learning places. In fact, schools face enduring obstacles to develop, maintain, and sustain productive PLCs (Achinstein, 2002; Servage, 2007; Westheimer, 2008). Key issues preventing organizational learning (i.e., changing 
school culture into a place of continuous improvement through the PLC structure) include power differentials and politics among players, teacher resistance, and ideological differences, often grouped together under the umbrella term of micropolitics. Micropolitical theory focuses on individual differences, diversity, conflict, negotiations of consensus between group members, and informal and formal uses (and abuses) of power to achieve community goals (Achinstein, 2002; Servage, 2007). Studying the processes individuals and the community use to achieve goals is central to understanding the inner dynamics of a PLC (Achinstein, 2002; Graham, 2007; Smeed, Kimber, Millwater, \& Ehrich, 2009).

Other studies have attributed PLC failure to powerful players manipulating the group through external policies (e.g., No Child Left Behind) or district pressures (e.g., Race to the Top requisites) (Servage, 2007; Wallerstein \& Duran, 2003; Westheimer, 2008). As an example, in an era of high-stakes testing and accountability and decreasing school monies, administrators and teacher-leaders often limited the scope of PLC to raising test scores (Louis \& Marks, 1998; Westheimer, 2008). By mandating the topics for analysis - a breach in the seminal covenant of productive PLC formationadministrators invalidated teachers' experience, knowledge, and professionalism, as well as preventing important discourse around the validity of test scores as a measure of student growth (Fullan, 1995; Martin-Kniep, 2008; Servage, 2008; Westheimer, 2008). Only by grappling with the conditions of the problem firsthand could one think (Dewey, 1910). To advance instructional effect, teachers had to struggle with personal pedagogical questions and arrive at their own resolutions (Fishman \& McCarthy, 1998). 
Another barrier to successful implementation of PLC was general teacher resistance to change, specifically when they were not involved in the decision-making (Hoffmann-Kipp et al., 2003; Smeed et al., 2009; Tyack \& Cuban, 1995). Practitioners needed to be involved in defining problems, devising context, and developing local culture-specific solutions (Darling-Hammond \& McLaughlin, 2011; Louis \& Marks, 1998; Tyack \& Cuban, 1995). Naturally, teachers hold different values, beliefs, experiences, and information. The poor management of ideological differences and tensions among group members was a significant barrier to a fruitful, self-sustaining PLC (Achinstein, 2002; Westheimer, 2008). Successful school cultures for productive PLC fostered responsibility for individual ideas, tolerance for the views of others, and a capacity to negotiate differences (Meier, 2000). Squelching differences of opinionovertly or through covert 'let's just get along' messages - means that the maintenance of stability was at the price of ongoing inquiry and transformative change (Achinstein, 2002).

Organizational learning only occurred with persistent, collective interactions; intense communication; and reflective thinking, dialogue, and inquiry (Louis \& Marks, 1998; Rusch, 2005). The transformative change required a metaphorical lifting of the blinders and breaking of the chains that kept organizations and individuals blind, bound, and defensive in the face of change (Mezirow, 2000; Morgan, 2006; Servage, 2007). Conflict was natural to experience in a collaborative community seeking substantial change in school norms, culture, and practices (Achinstein, 2002; HoffmannKipp, 2003; Westheimer, 2008). However, embracing differences by making time and 
space for critical reflection and dialogue mitigated the negative consequences of dissent, fostering a strong democracy of diverse people capable of acting toward a common purpose (Achinstein, 2002; Kahne, 1994; Servage, 2007).

Frequent, organized opportunities for collective, intense dialogue, persistent inquiry, and reflective thinking assuaged power differentials, teacher resistance, and ideological differences between group members (Rusch, 2005). Without professional, reflective communication related to shared norms, values, and beliefs about the nature of teaching and collegial experience, school transformation was unlikely (Leithwood \& Jantzi, 2008; Rusch, 2005).

\section{Synthesis}

In this section, I offer a synthesis of the research literature about professional learning communities (PLCs). I focus specifically on how PLCs can be an effective form of professional development for teachers, the essential features of PLCs, and the opportunity for critical reflection.

PLC for professional development and teacher learning. PLCs are a more effective form of professional development. Teachers' own experiences and practices provide much of the learning content that can inspire them to change their thinking and practice (Linder et al., 2012). PLCs provide ongoing, often site-based, collaborative learning spaces with their colleagues. Because trust is fundamental to building the relationships required for successful and sustainable PLCs, the community space allows for deep inquiry and critical reflection about bias, assumptions, and values, coinciding 
with communal challenges and critiques, unlocking the potential of transformative learning.

In a year-long pilot study by Chauraya and Brodie (2017), researchers sought to identify PLC activities that led to teacher learning and transformative change in praxis. With Chauraya as the facilitator, four mathematics teachers from one high school met for two-hours weekly, engaging with various learning activities: (a) analyzing student test errors, (b) identifying student conceptual holes, (c) reflection on teachers' (own) mathematical understanding, (d) designing and teaching lessons on specific content, and (e) jointly reflecting on lessons. The researchers collected data via multiple videorecordings of teacher praxis (pre-, during-, and post-intervention) and analyzed using mixed-methods coding. Results showed two teachers making modest shifts in teaching with one teacher sustaining the changes after the study. The two other teachers made no shift. Chauraya and Brodie noted that well-structured learning activities can result in transformative change as shown by the first two teachers, while citing the lack of time, both for attempting to include all the learning activities as well as constraining lengthy, deep reflective dialogue may be detrimental to some teachers more than others. Because the latter two teachers were novices with significantly lower self-efficacy, the authors posited that some teachers might need more extensive engagement to influence their practice. Ultimately, however, Chauraya and Brodie concluded that PLCs are an important vehicle for teacher learning.

\section{PLCs have essential features and can provide opportunities for critical}

reflection. Although numerous researchers (Achinstein, 2002; Jones \& Thessin, 2015; 
Linder et al., 2012; Overstreet, 2017; Pancucci, 2008; Servage, 2008; Stein \& Imel, 2002; Stoll et al., 2006; Vangrieken et al., 2017; Westheimer, 2008; Wood, 2007a) alluded to the essential nature of critical reflection within theoretical PLCs to produce transformative learning, few researchers described the in situ processes that occur within PLCs and few describe processes that encourage critical reflection within the PLC. My intention was to address the literature gap with my study.

\section{Critique}

In this section, I offer a critique of the research literature pertinent to my study. I present a brief analysis of the literature regarding PLCs, reflection, and critical reflection.

PLCs. Recent literature extolled the benefits of successful PLC implementation for improving student outcomes (Graham, 2007; Linder et al., 2012; Maloney \& Konza, 2011; Overstreet, 2017; Pancucci, 2008; Riveros et al., 2012; Servage, 2008; Vangrieken et al., 2017; Vescio et al., 2015). However, the paucity of rigorous studies linking learning communities with actual changes in teacher and student outcomes is a significant gap (McComish \& Parsons, 2013; Ronfeldt, Owens Farmer, McQueen, \& Grissom, 2015; Vescio et al., 2008). Owen (2014) posited that although the literature indicated general agreement on the important characteristics of successful PLCs, which of the factors the researchers emphasized changed dramatically and could account for the differential degrees of impact PLCs had on teacher and student learning and outcomes (Owen, 2014; Vescio et al., 2008).

Another omission in the extensive body of research around PLCs is the insufficiency of studies describing the actual processes that occur within the school PLC 
as opposed to theoretical research on what should occur (Maloney \& Konza, 2011; Ronfeldt et al., 2015). The process within a PLC that I focused on specifically is critical reflection. While many studies alluded to the importance of constructive collaboration, community, inquiry, and difficult conversations that lead to transformative outcomes, very few explicitly named critical reflection as a requirement for productive PLCs (Servage, 2008). My study contributes to the body of literature regarding the reflective processes within PLC and teacher insights into (a) the role of critical reflection on teacher learning and praxis and (b) the methods to improve the PLC process.

\section{Critical Reflection}

Teachers who regularly engage in reflection are more effective (Dewey, 1910; Laverick, 2017; Mezirow, 2000; Šarić \& Šteh, 2017; Schön, 1983). I previously delineated the important link between teacher effectiveness and improved student outcomes (Bakkenes et al., 2010; Cochran-Smith \& Lytle, 2009; Darling-Hammond \& Sykes, 1999; Durden \& Truscott, 2013; Laverick, 2017; Šarić \& Šteh, 2017). Culturally responsive teaching, a practice that my and many other districts have been working to attain, has critical reflection at its roots (Durden \& Truscott, 2013; Gay, 2002; Siwatu, 2006). Addressing the persistent gap in measures of achievement between the nondominant cultures and the dominant cultures is the most common PLC directive we hear in my district.

In their 2013 study, Durden and Truscott addressed the incongruity of working toward academic equity with culturally relevant pedagogy without critically reflecting and examining the social, cultural, economic, and political contexts. The investigators 
surveyed twenty-two elementary preservice teachers (PSTs) teachers using a 48-item cultural relevancy questionnaire. From this pool, they invited six participants to participate in the focus group for deeper study, based on ages and their wide range in viewpoints. One researcher was a participant-observer in group interviews, reflective blogs, and observations. Using case study methods, Durden and Truscott examined the reflective habits of three of the six PSTs over 10 months as they navigated course work and practicum in urban settings. Durden and Truscott reported two main findings: (a) Although the three teachers concentrated their reflections at the micro-level (classroom and self), they were able to extend their knowledge beyond their classroom and their roles in society, and (b) Critical reflection did not necessarily produce culturally relevant practices. Critical connections of how the context impacts the students is necessary. Teachers need an understanding of the culturally relevant ideology and the knowledge of how to implement the practices and why they are implementing certain practices. Durden and Truscott concluded that the tenets of culturally relevant practices must guide the critical reflection.

As I noted previously, literature mentioning the importance of critical reflection in PLCs is abundant, but studies rarely, explicitly link the act of critical reflection (and the communal discourse which ensues) with the hoped-for transformative learning in PLCs (Brookfield, 2000; Šarić \& Šteh, 2017; Schön, 1983; Servage, 2008). Further, Šarić and Šteh (2017) posited that a large discrepancy remains between "the professed goals and the actual reflective practices of teachers" (p. 67). In other words, without intentional critical reflection, the promise of transformative change would be unlikely, if not 
impossible. I agree with Šarić and Šteh's position and assert that my study of intentional critical reflection within an equity-focused PLC could offer a nuanced link between critical reflection and teacher transformative learning.

\section{Review of Methodological Literature}

In this section, I outline the methodological design for my study. I selected a qualitative collective case study methodology to study how critical reflection unfolds in an equity-focused PLC. Yin (2009) described a case study as an empirical inquiry investigating a phenomenon in its context, in situ (Yin, 2009); others defined case study in terms of delineating the object of study (Merriam, 1998). Both definitions worked well for my study, because critical reflection has the potential to occur within a PLC. In my study, I delimit and bound the case to an existing equity-focused PLC.

Different research designs have implicit ontological worldviews (Guba \& Lincoln, 2005; Merriam, 1998). For example, the positivist paradigm lends itself perfectly with quantitative methodology and the hard sciences, because the ontological view posits that knowledge and reality exist externally and are quantifiable (Guba \& Lincoln, 2005; Kuhn, 1996). Kuhn (1996) acknowledged, however, that in certain fields, knowledge was not be so black and white. He wrote, "What a man sees depends upon what he looks at and also upon what his previous... experience has taught him to see" (p. 113). The postpositivist paradigm is useful for researchers who believe that a knowable reality exists and accommodates different viewpoints and definitions for that reality (Creswell, 2007; Guba \& Lincoln, 2005). Another worldview used to frame knowledge and reality is the interpretivist or constructivist, which lends itself nicely to my study. 
The constructivist paradigm views knowledge and reality as socially and culturally mediated through context—one co-constructed by the individual and the group (Creswell, 2007). Qualitative method allows the researcher to capture and understand the complexity of participants' views or meaning-making experiences (Creswell, 2007; Maxwell, 2005; Merriam, 1998). "Qualitative research is a situated activity that locates the observer in the world...consisting of a set of interpretive, material practices that make the world visible" (Denzin \& Lincoln, 2005, p. 3). By taking an "unseen" phenomenon, critical reflection, and the processes of a PLC in situ, and making them visible and understood through interpretive rich description and analysis, I assert that qualitative case design was appropriate for my study.

Using the qualitative case study approach, I could explore the complex process of how critical reflection unfolds within the situated, social construct of a PLC. I am interested in discovery, insight, and interpretation... in context, not hypothesis testing (Merriam, 1998, p. 29). The case study approach allows for the interpretation of the intricate interplay between theory, data, and teachers for making valuable interpretations and explaining the dialectic relationship that exists between the social structures, the professional learning communities, the human agency, and the will of the teachers to learn and change (Maxwell, 2005; Merriam, 1998; Yin, 2009). In the following paragraphs, I discuss similar case studies to justify my methodological selection.

In a mixed methods case study, Graham (2007) studied the relationship between PLC activities and teacher improvement of a small core of teachers at a middle school over a one-year period. Graham employed a pre-survey to assess which PLC activities 
teachers had participated in. Graham analyzed the activities that correlated with changes in teachers' behaviors and praxis with descriptive statistics. The researcher then conducted in-depth qualitative interviews and triangulating the data for consistency and validity. Results of the research indicated a strong, positive relationship existed between professional learning activities and teacher improvement, although the relationship proved complex and contingent upon multiple factors. Graham concluded with a viable model to encourage PLC activities that are more likely to result in transformative teacher improvement, underscoring, however, that fidelity to the activities cannot promise improvement without thoughtful, substantial conversations and trust between the members. Graham asserted teachers must learn to develop the skills and attitudes required to have open conversations and productive conflict. Intentional critical reflection prompts (and the time required to allow for deep process and dialogue within the community) are essential to sustainable and effective PLCs.

In similar study, Maloney and Konza (2011) conducted a case study at a primary school to examine the processes undertaken within a PLC that encouraged reflection and reflective practice. In this study, the researchers took on roles as participant-observers, attending all meetings and discussions, as well as facilitating the PLC activities. Maloney and Konza collected data using narrative recordings by participant-observers, participant interviews, focus groups, and a survey. Results were inconclusive as to whether the PLC provided significant support to nurture reflection and teacher learning. Some found the activities encouraged deeper reflection and learning. Others never adapted to collaboration, speaking out, reflective dialogue, or difficult conversations. 
Ultimately, Maloney and Konza concluded that more democratic agenda-setting fosters deeper individual commitment to both the PLC process and the shared culture. For this reason, I chose not to set the agenda or facilitate the conversation in my study to minimize feelings of power disparity. Again, like the previous study, trust and shared culture were vital to the PLC process.

A third study used qualitative case design to describe the micro-interactions a meaning making activities of dual-language and ELL teachers in a literacy-focused, virtual PLC (Steeg, 2016). Researchers collected data via interviews, videos of teacher practice, and transcripts of the PLC. Narrative, analytical vignettes indicated that videobased reflections acted as a stimulus for improved teacher collaboration, meaning making, and more reflection. The needs and interests of the teachers directed the topics and learning activities in this study. Steeg (2016) noted that participants shared a strong, trusting community by presenting several, transcribed interactions indicating a lack of judgement and hostility among the teachers. Steeg concluded by discussing the power of providing a space for communal, critical reflection that leads to increased teacher learning and how easily that knowledge transfers to the classroom. Like my research, Steeg did not claim generalizability, but rather offered a detailed account of one specific PLC.

Rather than the video and audio recordings and transcriptions Steeg (2016) employed case study to capture complex interactions, I decided to use field notes from my direct observation for gathering a sufficiently detailed encapsulation of the intricacies of PLC. This decision allowed me to compose a rich description of how critical 
reflection unfolded in PLC, as well as an accounting of other PLC processes (e.g., the conversational interplay of members negotiating understanding (Yin, 2009). My role as participant-observer afforded me with unique access to "the inside" information of the community (Yin, 2009). I incorporated "an active membership role" (p. 101) because I participated in the central activities of the PLC while observing (Merriam, 1998). More specifically, my role was a researcher-participant, "one who participates in a social situation but is...only partially involved” (p. 102). To reduce bias, I remained particularly alert to how my presence affected the activities and the participants and considered the effects during interpretation of the data (Merriam, 1998; Yin, 2009).

For my study, I opted to conduct semi-structured interviews of three teachers in the PLC. Merriam (1998) suggested that interviewing is necessary when the behaviors, beliefs, or feelings under study are not visible. Further, a semi-structured format for the interview was appropriate to ensure open-ended, conversational sessions with my participants, yet remain pointed toward the topic of my research inquiries (Merriam, 1998; Yin, 2009).

I also elected to use reflective writing prompts at the end of a PLC meeting to stimulate reflection. Having used reflection prompts extensively in my own classroom, I found it to be a low-risk way for students to provide immediate feedback and thoughts about the lesson's content. Within the study, I used samples of reflective writing similarly with my participants to provide a reflection prompt about the day's PLC topic or artifact examined. 
Last, I conducted pre- and post-surveys (i.e., Survey of Reflective Practice: A Tool for Assessing Development as a Reflective Practitioner Self Assessment) to ascertain teachers' self-reported pedagogical efficacy beliefs and attitudes toward PLCs for professional growth (i.e., learning) and their classroom practices.

\section{Summary of the Research Literature and Application to My Study}

In this chapter, I delineated my reasons for exploring the phenomenon of critical reflection within a PLC. I included a review of the conceptual and research literature with respect to PLCs as an important and potentially transforming context for teacher learning and continuous improvement. I further outlined a rationale for studying the role of critical reflection in PLCs, and specifically, its essential role in advancing transformative learning. Developing healthy, productive, and sustainable PLCs would require fostering responsibility for individual ideas, tolerance for the views of others, and a capacity to negotiate differences (Meier, 2000). The promise of PLCs, to be a nurturing forum for continuous improvement and transformative teacher learning, would remain elusive unless the participants could engage regularly in communal critical reflection. I concluded by offering literature support for my research design and methodology, as well as similar case studies from other researchers. 


\section{Chapter 3: Methods}

The purpose of my study was to explain and describe teachers' critical reflection in an equity-focused PLC within a suburban high school. As noted previously, PLCs are spaces where teachers may collaborate, reflect, and discuss various educational issues to improve practice and teach students (Dufour \& Eaker, 1998; Hord, 2004; Martin-Kniep, 2008; Servage, 2006; Westheimer, 2008). The problem of practice is that teachers have few opportunities to reflect critically within PLCs. Although reflection is one of the core tenets of PLCs, evidence suggests the shortchanging of reflection in real-life school learning communities (Achinstein, 2003; Darling-Hammond \& Sykes, 1999; Overstreet, 2017; Servage, 2007; Westheimer, 2008). Engaging in critical reflection can be transformative for teacher efficacy beliefs, learning, and practice (Brookfield, 1995; Deissler, 2008; Servage, 2008). As a researcher-participant, I observed the rhythm of the procedures, conversations, and culture of a PLC, focusing on occurrences of critical reflection. I add to the body of research about conditions that inhibit transformative growth as a result of participation in PLCs without the benefit of critical reflection.

I explored teacher beliefs, as self-reported, over the course of their participation in the PLC through surveys and interviews. It is my position that purposeful reflection opportunities within the PLC platform may encourage a will to learn, as well as deeper thinking, increasing positive teacher outcomes (Bakkenes et al., 2010; Deissler, 2008). This study highlighted conditions conducive to learning and growth within learning communities, as well as suggested areas for improvement, thereby strengthening PLCs, teachers' learning and promoting more consistently positive student outcomes. 
Using surveys and interviews, teachers shared their thoughts about if and how PLCs helped them learn and transform their beliefs. My field notes during PLC meetings recorded the manner of resolution regarding teacher bias, assumptions, and discord in their learning community. I wanted to learn how teachers perceived the PLC process and its usefulness about their practice and gathered illuminative data through semi-structured interviews. The study addressed the need for research focusing on the relationship between critical reflection, teacher beliefs, and teacher practice, while providing a detailed description of the complex phenomena of critical reflection and teacher learning within a PLC. The following research questions guided my work:

1. How does critical reflection occur within an equity-focused PLC without prompting?

2. In what ways does critical reflection influence teachers' beliefs?

3. How does critical reflection influence teachers' perceptions of their practice?

\section{Research Method: Qualitative Case Study}

I employed qualitative, collective case study research design to explore how critical reflection unfolded in an equity-focused PLC. Guba and Lincoln (2005) asserted that the paradigmatic choice of the researcher guides the methodology of the study undertaken. Because interpretivists value the fluidity of reality and the multiplicity of voices in shaping data selection and analysis, qualitative methodology is more amenable to the interpretive paradigm.

Maxwell (2005) identified five particular intellectual goals that make qualitative research the preferred method for a particular study: (a) understanding the meaning of a 
phenomenon as the participants understand it; (b) understanding the particular context within which the participants are acting; (c) identifying unanticipated phenomenon influences; (d) understanding the process by which the actions take place, rather than the outcomes; and (e) developing causal relationships between the actions and the context. Qualitative research is about understanding phenomena and its processes from the perspective of the players and the situated context as completely as possible to develop or deepen the understanding between the actions and the context.

I selected case study because this design accommodated an array of evidentiary measures and instruments for comparative data, including artifacts, documents, field notes, journals, and interviews (Yin, 2009). I surveyed and interviewed teachers within a PLC to elicit the meaning and usefulness of critical reflection as they understood it. Although there is much theoretical information as to what should occur in a PLC, there is very little research as to what actually goes on within a real-life PLC. Furthermore, a gap in the literature exists regarding the essential nature of critical reflection in PLCs. My goal was to add to the literature on PLCs, particularly those focused on teacher learning. Specifically, I selected a qualitative case study research design because unanticipated influences on critical reflection in situ might unfold in the PLC. The complexity of the phenomenon and the myriad ways humans might interact within a PLC meant the flexibility of case study design is essential. I collected rich descriptions of the waxing and waning of a real-life PLC in action and the conditions that bolstered or inhibited critical reflection. Ultimately, I sought to describe a link between intentional critical reflection in PLCs and increased learning outcomes for teachers, as self-reported. I used 
the research questions not only to delve deeply into each case, but also to examine the pertinent information across the cases. In this way, I could examine individual teacher's perceptions and beliefs about reflection within PLCs as well as gain a collective view using cross case analysis.

After defining my problem (i.e., teachers having few opportunities to critically reflect) and stating my purpose (i.e., to explain and describe teachers' critical reflection in an equity-focused PLC within a suburban high school), I developed my overarching research question- - How does critical reflection unfold in a PLC and how does it influence teacher learning and practice? Because I pursued explaining and describing how a specific phenomenon developed within the bounded context of a PLC, I used qualitative case study methodology.

I investigated a phenomenon (i.e., critical reflection) within its real-life context (i.e., the PLC) as teachers experience it. My study needed a research design with flexibility and openness to refinement and change, because critical reflection is complex and the variables that influence it are numerous. A qualitative, case study allowed me to develop a narrative to elucidate the inner world of a PLC, while examining the beliefs and actions of select teachers within the group.

Although I chose qualitative methodology in part due to my study's inherent unpredictability, there are many different types of interpretivistic design including phenomenological, ethnographic, grounded theory, critical theory, and case study (Maxwell, 2005). Again, what I explored during my study guided me toward the appropriate design. Employing case study, I navigated the situated, social construct of a 
PLC. A case study is distinct from the other designs by its intensive descriptions and analysis of a phenomenon, but also by its bounded system - in this case, the PLC (Merriam, 1998).

Yin (2009) defined case study as investigating "a contemporary phenomenon (i.e., critical reflection) within the real-life context (i.e., PLC), especially when the boundaries between phenomenon and context are not clearly evident” (p. 13). Qualitative case study allowed for the study of how people situated in context, time, and history have constructed meaning (Merriam, 1998). I explored how critical reflection, a complex phenomenon, unfolded in a PLC, as well as described the inner-workings of a PLC (i.e., the context within which the phenomenon reveals itself) (Maxwell, 2005; Merriam, 1998; Yin, 2009). I explored how teachers experienced critical reflection in an equity-focused PLC. It is the interplay between theory, data and participants which allows for the valuable interpretations capable of explaining the dialectic relationship existing between social structures (i.e., the professional learning communities) and human agency (the will and action of the teachers) (Wolcott, 2008).

My study included the five components appropriate for case study as outlined by Yin (2009) in his guidelines: research questions, propositions, units of analysis, proposition-data links, and criteria for interpretation of findings. The first three components are:

1. Research questions - I framed my questions as "how" questions because case study generally uses how and why questions. My research questions explored how critical reflection unfolded in a PLC and how it influenced teachers. 
2. Propositions - I used my proposition that communal critical reflection in PLC is crucial to sustain teacher transformative learning. Through examination of critical reflection and its impact on participants with a PLC, I sought to link its essential necessity within PLCs to promote learning, specifically transformative learning.

3. Units of analysis - I used individual teachers, purposefully selected, as the units of analysis. I selected three teachers from an existing equity PLC and treated them as multiple cases.

Later, in Chapter 4, I discuss Yin's (2009) the last two components (i.e., link the data to the proposition, criteria for the interpretation of the findings).

\section{Participants}

All participants were teachers from a mid-sized suburban high school who were already attending and committed to the existing, equity-focused PLC. The community members were self-selected and worked around pedagogical issues of student equity and cultural responsiveness, so I anticipated these teachers would be more likely to engage in spontaneous critical reflection and be open to a researcher-participant amongst them. After disclosing my research goals and obtaining consent from group members, I invited the PLC participants to complete a survey of efficacy beliefs, as well a survey gauging their current level of reflection. From the large group and using purposeful sampling, I selected three participants for the in-depth case studies. Purposive sampling enriched my bounded, collective case study (Creswell, 2007; Yin, 2009). Each key participant had from high to low teacher efficacy beliefs and moderate to high current reflective practice 
as indicated on the CRTSE and the SRP surveys. By selecting participants that had differences in efficacy and reflective practices, I focused more readily on if and how critical reflection influenced their praxis and beliefs. I conducted within case, rich descriptive analysis of the individual's experiences and subsequently, thematic cross case analyses (Creswell, 2007). Because efficacy beliefs correspond to one's willingness to change and level of engagement with new practices, I hoped to mitigate the appearance that any differences in teacher learning and reflection were due solely to personality differences regarding willingness to change and reflect.

\section{Setting}

North River High School (NRHS), a suburban school with approximately 1500 students. Its population has a $29 \%$ sector of students eligible for free or reduced-price lunch, 22\% students of color, and 4.6\% English language learners (ELL). The school has four sections or neighborhoods, each with Grades 9, 10,11, and 12. The students generally take three to four core classes and two to three electives, (choir, band, technology, expressive arts, and world languages etc.) during a seven-hour, even-odd day rotation. The school also offers an International Baccalaureate (IB), Dual Credit (with the college), and Dual Language (DL) diploma.

The guiding principle of the school is to maintain a safe, inclusive, and equitable environment for students. The teachers enjoy a collegial, friendly staff in a wellmaintained newer building (est. 1999), and personable administrators. Many of the teachers have worked at the same school since its inception, although the previous principal left to open the new high school and nearly one third of the NRHS staff left 
with him. In general, the staff seems to like the new principal.

The school is in a period of transition with many new teachers and support staff, as well as a rapidly changing demographic. When the new high school opened, the district restructured the neighborhood-school boundaries, and the NRHS student demographics shifted abruptly. The school sits in an upscale neighborhood and the population was overwhelmingly White and financially stable. Now it is adjusting to an influx of students of color, language learners, immigrants, and the economically disadvantaged. The district expects the trend of changing demographics to continue for the next decade. Fortunately, the principal works tirelessly to maximize opportunities and equity for all students. The school attempts to keep strong relations with the community and parent-volunteers, although it is struggling to better communicate and include Latino and immigrant families. Teachers are under the supervision of the administrators, but primarily for procedural duties and goal setting. The administratorsvia district directives - mandates teacher attendance of one content-specific PLC, one cross-content group, and two free-choice PLCs each month.

The school board and subject-specific teacher-cadres determine the curriculum. A subgroup of core teachers from the district collaboratively established essential learning targets linked to the state assessments. National directives (No Child Left Behind Act (NCLB) and Every Student Succeeds Act (ESSA) mean that schools must have a School Improvement Plan (SIP) in place to track progress. NRHS' SIP states that students scoring at the proficient level on the state assessments need to increase $10 \%$ by the end of the 2017 school year. As noted previously, in comparison to district averages, 
NRHS has higher SES level and higher 2016 scores in both reading and math.

Population analysts expect these figures to change precipitously as the repercussions of the boundary changes take effect. In line with district goals, the schools' school improvement plan (SIP) revolves around learning teams focused on equity, collaboration, and learning.

The district strategic plan developed with community involvement and support, identified teacher collaboration as the key strategy for ensuring teacher learning and individual student growth and instituted early-release Wednesdays. Beginning in 2017, students have a weekly 90-minute early release, allowing teachers to collaborate in PLCs and participate in professional development consistently. My research studied the PLC from the existing early-release Wednesday groups.

I selected an equity-focused PLC using a purposeful sampling tactic. The community members are self-selected and work around pedagogical issues of equity and cultural responsiveness. My assumption was that because the teachers self-selected into a PLC with a progressive agenda, they might be more likely to engage in spontaneous critical reflection and be open to a researcher-participant amongst them.

\section{Procedures}

I used a qualitative, collective case study method to explore and form holistic understanding of the processes, perceptions, and influence of critical reflection within the context of a PLC. After obtaining written consent, I surveyed an existing Wednesday, early-release group focused on social and academic equity for students, specifically focusing on their beliefs regarding culturally responsive teaching. As a participant- 
observer, I had valuable access to the equity-focused PLC, which met once a month. I attended all meetings and engaged in PLC activities and discussion groups. I divided the research into two parts:

Phase I. After securing informed consent from the PLC teachers (see Appendix A), I administered the Survey of Reflective Practice: A Tool for Assessing Development of a Reflective Practitioner (see Appendix B) to six teachers, measuring the teachers' current level of reflective engagement (Larrivee, 2008). Next, I conducted another survey, Critically Responsive Teacher Self Efficacy Survey (see Appendix C), to ascertain teachers' efficacy beliefs as they pertained to teaching, their expectation of a student's outcomes, and their confidence in affecting change. Siwatu (2007) developed the Critically Responsive Teacher Self Efficacy Survey based on theoretical and empirical research. Based on survey responses, I used purposeful selection to invite three teachers for in-depth interviews. During one semester, I took field notes of the processes of the PLC to capture the essence of critical conversations and reflections that occurred spontaneously in the large group.

Phase II. I assigned a pseudonym to each of the three selected participants and organized any subsequent data with the given pseudonym. I secured data in a passwordprotected personal laptop, a dual password-protected smartphone, and a physical file in a locked cabinet in my personal office to prevent unintentional identification. I conducted pre- and post-participation interviews with the key participants for more in-depth study. The interviews allowed for a more detailed picture of the teacher, elaborated on their efficacy beliefs, and their perceptions regarding the utility of PLCs in terms of altering 
teacher behaviors, their personal meaning of critical reflection, and their demographics (e.g., years teaching, subject matter). During this phase, I also took field notes during the equity PLC and other staff PLCs.

\section{Data Sources}

As noted previously, I used surveys, written reflections, field notes, and interviews to explore critical reflection in the equity-focused PLC and its impact on teacher practice.

Surveys. Initially, I used the Survey of Reflective Practice: A Tool for Assessing Development as a Reflective Practitioner (SRP) (Larrivee, 2008). The 53-item selfassessment instrument is divided into four categories: (a) pre-reflection-reactive, general interpretations without thoughtful connection, (b) surface reflection-general, tactical thoughts regarding practice based on experience, not research, (c) pedagogical reflection-specific, persistent thinking about teacher improvement and student learning based on experience and theory, and (d) critical reflection-continuous engagement in reflective inquiry and examination concerning teacher praxis, thinking processes and how assumptions, values, cultural, and societal conditions affect classroom practices. The SRP survey measured the participants' perceived level of current reflective practiceinfrequently, sometimes, or frequently - pre- and post- participation. I received permission to use the survey from Larrivee (2008) (see Appendix D). The survey took participants approximately 15 minutes to complete.

Further, I conducted The Culturally Responsive Teaching Self Efficacy Scale (CRTSE) (Siwatu, 2007) at the beginning of the semester to garner a baseline of their 
assumptions, beliefs, attitudes, and experience regarding PLCs, practice, student attributes and student outcomes. The 40-item Likert-type questionnaire elicited selfreported information from teachers regarding their efficacy to execute a broad range of practices associated with culturally responsive practices. I received permission to use the scale from Siwatu (2007) (see Appendix E). The CRTSE required 15 minutes to complete. Post-participation of both surveys at the end of the study provided data for comparison.

Written reflections. I provided prompts to the key participants to encourage reflection regarding their regular PLC meetings. I adapted Brookfield's work (1995) on critical incident protocols (CIP) as a technique that asks low risk, exploratory prompts (i.e., describe an incident from the past week that makes you realize how great it is to be a teacher) to draw out themes and conversations (see Appendix F). The written reflections took less than 10 minutes to complete. If opportunities for critical reflection did not unfold organically during the large group PLC, I provided the additional prompts to encourage critical reflection through the activation of memories, exploration of experience, and consideration of power and equity issues during the separate meetings

Occasionally, the administration or facilitators provided prompts to cultivate deeper conversations that allowed me to take field notes describing the interactions between teachers, group processes for navigating disparate views, and for negotiating consensus. I noted any differences in quantity and quality of critically reflective conversations when given prompts or when not. 
Interviews. I conducted two in-depth interviews with the participants. The interviews were approximately 45 minutes each. Interviews are elemental in almost all qualitative studies (Merriam, 1998) and one of the most important sources of information in case studies (Yin, 2009). A good interview in a case study is more like a "guided conversation" with a specific line of inquiry goal but with friendly, non-threatening, open-ended questions (Yin, 2009). I obtained permission to record the participants in the interview. During the first interview, I asked questions to garner information about teachers' understanding of the meaning and function a PLC, their experiences in PLCs, their understanding of reflection, how they engage in reflection, and their view of an ideal PLC meeting (see Appendix G).

During the final interview, I asked participants to reflect on their practice using the learning activities and outcomes delineated in Bakkenes et al. (2010) to frame their perceptions of teacher practice, learning. By employing the language Bakkenes et al. developed as a scaffold to operationalize teacher activities and outcomes, I explored teacher growth and change (see Appendix H).

I used data collected from the Survey of Reflective Practice: A Tool for Assessing Development as a Reflective Practitioner (Larrivee, 2008), The Culturally Responsive Teaching Self-Efficacy Scale (Siwatu, 2007), participants' written reflections, and the interviews to address my research questions (see Table 2).

Table 2

Methods Matrix

Research Questions Data Source 
1. How does critical reflection occur in a PLC without prompting?

2. In what ways does critical reflection influence teachers' efficacy beliefs?
- Field notes

- Survey of Reflective Practice: A Tool for Assessing Development as a Reflective Practitioner

- Initial interview

- The Culturally Responsive Teaching SelfEfficacy Scale

- Initial interview

- Written reflections

- Survey of Reflective Practice: A Tool for Assessing Development as a Reflective Practitioner

- Final interview

- Written reflections

\section{Guided Experiences}

Specifically, I explored teachers' learning activities, with the assumption that knowledge requires active construction. These included:

1. experimenting - trying out new strategies, lessons, or new methods of interacting with students;

2. considering or reflecting on one's practice - either self-initiated or by external feedback;

3. struggling not to revert to old methods - the active resistance during periods of challenging implementation of new understandings to not fall back on old patterns; and

4. getting, sharing, and reflecting on new ideas to implement (Bakkenes et al., 2010). 
Further, I examined teachers' perceptions of learning outcomes defined as changes in knowledge and beliefs, or teaching practices. These included:

1. changes in knowledge/beliefs — conscious awareness, confirmation of an existing idea, or new ideas;

2. intentions for practice - intention to try a new practice, intention to continue a new practice, and intention to continue current or old practice;

3. changes in practice (as self-reported as more permanent) - new practice or back to old practice, and

4. changes in emotions - positive emotions (e.g., pride, satisfaction, positive expectations), negative emotions (e.g., irritation, fear, doubt), and surpriseunexpected revelations, positive or negative (Bakkenes et al., 2010).

Using guided experiences, I uncovered how critical reflection influences learning to gain understanding of the transformative process (see Table 3).

Table 3

Indicators of Change in Teacher Language with Examples

Indicators of change in teacher language Examples

1. Statements regarding learning outcomes I have learned that...

2. Statements of intention I am sure I am going to do this the same way next time

3 Use of comparative and superlative degree I think about those things much more than I used to.

4. Use of verbs that denote change (to gain, to go back, to change...)

I gain a lot by using this method 
5. Use of adverbs signaling change (before, now, suddenly, never...)

I tend to see and do things differently now.

6. Utterances of spontaneous insight

Now I see!

7. Utterances indicating surprise, pride, uncertainty

I was very surprised the students liked it.

\section{Role of the Researcher}

I contend that teachers have the power to improve their practice through consistent participation in a PLC that regularly engages in critical reflection. Teachers should examine their practice critically and with regularity, questioning their bias, assumptions, and blind spots (Badia, 2017; Brookfield, 1995, 2000). From careful examination of the problems of praxis through an equity-focused lens, teachers may become more culturally responsive, providing a more fruitful and productive educational setting for all students.

That said, I address my bias immediately. I am a 20 -year practicing educator, a strong supporter of public schools, a member of the union, and a former and current participant of scores of PLCs during my tenure. Therefore, I positioned myself explicitly with my study participants as a teacher, advocate, and researcher. As a participant observer, I was aware of my bias and assumptions by logging self-reflections after meetings and being present in the moment as well. According to Creswell (2007), “...objectivity is a chimera: a mythical creature that never existed, save in the imaginations of those who believe that knowing can be separated from the knower" (p. 208). Complete objectivity is elusive; however, researcher self-reflection and journaling, 
member checks, and triangulation limited bias and strengthened validity (Creswell, 2007; Merriam, 1998).

As a participant-observer, I recorded detailed, rich descriptions of the PLC as processes and interactions occurred, which allowed me to develop deep understanding of the factors that contribute to, encourage, or hamper critical reflection occurring spontaneously. Adopting the position of an insider researcher mitigated the natural tendency for a group to put on "company behavior" in the presence of an outsider, because the culture-sharing group and their activities immerse the researcher (Creswell, 2007).

\section{Data Analysis}

Before analyzing the collected data, it is important to consider the four tests of quality related to case study research design: (a) construct validity, (b) internal validity, (c) external validity, and (d) reliability (Yin, 2009). While internal validity is important for causal or explanatory investigations, it is less applicable to explorative and descriptive research (Yin, 2009). Therefore, I address construct validity, external validity, and reliability within my explorative and descriptive case study.

To ensure construct validity, I used multiple sources of evidence, established a chain of response evidence, and provided participants access to my data to check for accuracy. I followed these steps: (a) surveyed the PLC group, (b) used semi-structured open-ended interviews with key participants, (c) wrote extensive field notes from behaviors, dialogues, and processes I observed in the meetings, and (d) collected samples of written reflections. 
I offered the first questionnaire, the Survey of Reflective Practice: A Tool for Assessing the Development of a Reflective Practitioner (SRP) to the large group to garner a baseline level and quality of their current reflective practice. I offered a second survey at the end of the first PLC meeting, the Critically Responsive Teacher Self Efficacy Scale (CRTSE), which provided me with data to allow for a general idea of a teacher's willingness and openness to the idea that teachers have the capacity to affect change in students' achievement and outcomes (Tschannen-Moran \& Hoy, 2001). Research confirms that teachers' level of efficacy belief in their potential to overcome the effect of a student's environment or a teacher's belief in their ability to reach any student regardless of challenges, is a strong indicator of student and teacher learning and outcomes (Bray-Clark \& Bates, 2003; Love \& Kruger, 2005; Siwatu, 2007, 2011; Tschannen- Moran \& Hoy, 2001). A strong correlation exists between positive teacher efficacy beliefs as a preliminary stance and a teacher's willingness to persevere in challenging situations, change, and positively engage in professional development (BrayClark \& Bates, 2003).

The results of the SRP and CRTSE given to the entire equity-focused PLC guided my invitation to three participants for deeper study. With maximum variation sampling in mind, I purposefully selected three teachers with various levels of efficacy beliefs and reflective practices, as well as broad demographics (Merriam, 1998). Meg was a 47-yearold, White female with 20 years of teaching experience; John was a 60-year-old White male, with 40 years of teaching; and Carmen was a 29-year-old Latina female in her 4th year of teaching. For their self-reported CRT and reflective practices instruments, Meg 
reported lower levels of reflective and culturally responsive practices, Carmen reported medium levels of both reflective practice and CRT practice, while John scored himself high in both reflective and CRT practices. All three attended all meetings during the research period.

After selecting Meg, John, and Carmen, I conducted two in-depth, audiotaped interviews with each teacher at the onset and the conclusion of the study (30 minutes each). During the period I conducted the interviews, I continued to attend the group equity PLC meetings, as well as the equity-book PLC meetings. I took careful field notes focusing on the processes and micro-interplay within and between the PLC and teachers within the 90 minutes of meeting time.

Typically, to determine whether the study would withstand external validity and generalize to the broader population would require statistical analysis (Yin, 2009). However, Yin asserted that case studies rely on analytical generalization, whereby the researcher generalizes a set of results to a broader theory. By using a collective case study, I relied on Yin's 'replication logic' (p. 37)" to strengthen my study. First, I selected three participants and treated them as distinct units of analysis. Then, I analyzed participants' responses to a set of similar circumstances that should produce similar results if my presumptive assertion (i.e., that critical reflection during PLC is essential for teacher transformative learning to occur) is correct (Yin, 2009). Next, I connected my cross-case analysis to the theories of situated learning and transformative learning. By developing strong questions, having a strong theoretical framework in place, reducing my 
own bias, and using Yin's (2009) case study protocol, I contend that my design has construct and external validity.

To analyze the survey responses, I employed manual calculations and Excel® to evaluate the level of reflection and self-efficacy for each participant. Then using participant samples of reflective writing, interview transcriptions, and field notes, I coded responses and interchanges with ATLAS.ti ${ }^{\circledR}$ software. I used an initial cycle of organizing and sorting, employing descriptive and in-vivo coding to separate the data into categories of topics, participant words or phrases, and emotions taken in response to situations or problems (Saldaña, 2009). Then, I continued refining the categories with a second cycle of pattern coding and matching, further synthesizing my data into more meaningful constructs or themes (Yin, 2009). Subsequently, I provided a detailed description and interpretation of each case and the themes developed within the case, followed by thematic pattern analysis across the cases (Creswell, 2007). Then, I offered recommendations regarding the use of practical critical reflection in the workplace - in this case the PLC. Through this descriptive qualitative analysis, I developed a valuable story line to view my study. 


\section{Chapter 4: Results}

The purpose of my study was to explain and describe teachers' critical reflection in an equity-focused PLC within a suburban high school. I was curious to learn if and how teachers used critical reflection. Further, I wanted to explore the importance of critical reflection on teacher's practice and beliefs. My goal was to explore a problem of practice regarding teachers' limited opportunities to reflect critically within PLCs to improve teacher practice. To guide my study, I posed three research questions:

1. How does critical reflection occur within an equity-focused PLC without prompting?

2. In what way does critical reflection influence teachers' beliefs?

3. How does critical reflection influence teachers' perceptions of their practice?

In this chapter, I describe the context of the study and the participants, offer detailed profiles of the three cases, summarize the collected data, and outline the methods of findings analysis and thematic interpretations. I present the results of my study in both data tables and narrative passages to illustrate my cases' individual experiences with PLCs and reflection. I end the chapter with a summary of the results and set the stage for the findings and conclusions in Chapter 5.

\section{Study Context}

The suburban high school in this study was in a period of transition. Within the previous five years, the student population had shifted from predominantly White and affluent to increasingly Latino and economically struggling. Four years ago, one-third of the teacher population relocated to the brand new (more affluent) high school, including 
the principal. The new principal made inclusivity, culturally responsive practices, and equity a priority.

To this end, the administration encouraged two teachers with prior experience and interest in critical race conversations to form and lead a PLC focused on issues of equity. The purpose of the group was to study educator systems for bias and inequity and to raise cultural and racial awareness among staff members through purposeful activities.

In this qualitative case study, I explored the phenomenon of critical reflection within the situated social construct of a PLC. I recruited participants from an existing equity-focused teacher group. During the spring semester, I attended all the equity-PLC meetings, as well as the equity book group that was an extension of the PLC group to collect field notes. Additionally, I adapted written prompts from Brookfield's (1995) critical incidence questionnaire to inspire reflection after the meetings and collected the writings.

\section{Study Population and Participants}

The equity-focused PLC was the context of my study and source of the initial study population, consisting of 11 high-school teachers, five males and six females with wide ranging ages, specialties, and teaching experience. Ten teachers were Caucasian, and one was Latina, which was representative of the school and district teacher ethnic makeup (approximately 90\% Caucasian and 10\% people of color). After obtaining permission from the school administrator and PLC leaders, I presented my study and its purpose to this PLC. This particular PLC focused on addressing equity issues affecting marginalized students within the academic structures of the school, as well as suggesting 
whole school professional development and providing information regarding the larger staff's unacknowledged biases and assumptions. Outside of my presence, the PLC group members anonymously voted to allow observation of the group, as well as recruitment of participants for deeper study (see Appendix I).

During the second semester of the school year, the equity-PLC met six times, once a month for approximately 90 minutes during the district mandated 'early-release Wednesday' time set aside for teacher PLCs. Eleven equity-PLC members attended the first meeting. Of the 11, six teachers gave permission for me to include the result of their pre- and post- survey in Phase I of the study. I summarized the demographic information about these six teachers in Table 3 .

Table 3

Phase 1 Participants' Demographics Table

\begin{tabular}{lccccc}
\hline Participant & Age & Gender & Race & Subject Area & Years Teaching \\
\hline 1 & 46 & F & W & Spanish & 19 \\
2 & & & & & \\
& 47 & M & W & History & 18 \\
3 & & & & & \\
& 60 & M & W & Biology & 36 \\
4 & & & & & 14
\end{tabular}




$\begin{array}{cccccc}5 & 45 & \text { F } & \text { W } & \text { Art } & 20 \\ 6 & 29 & \text { F } & \text { L } & \begin{array}{c}\text { Dual } \\ \text { Language/ } \\ \text { Physics }\end{array} & 4\end{array}$

Note. $\mathrm{L}=$ Latina; $\mathrm{W}=$ White.

\begin{abstract}
Analysis of Data
Throughout the six months of my data collection, I kept a researcher journal with field notes and personal reflections from the pertinent meetings (e.g., equity book study, equity-PLCs), interviews, and transcripts. As a researcher-participant during the study, I took extensive field notes after the equity-PLC meetings to capture my impressions of the meeting regarding teachers' critical reflections and to write down my reactions for later analysis. I also took field notes during each interview to document teachers' recollections of how critical reflection unfolded in the current PLC meetings or in previous meetings (RQ 1). To study how critical reflection influenced teachers' beliefs and perceptions (RQ $2 \& R Q 3$ ), I collected data from pre- and post-surveys, written reflections, and, initial and final semi-structured interviews for within case and cross-case analysis.
\end{abstract}

\title{
PLC Analysis
}

The PLC meetings were 90 minutes in duration. Although two veteran teachers co-led the groups, the meetings lacked structure. The meetings would generally start with one of the leaders asking the group about a recent situation that occurred within the school, a common reading, or perhaps a prompt provided by the administration. 
Members would comment and respond as they wanted. The co-leaders did not guide the conversations, so the group often tumbled along in a winding organic, unscripted path, culminating on a different subject. On at least one occasion, tensions mounted, group members raised their voices, and some shed tears; however, no productive intervention ensued. Some members rarely spoke, while other teachers spoke often. During the meetings I attended, I did not notice a call to reflect collectively (or individually), although the co-leaders gave exit tickets asking for ideas for upcoming meetings.

\section{Survey Analysis}

For the Survey of Reflective Practice: A Tool for Assessing the Development of a Reflective Practitioner, I tabulated the scores according to the scoring key suggested by the instruments' author (Larrivee, personal communication). In this 53-item survey, the teachers self-reported the frequency with which they engaged in various reflective activities from the options of infrequently, sometimes, and frequently $(0,1$, and 2 points respectively, for the first 22 items). For the remaining 31 items, the options were in reverse order. The teachers' survey scores ranged from 72 to 102.

Tallying the Culturally Responsive Teaching Self-Efficacy Scale was straightforward. Each of the 41 items asked the participant to score themselves on their perceived teacher efficacy beliefs and cultural competence in different classroom scenarios on a scale of 0 to 100 . Of the 4100 points possible, the six participants scored between 1661 and 3507. From the aggregated scores for the participants, I made decisions about the teachers to invite for interviews. 
After scoring the instruments (i.e., Survey of Reflective Practice: A Tool for Assessing the Development of a Reflective Practitioner, Culturally Responsive Teaching Self-Efficacy Scale), I began the process of selecting three teachers for the interviews. Initially, I intended to have three participants with relatively similar reflective scores while maintaining variation in race, gender, curriculum specialty, and experience. As it turned out, I invited the only person of color who agreed to participate in the future interviews. Of the remaining participants, three were White females and two White males with similar teaching experience, so I invited the female teacher with the lowest self-efficacy self-assessment and the male with the second highest score. All three, Meg, John, and Carmen, agreed to participate in the interviews. Meg self-reported the lowest score of reflective practice, John self-reported the highest level of engaging in reflective practices, and Carmen scored in the middle. I summarized the participants' aggregated scores in Table 4.

Table 4

Participant Scores from the Survey of Reflective Practice and CRT Self-Efficacy Scale

\begin{tabular}{|c|c|c|c|c|}
\hline Teacher & Survey of & Practice & CRT S & Scale \\
\hline $1 *$ & 72 & $68 \%$ & 1660 & $41 \%$ \\
\hline 2 & 92 & $87 \%$ & 3505 & $86 \%$ \\
\hline $3 *$ & 102 & $96 \%$ & 3250 & $79 \%$ \\
\hline 4 & 80 & $75 \%$ & 2670 & $65 \%$ \\
\hline 5 & 100 & $94 \%$ & 2555 & $62 \%$ \\
\hline
\end{tabular}


6*

79

$75 \%$

2060

$50 \%$

Note. *Participants selected for case analysis

\section{Interview Analysis}

When conducting the interviews, I recorded each interview with Transcribe $₫$, a software application available for smartphones. The application digitally preserved the audio of the interviews and transcribed the recording via artificial intelligence within minutes of receiving the file. Then, I transferred the text files to my personal Google drive. I listened to the recordings again while concomitantly inspecting the electronic file and correcting any errors. After printing the written transcripts, I gave each teacher a copy of their transcript to verify accuracy and to address any adjustments they wished to make. Once this member check was complete, I began the process of reading and rereading the transcripts, while making notes of my impressions in my journal and analyzing each line. Next, I transferred the text to the qualitative analysis software, ATLAS.ti ${ }^{\circledR}$, for organizational assistance in the first cycle of the descriptive coding process, whereby I assigned a code to the topic of a passage or paragraph. Descriptive coding was appropriate for summarizing words or short phrases from the topic of a passage (Saldaña, 2009). I also used in-vivo coding (i.e. selecting verbatim quotes) to capture the unique voice and language of the participants (Saldaña, 2009).

Using constant comparison analysis (Merriam, 1998), I assigned 70 initial codes to the data (see Appendix J). I culled the codes to 50 by combining those with similar meanings. My final descriptive and in-vivo codes included such codes as "expertise of all," "disengagement," "community," "CFG protocols," "quality reflection," and "rich 
discussions." Using my interview questions to guide me, I further refined my list by assigning a color to each code, and then sorting them into 11 major pattern codes. Subsequently, I re-analyzed the data in terms of the purpose of my study, that is, how teachers experience reflection in PLCs and my research questions

During the cross-case analysis, I clustered the major pattern codes into six subthemes: effective PLC, ineffective PLC, engaged teacher, disengaged teacher, persisting with new praxis, or reverting to old praxis. I ultimately identified three major themes (a) preference for CFGs model of PLC, (b) infrequent opportunity for reflection, and (c) benefits of communal reflection.

A recurrent theme among the teachers was (a) their preference for the CFG model of PLC, with its protocols and structure. The teachers used their prior CFG meetings as the standard for what a PLC looks like when done correctly. The sense of inadequately executed PLCs, namely without guidance, reflection, or focus, led to frustration because of the missed opportunities for richer discussions and learning. Each of the teachers discussed their desire to become better, specifically to reach the most marginalized students, yet often felt that, ultimately, their ill-equipped PLCs stymied transformational learning. Another prevalent theme was the infrequent opportunity for critical reflection within the PLCs. This paucity of reflective opportunities restricted systemic exploration of sensitive topics (i.e. White privilege, microaggressions, and racial inequities). The lack of reflection also meant that group members did not acknowledge the emotional toll of the TOC and the singular experiences of marginalization and otherness, because of being one person of color in a 90\% White-populated PLC and school. The final salient 
theme I identified was the benefit of communal reflection. The teachers emphatically conveyed the value of gathering with their colleagues, amongst a community of experts, and exchanging ideas, offering critiques of praxis in non-judgement, and collectively and publicly reflecting on ones' practice. The participants referred to communal reflection as instrumental in determining their persistence with new practices in the classroom. These three themes became the foundation of my assertions, which I discuss in detail in Chapter 5.

\section{Presentation of Results}

In this section, I present profiles of each of the three cases to capture the participants' unique thoughts about PLCs and reflection. I also describe their level of professional reflection, efficacy beliefs, and willingness to introduce change into their practice.

\section{Meg}

Meg is an energetic, 47-year old, White Spanish language teacher with 20 years of teaching experience. Having started as a student teacher at the school at its inception, she has only taught at NRHS. Meg grew up in a Midwest, middle-class family, but spent several years in Venezuela when her father transferred there for work. Meg was popular among students for her big heart and passion; she appeared nearly as young as her students in both body and spirit. Meg was very excited about the research project and spoke animatedly during both interviews.

\section{PLCs}


Meg spoke earnestly about the purpose of PLCs and about the importance of building a learning community in which teachers can learn from one another. She commented:

Teaching can be an isolating profession, so the learning teams really helped build the community... everybody brings their own expertise. An ideal PLC would be where you share your expertise and learn from each other...building community and being a support for each other.

Remembering a particularly effective PLC, Meg recalled: ...all of it was so empowering to [be a part of] ...we would discuss whatever happened, or observed, and interacted with all of those things...you go in to an observation of a classroom or a protocol with a problem of practice [brought by another teacher] with a lens, and you offered your observations to that teacher...but you have to have norms and you have to really trust your colleagues. When asked to elaborate on the differences between valuable PLC experiences and the ones that were not, she said she preferred "more structured, more task-oriented [PLCs], where you can check that box and feel like it was being productive." Well-developed PLCs are most effective, and include criteria like "teacher equality," "choice," "voice," "authentic dialog," and "reciprocity" of participation, when steeped in strong peer relationships to maintain ongoing, intensive learning (Overstreet, 2017).

\section{Reflection}

Meg showed a good deal of insight when discussing reflection in PLC — about what worked and what did not. She explained, “...I mean like okay, if you're going to 
reflect on something then what is the outcome? I need to have a task or a topic to focus on... a discussion or a product as a result of the reflection!" Meg also considered different ways of reflecting, when she mused:

...is reflection discussing and thinking about what worked in a lesson, what did not? Or where you can do better next time, like it is the next time that is important to me, a solution of sorts. But is it writing about it, an internal dialogue, or hearing other people talk and [participating] in a discussion? For me, discussing is the [reflective] process that is most effective... hearing and seeing through someone else's lens.

Meg reiterated how she needed focused reflection. She noted:

We were always talking about different strategies in our PLC, but it was anecdotal, not purposeful. We missed an important piece-because [although] we learned different strategies, and you had the choice to try one or not, but I did not have to report back to anybody about it, to reflect on the process.

She referred to communal reflection as a "communicative action," when she and her colleagues shared" their perspectives and discussed them. She conceded it might not be as important for everyone:

It has been interesting for me to team teach with Steve this year, for example. I think reflecting together is much more effective for me than for him. It is been interesting to see. I really spend a ton of time processing and he can just be like...with really strong convictions and not over process. 
PLCs without guided prompts to encourage more holistic thought, can stunt collaborative dialogue and decision analysis, and therefore, transformational learning (Sims \& Penny, 2014).

\section{Changing Practice and Shifting Efficacy Beliefs}

Having a student teacher turned out to be very impactful for Meg, as she had the opportunity to reflect with him. As she shared her practice with him, she saw her practice through his eyes and found it "such a healthy way to look at your own practice.

For the first time in the interview, Meg showed exasperation over the many missed opportunities in her PLC experiences_-ones that could have been agents of change in one's praxis, but usually were not. She lamented:

...that is part of your job, right? Like you are supposed to help that unmotivated student. So, then I wonder if I could try that... what if I tried this. [But] to ask and to reflect on your own, to try to do something differently, it really was not an effective strategy for me the past year and I was constantly frustrated by it.

However, she was also clear about what would have worked, stating:

I tried a lot of different things and for some students it just was not effective. I think one of the things that would be really helpful is sitting around with other teachers and saying, okay, well what do you think about this? And, you know, they could share what they have tried or suggestions. That would be so helpful. Meg thought CFGs were a much better iteration of the generic PLCs for their effectiveness and potential to change one's beliefs and praxis. She reflected: 
The CFG was a much longer meeting and we had time to just sit there and quietly reflect...but if the time is not carved out for you, when would we do that? You know, it is like taking a bubble bath and contemplating or writing in your journal. It has to be more, and it has to be part of the agenda.

Meg wanted the trust and security of the structured CFG that had norms and protocols; she speculated, "If we had a systematic way to sort of process these things, more directed, it would be more helpful." She was not happy with the format of early-release Wednesday PLCs, bemoaning:

And that's what I was hoping those Wednesdays were going to be, but it totally did not work. So, I think the admin is trying, with the staff small group meetings like the equity book group, to have like a mini CFGs... but you can't have a mini CFG, first of all, it takes time...it's good to know your colleagues, you know, like you feel a little more willing to have each other's back and to hear what they're saying, to understand their perspective of the school. Because you are connecting with something on a deeper level.

Meg earnestly wanted to improve and deliver instruction that is more effective to her students but was losing patience with the administration's clumsy attempts at building learning communities. Like the other teachers, she felt that a teacher's time was too precious to squander on pointless, unproductive meetings.

\section{John}

John is a White, 60-year-old, veteran teacher with 40 years of teaching experience. He spent 20 years teaching in inner city Los Angeles before moving to 
NRHS, where he has taught Biology and Anatomy for the last 20 years. John grew up in an upper middle-class family in the same suburb as NRHS, where he attended a prominent Catholic private high school. As a teacher at NRHS, he senses that the students like him for his boisterous stories, his upbeat nature, and his ability to speak Spanish. John, a lover of the scientific method, embraced being a primary participant in a research study with gusto, though his responses were succinct.

\section{PLCs}

Relating how important PLCs are to one's practice, he recollected one of his most engaging experiences:

I had a really good CFG a long time ago, maybe 17 years ago, here at NRHS.

Our facilitator was really good [with] the Socratic Method and guiding the dialogue...by using those [protocols] so everyone could speak. One activity I really liked was where we exchanged student work and looked at that and then came back together and discussed...we would follow up [in the following] meeting...which is really different than what we do now in my PLC...it is all about [content] and... in the CFG it was about improving practice, which I like better. The CFGs were structured...in a good way...everyone participated...[whereas] in the regular PLCs now, you can just sit there...not even engaged."

Because the expectation of participation is not part of the structure of the PLC, unlike the CFG, the benefits of feedback and idea reciprocity are mitigated (Overstreet, 2017). 
On a final interesting note, John indicated he was most engaged in PLCs "when people of color and LGBTQ spoke about their experiences" and least engaged when the discussions were not applicable to the science classroom. Notably, Carmen said she often feels like the "entertainment or an experimental specimen for the White teachers" at the meetings. This highlights the disparate experiences between teachers of color and their White counterparts (Brazas \& McGeehan, 2020).

\section{Reflection}

John believed reflection to be a necessity and a natural part of the teaching process. He reported:

Reflecting on practice is an everyday occurrence for most good teachers...How could I have done that lesson better? What can I tweak to make it better? How can I help Jimmy understand? You constantly reflect on your practice...but PLCs and CFGs are just a more structured way to do it.

John felt he was "personally motivated to look for bias..." He shared, "I'm internally asking and then internally evaluating, particularly with the equity group that forces me to look even more at assumptions and bias. He felt that "teachers reflected more than any other profession, except maybe doctors." Although John believed all teachers reflected as a natural part of their job, research indicates that cursory reflection, or simply reviewing the previous actions taken, is insufficient. Effective teachers must reflect and search for internal consistencies (and inconsistencies) between their beliefs and their actions, preferably within a reflective inquiry structure (Wlodarsky, 2005). Importantly, the reflection should occur within a collaborative inquiry structure, such as a PLC, to 
reduce individual bias and provide support in aiding the progression of critical analysis of behaviors, thoughts, and actions (Laverick, 2017).

\section{Changing Practice and Shifting Efficacy Beliefs}

When asked if and how his practice had changed because of his participation in PLCs, John had a lot to say. He stated:

I really thought the CFG- type PLC changed my practice because I was with veteran teachers and I was already a veteran teacher myself...but I felt like they gave me a perspective that was really good, really academically oriented, very Socratic...I just really respected my colleagues and their opinions...they were friendly about it, but they told me where they thought I could improve and that was great! I became better at questioning, and using Socratic Method and I paid more attention to the dynamics in the classroom...

John reported, "I think the equity team meetings help me... to realize my White privilege and the fact that students come from such different backgrounds from the background I came from." He noted that he needed to "figure out which students need more scaffolding." He added, "So I need to learn how to relate to the different group - ethnic groups — of my students." John displayed a more nuanced level of self-awareness between the initial and final interviews and perhaps, this explains the subtle negative shift of his scores measuring self-reflection and self-efficacy. As he became more aware, John might have recognized some areas of self-inflation regarding cultural responsiveness. Although Meg and Carmen thought the critical reflection in the equity-PLC was minimal, 
John — a neophyte on the journey of examining his own privilege - found it stimulating and transformative.

\section{Carmen}

Carmen is a new teacher with four years of teaching experience, three of them at NRHS. She is a 29-year-old first generation Mexican who grew up in Hawaii, where her immediate, working-class family lived. Carmen teaches Dual Language Physics, Engineering, and Robotics Design, as well as AVID $^{\mathrm{TM}}$ classes. AVID ${ }^{\mathrm{TM}}$, an acronym for Advancement Via Individual Determination, is a national "untracking" program designed to help underachieving students with high academic potential prepare for entrance to colleges (https://www.avid.org/). Her primary responsibility is the freshmen physics education of roughly 170 students, 50 of whom she teaches in Spanish. Recently the state recently named Carmen "High School Science Teacher of the Year" for the region. Her colleagues and students regularly look to her for her academic knowledge as much as her innovative and culturally responsive teaching techniques.

\section{PLCs}

In general, Carmen did not feel engaged with the equity PLC. As one of three teachers of color in a school of more than 80 teachers, she often felt her race was a barrier in the PLC. She recalled, "So many people were at the beginning of their social equity journey..." and more often than not the conversation would shift to whether [equity] should even be a topic of discussion “...it was painfully obvious that they were totally clueless as to the problems that [I] felt existed." At one meeting, after a teacher shared that several students reported a teacher grading inequitably, the question of whether it 
was fair to "assassinate" the integrity of said teacher without all the facts consumed most of the time. Other teachers regarded the mere questioning of a teacher's responsiveness defensively and it elicited visceral responses peppered with violent terminology. In other words, the teachers did not have a thoughtful examination of how "the staff as a whole" could be contributing to the problem.

Although Carmen thought the equity PLCs “weren’t super beneficial or productive" she disclosed that she actively "sought out the professional communities of the English Language Development (ELD) department" for their thoughtful and inclusive processes. She appreciated the trained, balanced facilitator who guided the group and liked the organization of the meetings. The teacher-leader stimulated dauntless discussions, prompted deep thought, and extracted the voice of everyone. When comparing those meetings to NRHS PLCs, Carmen expressed disappointment. She felt the absence of a trained, impartial facilitator was unconscionable, saying:

I wish the organizers had put more thought behind the meetings. What I wish would have happened was just some preparation...groups were led by teachers who themselves did not know what they were doing. They were not very strong leaders in this context, right? So how could they be there, expected to be taking on this role? Like it was very unfair to ask them and us.

Without a trained facilitator to lead the group, whenever a sensitive subject arose, as one of the few people of color in the equity PLC, Carmen felt like the token POC:

At some point [the other teachers] kind of started looking to the people of color for explanations. As though we could speak on behalf of, like what, all the 
minorities that are represented in this school! It takes energy and effort; it takes an emotional toll to be involved in those kinds of interactions as a person of color. Sometimes you are personally attacked or having to defend yourself or having to call out other people for their insensitive comments or actions. Often that is when someone a white person of privilege, is either extremely offended or even starts crying. Which is very insulting, because it is, like, let me have this moment. Why does it have to be about you?

Not only was there no professional, trained facilitator to guide these important conversations, the meetings were shapeless and unfocused. She lamented:

The purpose of the meetings and of the group were never well defined or fleshed out, which for it to be effective will have to be defined at some point. It seemed like we were supposed to direct where the whole staff and school was going to go in terms of creating an equitable culture and, at some point, I felt like we were even going to take on the district when the district or the system was not working, but the plans and actions never got off the ground. I think it was just pretty unstructured.

As Carmen described her experiences with the equity PLC, her disenchantment was evident on her face and even her shoulders slumped as she spoke about her encounters within the PLC. "No norms had even been set," she continued, "and to have to talk about very intimate, intense political beliefs. It is uncomfortable and I recognize it first thing when I walk into one of these groups. It's not safe.” Carmen's experiences are not unique. Educators of color are regularly asked to manage their colleague's White 
fragility, while taking on the antiracism in the schools and district (Brazas \& McGheeha, 2020).

\section{Reflection}

Carmen was quick to point out that the equity PLC meetings were not well structured. On one occasion, two members served as de facto facilitators guiding the conversation, but because there was no structure, the meeting discussions meandered without focus, and seemed hostile at times. She recounted:

I mean, I think just from the get-go, everyone was coming to this meeting cold and almost on edge, because we knew that we were about to talk about some things that we were not prepared for. There was no preparation involved...not even a prior training.... even just to navigate our own identities as a starting point.... we are all kind of strangers.

She pointed out that the fault did not lie with the teacher-leaders, who facilitated the PLCs. The administration did not provide the trainings necessary to navigate sensitive issues.

Little reflection took place in the meetings and conversations often became contentious. Carmen recalled one particularly heated exchange, saying:

We had been given an article about culturally responsive teaching, a very basic, like introductory techniques...we were given time to read it. One teacher quoted an important line from the article... and another teacher commented, 'Where do we draw the line? Where do we draw the line with [this] culturally responsive? I have so many students, so many Latino students' - she actually identified them by 
race - 'who never come to class.' She went on for a bit more, until I finally said, 'I think we need to slow down here... it is dangerous if we're making a connection between absenteeism and somebody's culture. In fact, that would be racist.' But the meeting [degenerated quickly] after that.

She found no reflection about implicit bias, no system for engaging in deep conversation, or no process for examining how to look within oneself and address assumptions. She continued:

These are the instances that just confirm that I am not a part of whatever journey it is that these teachers are a part of, like it is not that I am better than, it is just that my experiences are just too different...it was detrimental to my mental health and I quit going.

Regrettably, the district leadership, while committed to improving the numbers of teachers of color, has not been able to address adequately the needs of teachers of color, according to Carmen. Because of the daily slights and microaggressions she experienced - whether intentional or not - this talented teacher often entertained the idea of leaving the profession.

\section{Changing Practice and Shifting Efficacy Beliefs}

Carmen's whole demeanor changed as she spoke enthusiastically of the valuable skills she learned with her preferred PLCs from the ELD and Physics departments. She described:

I learned all the time at those! I would bring a technique or an idea—like my interactive notebook to engage more students - to the physics department PLC, 
and the team was so willing to partner with me and try new things. One of the teachers, whom I partner up with the most, is particularly helpful. We would take it [a new practice] to our classes and we would meet afterwards and reflect and discuss what worked, what did not, what we could adjust. And then we would go back and try it again. And sometimes, if it [the idea or skill] proved really great, we would take it to the larger group — even the district science meetings!

When I asked Carmen if she recollected a time when she struggled not to revert to old practices or patterns, she laughed and said, "All the time! The biggest practice change [for me] is really just shutting up...letting the kids process, that's a challenge!”

Carmen always kept her eyes and ears open for new ideas to implement in the classroom. Serendipitously, Carmen — on her way to work one day - heard an NPR piece about a teacher who would only speak to students using positive speak. She decided immediately to share the idea with her physics PLC, and they began experimenting and incorporating it within their practices. She has since added "positive speak" to her daily repertoire because not only was it so effective with the students, but it was also empowering for her.

\section{Interpretation of Results}

I designed the study to garner information about how reflection presents in PLCs and how teachers perceive reflection to influence their teacher beliefs and praxis. Further, the results may contribute to the body of knowledge surrounding PLCs, reflection, teacher efficacy, and teacher practice. Using a collective case study model, I collected comprehensive data to support my descriptions of how teachers reflect and to 
what end, how teachers experience PLCs, and what teachers believe to be the purpose and benefit of reflection and PLCs in terms of its' effect on praxis. I initially analyzed the data for within case patterns; then, I conducted cross-case analysis of the data to look for sub-themes through alignment with my research questions (see Table 5).

\section{Table 5}

Within Case Patterns and Cross-Case Sub-themes

Within Case Patterns

Cross-Case Sub-Themes

\section{Meg}

- Need for structure in PLC

- Need for accountability for teachers

- CFG preference

- Importance of communal reflection

- Frustration at missed opportunities

- $\quad$ Trying new practice and reverting

John

- Frustration with irrelevant content in PLC

- CFG preference

- Importance of communal reflection

- $\quad$ Trying and reverting

\section{Carmen}

- Effective PLC with reflection

- Ineffective PLC with infrequent reflection

- (CFG Preference over regular PLC)

- Engaged and learning

- Unengaged: (Frustration with poor PLC set-up: missed opportunities as an agent of praxis change; Emotional toll for TOC)

- Frustration at lack of deep guided conversations

- Confirmation of otherness

- Trained facilitator lacking

- Need for structured meetings

- Emotional toll

- Successful implementation of new techniques

- Persisting with or trying new practices vs.

- Reverting to old practices or not learning 


\section{Cross-Case Analysis}

In this section, I address the research questions and denote any themes that developed from cross-case analysis of the three teachers' surveys or interviews. I describe any similarities and differences between the cases. I identified six sub-themes from the cross-case analysis: (a) effective PLCs with included reflection vs (b) ineffective PLCs with infrequent opportunity for reflection, (c) teachers experienced a growth mindset or (d) disengagement and shutdown, and (e) persisting with new praxis was dependent on the amount of communal reflection and feedback the teachers had access to or (f) abandoning the new practice.

\section{Research Question 1. How does critical reflection occur within an equity-} focused PLC without prompting? In the interviews, the teachers generally reported few occurrences of reflection taking place in any of the PLCs they attended. This corresponded with what I observed when sitting in on the many meetings during the study. Carmen said, "We didn't reflect enough," and Meg commented, “I don't know that we were reflecting in a productive way," even when the opportunity clearly presented itself. Carmen described a situation when a racially charged accusation by a student surfaced during the equity PLC and there was no call to reflect or process the information. As Carmen said, "We should be talking about how to engage the staff in quality introspection and discussion of deconstructing personal bias and prejudice and structural and personal bias." Neither Carmen nor Meg thought the PLC included enough reflection. 
In contrast, John thought that plenty of reflection occurred in the PLC, “...especially at the end of the meeting...sometimes mentally, we write about it, other times or say it orally to the [group]." However, he ended by saying, "[We\} needed more [reflection], like in CFGs." In fact, these three teachers thought it would be beneficial to have more reflection and referenced the superiority of CFGs as a comparison. Each of the teachers spoke about CFGs when recalling a PLC that was particularly useful in terms of personal engagement and being beneficial to their praxis. They cited the "protocols," "time for reflection," and "trained facilitators to guide" the reflection and the processes that made the CFG-model superior to the less structured PLC. Table 6 displays the cross case sub-themes and common codes distilled from the data.

Table 6

Sub-Themes and Common Codes of Critical Reflection within an Equity-focused PLC for Research Question 1

Sub-Themes Common Codes

Effective PLCs Have Reflection

Time needed for reflection

Structured

CFGs are more beneficial

Protocols

Reflection

Rich discussions

Relevant content

Trust

Ineffective PLCs Have Little Reflection

Trained facilitator needed Community needed Poorly structured Missed opportunities Frustration 


\section{Research Question 2. In what way does critical reflection influence}

teachers' beliefs? The teachers all reported some level of change in their praxis because of critical reflection that occurred in previous PLCs. The difference in their pre- and post-surveys of self-efficacy also reflected those changes (see Table 7). Meg presented with an $84 \%$ increase in her efficacy, John showed a $1 \%$ increase, and Carmen indicated a $24 \%$ increase in efficacy.

Table 7

Changes in Teachers' Scores on the Critically Responsive Teacher Efficacy Survey.

\begin{tabular}{l|llll}
\hline Teachers & Pre-scores & Post-scores & Change in score & Percent change \\
\hline Meg & 1660 & 3055 & 1395 & $84 \%$ increase \\
John & 3250 & 3279 & 29 & $1 \%$ increase \\
Carmen & 2060 & 2560 & 500 & $24 \%$ increase \\
\hline
\end{tabular}

Interestingly, two teachers discussed how if they had been held accountable for their reflection, participation, and engagement, it would have been much more effective at changing attitudes, beliefs, and praxis. When recalling her experiences with CFGs, Meg reported that because of the CFG structure, if a new idea did not work out, "I'm going to bring it to the next meeting for discussion and processing." Carmen described at least five new strategies she incorporated in her daily routines based on the inspiration and feedback she received in her physics PLC dyad with her teaching partner, "we would try these things out, we would reflect right after using the strategy" and tweak as needed. John related a strategy that he had attempted, except without the benefit of reflection either before or after the attempt. When it failed, he abandoned it immediately, “...but 
with the CFG I would have probably kept it. I really respected my colleagues and their opinions." He felt it was very important to have that non-judgmental feedback. Table 8 exhibits the sub-themes identified from cross-case analysis of the data.

Table 8

Sub-Themes and Codes of Critical Reflection within an Equity-focused PLC for Research Question 2

\begin{tabular}{|c|c|}
\hline Sub-themes & Common Codes \\
\hline Growth mindset (Engaged) & $\begin{array}{l}\text { Community of experts } \\
\text { Rich discussions } \\
\text { Empowering } \\
\text { Raising awareness }\end{array}$ \\
\hline $\begin{array}{l}\text { Shut down and Closed off } \\
\text { (Disengaged) }\end{array}$ & $\begin{array}{l}\text { Disengagement } \\
\text { Confirmation of Otherness } \\
\text { Microaggressions } \\
\text { Frustration } \\
\text { Unsafe environment } \\
\text { Irrelevant }\end{array}$ \\
\hline
\end{tabular}

\section{Research Question 3. How does critical reflection influence teachers'} perceptions of their practice? I relied on the data from the Survey of Reflective Practice: A Tool for Assessing Development as a Reflective Practitioner and the interviews to gain insight into the teachers' perceptions of their own practice. In Table 9, I provide an example of how learning activities linked to learning outcomes from the text fragments.

Table 9

An Example of Learning Activities Linked Qualitatively to Learning Outcomes 
Summarized text fragments

Like how come I can do a strategy in one class that will be successful, but if I do it for another it fails, and I want to figure out why...

I have a fun helicopter simulator to show seed dispersion, but it takes a lot of time, so I used a flip book, which the kids could do themselves and it was still fun.

In our PLC we were discussing that students were not turning in labs, we were discussing ideas...I wanted to try the digital interactive notebook, I tried it and liked it...now we all use it.

First, I describe the four levels of the survey (i.e., Survey of Reflective Practice: A Tool for Assessing Development as a Reflective Practitioner); then, I discuss the teachers' scores. The Level 1 pre-reflection questions (\#1-14) from the survey examined the frequency with which teachers perceive they are reacting without conscious considerations. The Level 2 surface reflection questions (\#15-26) explored teachers' reliance on what works, regardless of value. The Level 3 pedagogical reflection questions (\#27-39) from the survey gauged the frequency with which the teacher: "Engages in constructive criticism of one's own teaching," "Has genuine curiosity about the effectiveness of teaching practices, leading to experimentation and risk-taking," "Has a commitment to continuous learning and improved practice," and "Sees teaching practices as remaining open to further investigation." The Level 4 critical reflection 
questions (\#40-54) measured the frequency with which teachers perceived themselves engaged in actions such as, "Views practice within the broader sociological, cultural, historical, and political contexts," "Considers the ethical ramifications of classroom policies and practices," "Recognizes assumptions and premises underlying beliefs," and "Is an active inquirer, critiquing current conclusions and generating new hypothesis." Participants' scores changed from first administration of the survey to the second administration as displayed in Table 10. Meg exhibited growth at a rate of $24 \%$ in in engaging in praxis impacting reflection.

Table 10

Change in Scores on the Survey of Reflective Practice: A Tool for Assessing Development as a Reflective Practitioner Self-Assessment

\begin{tabular}{lrrcr}
\hline Teachers & Pre-score & Post-score & Change in score & Percent change \\
\hline Meg & 72 & 89 & 17 & $24 \%$ increase \\
John & 104 & 100 & -4 & $4 \%$ decrease \\
Carmen & 79 & 90 & 11 & $14 \%$ increase \\
\hline
\end{tabular}

For Level 1, pre-reflection, the level where one is reflecting on the spot while in the classroom, her score increased from 18 to 24 , indicating that she is significantly less likely to rely on rash judgements. For Level 2, surface reflection, her score rose from 11 to 16 , meaning she is less likely to have confidence in ungrounded practices. For Level 3, pedagogical reflection, Meg had the maximum pre-score, meaning she had a deep understanding of students' contributions in the classroom and a strong commitment to 
learning about her praxis, and she remained steady in the post-survey. Notably, for the Level 4, critical reflection, which revealed her frequency of engaging in deeper, more nuanced reflection — as in, questioning commonly-held beliefs_-decreased from 13 to 10 (see Table 11). This could be due what I term a "belief-system correction." Like when an unrealistically inflated stock market undergoes a disruption that causes a sudden downward "market correction" to a more realistic price point, I believe that when a person experiences an information disequilibrium that they might have a "belief-system correction."

Table 11

Meg's Scores by Levels of Reflection

\begin{tabular}{lcc}
\hline Levels of Reflection & Pre-survey score & Post-survey score \\
\hline 1 Pre-reflection & 18 & 24 \\
2 Surface reflection & 11 & 16 \\
3 Pedagogical reflection & 28 & 28 \\
4 Critical reflection & 13 & 10 \\
\hline
\end{tabular}

Unlike the other two teachers, John's survey results exhibited a decrease in total reflection activities. He retained his maximum score for Level 1, pre-reflection, at 28. For Level 2, surface reflection, he decreased from 21 to 17, indicating he was relying less on his preconceived notions. For Level 3. pedagogical reflection, he grew from the nearly maximized score of 26 to a perfect score of 28 . For Level 4, critical reflection, John again remained steady at 26 (see Table 12). 
Table 12

John's Scores by Level of Reflections

\begin{tabular}{lcc}
\hline Levels of Reflection & Pre-survey score & Post-survey score \\
\hline 1 Pre-reflection & 28 & 28 \\
2 Surface reflection & 21 & 17 \\
3 Pedagogical reflection & 26 & 28 \\
4 Critical reflection & 26 & 26 \\
\hline
\end{tabular}

Carmen had post-survey scores that signaled an increase of $14 \%$ in reflective activities that influence praxis, although her scores increased in all 4 stages. For Level 1, pre-reflection, Carmen's score rose from 23 to 26, therefore operating less frequently in survival mode. For Level 2, surface reflection, she increased from 14 to 20, implying less frequent reliance on theories with questionable foundations. For Level 3, pedagogical reflection, Carmen revealed growth from 19 to 25 . For Level 4, critical reflection, she had an increase from 21 to 23 (see Table 13). Carmen's growth could be attributed to her increased exposure to microaggressions in the workplace after her experiences in the equity-PLC.

Table 13

Carmen's Scores by Levels of Reflection.

\begin{tabular}{l|cc}
\hline Levels of Reflection & Pre-survey score & Post-survey score \\
\hline 1 Pre-reflection & 23 & 26 \\
2 Surface reflection & 14 & 20 \\
3 Pedagogical reflection & 19 & 25
\end{tabular}


All three teachers spoke about how critical reflection during CFGs changed their practice. In Table 14, I summarized the sub-themes identified from my cross-case analysis of the data.

Table 14

Sub-Themes and Codes of Critical Reflection within an Equity-focused PLC for Research Question 3

\begin{tabular}{l|l}
\hline Sub-themes & Common Codes \\
\hline Persisting with New Praxis & $\begin{array}{l}\text { Practice improved } \\
\text { Students responded } \\
\text { Rich discussions } \\
\text { Communal Reflection } \\
\text { Shared expertise }\end{array}$ \\
& $\begin{array}{l}\text { Teachers on automatic } \\
\text { Reflecting with self only } \\
\text { Easier }\end{array}$ \\
\hline
\end{tabular}

In this section, I addressed the research questions through within and cross- case analysis of the cases. For each teacher, I discussed the patterns manifested in their stories. and the three 6 sub-themes that emerged from cross-case analysis: (a) effective PLC with reflection or (b) ineffective PLC without reflection, (c) growth mindset (i.e. engaged) or (d) shut down (i.e. disengaged), and (e) persisting with new praxis or (f) reverting to old practice. In the next section, I address limitations of the study. 


\section{Limitations of the Study}

This qualitative, collective case study addressed how critical reflection (a) unfolded in an equity PLC, (b) influenced teacher efficacy, and (c) influenced teacher practice as self-reported. While I have described in detail the unique voices of teachers as they talked about their experiences and beliefs regarding reflection, PLCs, and their praxis, which augments the findings from the in-vivo PLC interactions and teacher selfefficacy beliefs, as well as teacher learning, there are several limitations to the study. First, while the study allowed for in-depth exploration of three teachers' thoughts and perceptions, the small sample size limits the applicability of the findings. Second, I employed purposeful selection rather than randomized from a larger population. The findings might not be representative of the school population in general. The site itself also lacked diversity, which limited the diversity possible in the study sample. However, the important issues of the disconnect and the emotional toll brought to light by the lone TOC in the study should be explored more deeply. Third, the study occurred in a single site, again limiting the potential for the findings to be transferable to the wider population. Fourth, the survey data were self-reported and the interviews relied on teacher recall making the data susceptible to human distortion. Although I triangulated multiple data sources to mitigate individuals' spin and bias, the perils of distortion still exist. To mediate these limitations, I bolstered validity by conducting a cross-case analysis. Additional longitudinal study at multiple sites, including long-term follow-up observations with the teachers, would more robustly corroborate the survey results and 
teacher recollections, thereby strengthening the methodology and improve the study's generalizability.

Additionally, a notable limitation is researcher bias. I am a teacher in the school and district studied, I am a member of the equity PLC, and I knew and worked with the participants, which may have clouded my insight and interpretation. By consistent journaling and reflecting to check my partiality and my assumptions, I hope to have alleviated much of the bias, while remaining cognizant that a hallmark of human nature is to pick up sticks one likes when walking through memory's garden.

\section{Summary of Results}

In this chapter I have presented the results of this collective case study. The participants, Meg, John, and Carmen, experienced PLCs, as well as the equity PLC, in unique ways, but they also shared common perspectives about key features to improve PLCs including (a) communal reflection, (b) rich discussions, (c) a strong purposeful community, (d) the use of protocols to focus the meetings and ensure equity of voice, (e) time carved out for reflection, and (f) robust inquiry. The teachers also shared feelings of frustration at poorly organized PLCs, as well as dissatisfaction from missed opportunities to safely discuss sensitive issues and promote transformational learning. These perspectives led to the most salient pattern - the teachers' preference for the CFG-model of PLC. From the cross-case analysis of the common patterns, I identified six subthemes:

1. Effective PLCs with reflection opportunities,

2. Ineffective PLCs without reflective opportunities, 
3. Engaged teachers with growth mindset,

4. Disengaged teachers that are shutdown,

5. Teachers persisting in new praxis, and

6. Teachers reverting to old praxis.

In Chapter 5, I link the findings from this study to assertions about the interplay between successful PLCs, critical reflection, and teacher efficacy that contribute to my responses to the research questions. 


\section{Chapter 5: Discussion}

The purpose of my study was to explain and describe teachers' critical reflection in a PLC as well as how critical reflection influenced their practice and their beliefs. Using collective case study analysis, I considered data from surveys, interviews, and written prompts of three teachers' participation in an equity PLC over the course of one semester. The following research questions guided my investigation:

1. How does critical reflection occur within an equity-focused PLC without prompting?

2. In what way does critical reflection influence teachers' beliefs?

3. How does critical reflection influence teachers' perceptions of their practice?

In Chapter 4, I described the distinct voices and experiences of the three participant-cases, and I explained my analysis of the data from the interviews, two surveys, and responses to the research questions. I organized the data using the ATLAS.ti ${ }^{\circledR}$ software with description and in vivo coding. From this analysis, I identified the six cross-case sub-themes: (a) effective PLC with reflection, (b) ineffective PLC without reflection, (c) engaged teachers, (d) disengaged teachers, (e) persistence in new praxis, and (f) transience in new praxis.

In this chapter, I present the major findings regarding the purpose of the study organized by research question with the three major findings isolated from the cross-case analysis. For each teacher, I also discuss the patterns manifested in their stories. I continue the chapter with assertions based on my interpretations, in addition to submitting potentialities for the use of findings for education and policy leaders. Then, I 
discuss implications of these finding to offer recommendations for administrators and policy makers for the implementation of productive PLCs, along with suggestions for future research.

\section{Major Findings Related to Research Questions}

In this section, I offer a summary of the major findings organized by research question and themes. I identify three major findings: (a) reflection occurs in PLCs sporadically and ineffectively; (b) teachers are either engaged or shut-down; and (c) communal reflection is beneficial and empowering.

\section{Major Findings Related to Research Question 1}

I identified one major finding related to RQ 1: How does critical reflection occur within an equity-focused PLC without prompting. I found that reflection in general, and critical reflection specifically, infrequently occurred without prompting. Meg and Carmen both reported that critical reflection rarely transpired in their equity-PLC. However, the other teacher, John, reported that reflection occurred occasionally and shared that the PLC "made me think" and that he "sometimes do[es] it mentally." Although, reviewing one's thoughts internally can be beneficial, Mezirow (1990) wrote that an obstacle to reflection is the individual's inability to put aside their own biases and assumptions when examining events, thoughts, and practices. While my interview questions sought to solicit information from the teachers' perceptions of their current PLCs, the teachers repeatedly brought up earlier times spent in CFGs for comparison. Each of the teachers I interviewed referred positively to their experiences with the CFGmodel of PLC because of the considerable amount of reflection built in to the model and 
subsequent insights garnered (see Table 15).

Table 15

Major Findings Related to Research Question 1

\begin{tabular}{lll}
\hline Major Finding & Sub-Themes & Cross-Case Commonalities \\
\hline Reflection happens & Effective PLCs with & Time needed for reflection \\
sntly and ineffectively & on & Structured \\
& & CFGs are more beneficial \\
& Protocols \\
& Reflection \\
& Rich discussions \\
& Relevant content \\
& Trust \\
& Trained facilitator needed \\
& Community needed \\
& Ineffective PLCs with & Poorly structured \\
znt Reflection & Missed opportunities \\
& Frustration \\
\hline
\end{tabular}

Further, the three teachers all referred to the need for facilitators to guide the process, that "otherwise wouldn't occur," as Meg said. The teachers cited frequently the need for trained facilitators as necessary to guide challenging discussions, to encourage deeper discussion, and to set up norms that keep the trust of the group.

The literature supports the finding that reflection occurs sporadically and ineffectively (Westheimer, 2008; Maloney \& Konza, 2011). Because the focus of PLCs is often on the technical means of teaching instead of focusing on what the larger societal context of the teaching means, externally-imposed time constraints curtail reflection and, ultimately, transformational change (Servage, 2008). The teachers all referred to the missed opportunities that occurred in their PLCs, how in their PLCs they would discuss topics "anecdotally" and not follow up with implementation results, idea revisions, or 
reframing.

The teachers believed that teacher reflection was a moral imperative, but acknowledged that in the day-to-day business of teaching, pushing aside the acts of reflecting on oneself and one's practice. As Meg said, "When would I reflect if the time wasn't set aside?" Further, the teachers reported that teaching is isolating, so they traditionally work in their own classrooms with little time to engage in collegial or structured conversations about practice. Having the opportunity to examine one's thoughts with colleagues is vital to testing bias and assumptions. PLCs that effectively support teacher reflection "make room for the particular experiences emerging from classrooms and demand an exploration of the social and cultural contexts which afford opportunities in the learning process," (Steeg, 2016, p. 125). Riveros, Newton, and Burgess (2011) argued that for PLCs to be effective they must engage "in deeper reflection about the nature of action and practice in schools," to affect teacher learning and agency. In addition, Long (2012) asserted that PLCs must "assist teachers to deepen their understanding" (p.148) of quality education and practices to facilitate teacher growth.

\section{Major Findings Related to Research Question 2}

I identified a second major finding related to RQ 2: In what ways does critical reflection influence teachers' beliefs and attitudes? Teachers were either engaged and in a growth mindset or disengaged and shutdown. The teachers called the PLCs profound when their engagement with critical reflection led to a change in their beliefs and attitudes. Meg, John, and Carmen specifically cited the CFG model as instrumental in 
transformational change and learning because of the space and time allocated to reflect deeply with colleagues about ideas and practices. John credited the "small group discussions," that led to more reflecting and discussing, "without any sort of judgement," and produced changes by "...giving me a perspective that was really valuable," (see Table 16). He further elaborated how his views on equity changed and that, "the meetings helped me realize my White privilege," and to view the world from the perspective of the different students.

Table 16

Major Findings Related to Research Question 2

\begin{tabular}{|c|c|c|}
\hline Major Finding & Sub-Themes & Codes \\
\hline \multirow{11}{*}{$\begin{array}{l}\text { Teachers are either engaged } \\
\text { and learning or shut down } \\
\text { and closed off }\end{array}$} & Growth mindset & Engagement \\
\hline & Engaged & Community of experts \\
\hline & & Rich discussions \\
\hline & & Empowering \\
\hline & & Raising awareness \\
\hline & Shut down and closed off & Disengagement \\
\hline & Disengaged & Confirmation of Otherness \\
\hline & & Microaggressions \\
\hline & & Frustration \\
\hline & & Unsafe environment \\
\hline & & Irrelevant \\
\hline
\end{tabular}

The teachers reported either feeling engaged and learning or frustrated and shut down. Often, Carmen experienced detachment in PLCs because of the lack of critical reflection, especially notable during sensitive race conversations. Thoughtful conversations and guided reflections allow for members to test biases within the group, as well as the potential to experience transformational learning, Carmen recounted that 
frequently within in the PLC "group members were simply reacting," and conversations within the PLC devolved into "personal attacks, hurt feelings, frustration, and misunderstandings." Instead of being an opportunity for her to grow, she disconnected and decided that "I was not a part of the journey these teachers are on...the meetings weren't helpful to me." All the three teachers expressed irritation with the many incidents when they perceived the PLCs to be neither productive nor beneficial to their practice with John calling it "... a complete waste of time." Meg related that the lack of critical reflection and discussion prevented what could have been, "really helpful, sitting around with other teachers...sharing suggestions to try." Poorly organized meetings with ineffectual conversations obstructed growth, learning, and teacher buy-in.

The finding that teachers are either engaged in the PLC process by learning, reflecting, and changing, or disengaged, shut-down, and frustrated is not surprising. Forming a sustainable culture of collaborative learning within PLCs has experienced limited success (Servage, 2008). Bridging diverse ideas requires careful and purposeful attention to navigating difficult conversations, best guided by experienced facilitators (Achinstein, 2002, Rusch, 2005). Transformative learning theory works constructively with learner's vulnerabilities in the face of challenging ideas. Working collaboratively and testing bias and assumptions in a setting of shared norms allows for sustainable, transformative learning (Servage, 2008).

Further, as Carmen alluded to, schools risk alienating teachers of color by not addressing the onerous burden of "representing every minority" on the backs of the teachers of color. Educators cannot "move to professional learning without focusing on 
culture" and culturally responsive practices within the PLC, not as a separate entity, "but at every level of education, including teacher professional learning (p.200)" (Overstreet, 2017). Durden and Truscott (2013) posited that critical reflection across systems of influence in schools can fosters understanding of culturally relevant ideology and the knowledge of how to implement the practices and why they should be implemented.

When the PLC is praxis-centered and, therefore, relevant the "platform allows for rigorous and authentic examination" of teacher practices, beliefs, and attitudes thereby providing for "deep learning," (Long, 2012, p.149). Critical open-ended dialog and inquiry in a learning setting uses dissent as a tool to build community and reflection, "liberating us from strategic blindness and defensiveness," (Servage, 2008 p.70). Awkard (2017) wrote that school leaders: ...must be courageous, willing to challenge deeply held beliefs by asking instructionally focused questions that push teachers to recognize their own personal biases, give an honest appraisal of their own effect on students, and consider new ways of teaching... though difficult and uncomfortable work...can move teachers to pursue priorities for improvement (p.56).

Perhaps teachers will have to be their own leaders. In other words, demanding the training to lead challenging discussions and the training to hear critical examinations on their practice.

\section{Major Findings Related to Research Question 3}

I identified the final major finding related to RQ 3: How does critical reflection influence teachers' perceptions of practice? The teachers ubiquitously embraced critical 
reflection in community as essential to persisting with new praxis and the lack of communal reflection as the foremost cause of dropping new practices. Discussing the positives, the negatives, and the ideas for improvement with colleagues changed how they approached and implemented the practice. For Carmen, when teachers found the time to be with colleagues discussing the lesson experiences, "made all the difference, otherwise, it just wasn't effective." The shared conversations were "empowering," John offered, and gave the teachers the confidence to persist, "to be encouraged and acknowledged by your colleagues was super affirming for me,” (see Table 17). Long (2012) noted, “Teachers need opportunities to learn from each other, to work collaboratively, where their practices are acknowledged and valued (p. 149).”

Table 17

Major Findings Related to Research Question 3

\begin{tabular}{lll}
\hline Major Finding & Sub-Themes & Codes \\
\hline $\begin{array}{l}\text { Communal reflection is } \\
\text { beneficial and } \\
\text { empowering }\end{array}$ & $\begin{array}{l}\text { Persisting with new } \\
\text { praxis }\end{array}$ & $\begin{array}{l}\text { Practice improved } \\
\text { Students responded } \\
\end{array}$ \\
& $\begin{array}{l}\text { Rich discussions } \\
\text { Communal Reflection } \\
\text { Shared expertise }\end{array}$ \\
& Reverting to old praxis & Teachers on automatic \\
& Reflecting with self only \\
\hline
\end{tabular}

The teachers all wanted to improve their practice, and as Meg said, "to do the right thing and reach even the most unmotivated student." However, the lack of meaningful changes generated by "reflecting on your own," discouraged her. Meg acknowledged the complexity of the problem, "for practical reasons, the [district] didn't 
want to pay for our meetings," and "it might just be the nature of the beast, there's no easy solution." Because of the "busy-ness" of a teachers' daily life, Meg referred to the teachers as being "on automatic" and, therefore, more prone to "let me plug up that hole," rather than persisting with a new practice. She concluded that if the administration does not offer the teachers' opportunities for communal reflection, with "structured, carved out time for [us], when would we reflect?"

Overstreet (2017) argued that professional learning should happen in the classroom, asserting that "evidence of positive change in the student learning outcomes is a prerequisite to significant change in teacher attitudes and beliefs (p. 210)." In a study discussing the value of having a critical friend in one's classroom, Tillema and OrlandBarak (2006) wrote that knowledge construction is situated in context and "largely determined by the boundaries within which professionals work and participate" (p. 594). According to Kim and Hannafin (2008), situated learning in education means that practicing teachers learn through repeated classroom teaching experiences and interactions with other teachers. Meg described the most powerful learning she experienced was having other teachers in her classroom observing and the reflections and discussions that followed later in their CFG. The difficulty is in maintaining that model within the day-to-day pressures of teachers' lives without explicit space created by the administration. Just as important as creating the space is guiding teachers through the reflective process with patience and consistency. Keeping the discussion on the link between practice, instruction, and the effect on students allows for transformative interactions that produce lasting changes (Awkard, 2017). 
Communal reflection is key to promoting persisting in implementing new practices. In shaping thoughtful, structured collaborative PLCs, the administration is showing "a way of supporting practitioners to find the resolve to engage with and question change and to be proactive when confronting difficulties and dilemmas, both within themselves and with the system," (Maloney \& Konza, 2011, p. 76). In fact, researchers suggested that participating in collaborative inquiry and reflection is more important to transformative learning outcomes than their underlying professional beliefs brought to that activity (Tillema \& Orland-Barak, 2006). Awkward (2017) asserted that not only is communal reflection vital, but it is also essential that "teachers see this as a collaborative effort in which they have meaningful opportunities to steer the discussion, relying on teacher-leaders to serve as critical friends, not as judges or evaluators" (p. 55). Notably, Meg, John, and Carmen all referenced the lack of judgement they felt from their CFG.

\section{Unexpected Findings}

I expected to find that critical reflection was the missing link to a productive PLC that hampers transformational change in teacher's practices and beliefs. Instead, I found that the teachers perceived communal reflection, their deep collaborative discussions with their colleagues, to be the essential condition that had to be present. I intentionally distinguished communal reflection from collaborative reflection. Collaborative reflection indicated a group was working toward and reflecting on a shared goal, but I interpreted the teachers' words differently. Through their interviews, the teachers revealed a longing for a deeper community, with profound trust, to commune with one another about their 
practice and to challenge their thinking. This, they believed, would lead to critical reflection and the subsequent transformational changes in their beliefs and practices. I did not need to classify the reflection as critical. According to Mezirow (2000) critical reflection should include an attempt to uncover and identify personal, hegemonic assumptions and bias. Brookfield (2000) added that "the individual must engage in some sort of power analysis" (p. 126) of an experience and become aware of the oppressive structures in a broader societal context to be critical reflection. But significantly, Rodgers (2002) added the condition of engaging in reflection in community. To be in community to me means to be in state of trust and openness, where one can be exposed safely, not just in a group haphazardly slung together. Meg, John, and Carmen believed enough in the collective good of their own and their colleagues' desire to improve that, given the right environment, they could and would rise to consider societal contexts of inequities for the good of their students. In my interviews with the teachers, however, they felt that human communion and connection would allow for reflection to progress naturally in that direction when guided by trained facilitatorteachers that provided time for critical, synergistic discourse and analysis, which includes critical reflection. The facilitator-teachers, selected from the learning community itself, need training in artfully navigating difficult conversations and guiding the members toward deeper reflection and new negotiated understandings.

\section{Situated in the Larger Context}

As noted in Chapter 2, social constructivism views individuals as inextricably bound with society, engaged in conversation about their experiences of the physical 
reality. In social constructivism, learning occurs by assimilating new information into an existing framework and language through which learners communicate their thinking about that learning (Cobb, 1994). In my study of an equity-focused PLC, individual learning happened in a social context and collective learning was the interactive process of enculturation within a community. It was the language among the teachers in the equity-focused PLC engaged in discussion with one another that was central to each individual teacher's learning (Ernest, 1994).

In this study of teachers in an equity-focused PLC, situated learning (Lave, 1996; Lave \& Wenger, 1991) occurred in situ, or in its natural setting. The teachers coconstructed knowledge through their participation in the community. The community a collection of education-knowers; the experts and the novice become more equal, all knowers around the subject (Fenwick, 2000). Activities in the equity-focused PLC were integral to learning that could prompt the development of knowledge (Brown et al., 1989; Lave, 1996). Learning through embedded activities within the social context was imperative (Brown et al., 1989; Lave, 1996). As an example, the teachers in the equityfocused PLC brought their experiences to the PLC meetings with the goal of solidifying their knowledge and their understanding of praxis. The teachers within the community co-constructed their understanding of the knowledge base and cultural beliefs (Brown et al., 1989). However, as Meg suggested, her most powerful learning experience was having other teachers observe her classroom and engage in reflections and discussions that followed in their CFG. 
Within this study, transformative learning occurred only when a teacher substantially revised or reframed his or her belief system or practice (Brookfield, 2000). In the equity-focused PLC, reflection was critical for transformative learning (Brookfield, 2000). In other words, the learner could reflect in community on the hidden agenda and power structures embedded within an educational practice yet decide to maintain one's bias or fail to act by changing one's practice. In this study, Meg acknowledged the value of communal reflection and believed happened when the members of the equity-focused PLC shared their perspectives and discussed them.

While PLCs typically highlighted shared values, reaching consensus in a diverse community was messy (Servage, 2007), especially for the equity-focused PLC in this study. Nevertheless, learning occurred when the teachers in the equity-focused PLC reached negotiated consensus through their discussions and reflections. In the case of this study, critical reflection did not appear to occur naturally, rather it needed to be prompted. The three teachers in this study noted the missed opportunities for deeper discussion and critical reflection within their equity-focused PLC. Ideally, teachers in PLCs would have engaged in exploration, reflection, and interpretation of ideas and beliefs of one another (Achinstein, 2002). Instead, members of the equity-focused PLC - like many PLCs, often ignored or avoided the discussion of difficult topics of core beliefs or equity (Westheimer, 2008).

As scholars noted, critical reflection is a requisite process for constructing new ideas and incorporating multiple perspectives within specific context such as teachers connecting their efforts to their past and future practice (Martin-Kniep, 2008; Servage, 
2008). In this study, teachers in the equity-focused PLC engaged in reflection sporadically and somewhat ineffectively (Maloney \& Konza, 2011; Westheimer, 2008). Perhaps, the externally-imposed time constraints placed on the teachers in this study limited their time for reflection and transformational learning (Servage, 2008). In addition, building and sustaining a culture of collaborative learning within PLCs has been difficult to accomplish (Servage, 2008). To bridge diverse ideas would require thoughtful attention to negotiating difficult conversations - and the skill of experienced facilitators (Achinstein, 2002, Rusch, 2005). As Meg, John, and Carmen asserted their preference for the CFG model because it relied on trained teacher-facilitators who used protocols and purposeful reflection opportunities to guide transformative learning.

\section{Conclusions}

In this section, I present my assertions based on my study's major findings. Conclusions derived were (a) teachers want a better, more productive PLC, (b) teachers want the return of the CFG, and (c) teachers find poorly developed PLCs without structure and reflection frustrating.

\section{Teachers Want to Improve}

Teachers want to be better teachers for their students. Teachers want to reach all students and are open to examining their practice and belief systems to bring about positive change in student outcomes. They believe that learning communities are an excellent platform for promoting transformational change, but they are frustrated with the model that is currently circulating in their school. 
A productive PLC must include (a) equality (in interpersonal power), (b) choice (what and how they learn), (c) teacher voice, (d) reflection, (e) dialogue, (f) praxis (reallife practice), and (g) reciprocity (expected participation) (Overstreet, 2017). Very few of these attributes appeared in the PLCs during this study. Transformative learning can take place with willing teachers, in a structured environment that is egalitarian, relevant, reflective, and trusting.

\section{Preference for CFG Model of PLCs}

Teachers believe that a viable model for an effective PLC exists in the structure of CFG. They have experienced CFGs in the past, and they long for the comeback. In their experience, the CFG has an excellent record of initiating transformational change through deep, communal reflection of problems of practice. The CFG protocols allow for equitable expression of ideas and voice, the allocated time for reflection, and the structure for community, productivity, and accountability. A teachers' willingness to explore, negotiate, and revise their beliefs is an essential requirement of transformative learning (Servage, 2008).

\section{Poorly Executed PLCs are Detrimental}

Teachers perceive a poorly executed PLC to be a waste of time and resources, frustrating, and for people of color, detrimental. Without trained facilitators to establish norms, without the attention to building deep trust, without planning a formal agenda, structure, and flow, without guided reflection, a PLC is ineffective and therefore not costeffective. But worse, an ineffectual PLC can be harmful. Teachers must recognize the emotional burden that people of color experience in groups of White people, unguided by 
knowledgeable facilitators to ensure the measured, thoughtful, and equitable treatment of issues and people. It is the responsibility of the administration and the funding districts to guarantee the structures necessary to give teachers the time and space for improving themselves and their students. Further, it is the moral obligation of the district to provide a culturally sustaining model—one that values and supports its teachers of color.

\section{Implications for Action}

My goal for the study was to examine a problem of practice regarding teachers' opportunities to reflect critically within PLCs to improve teacher practice. Because the district (and school) invested precious resources to the implementation of PLCs that allow teachers to share and refine effective teaching practices, it is important that the PLCs are effective. My research findings offer valuable insight into teachers' thinking regarding PLCs' effectiveness in changing practice and learning. In this section, I present the implications for action, stemming from my major findings.

Importantly, districts and schools should capitalize on teachers' desire to improve. Vermunt (2014) said that educational innovation succeeds or fails with the teachers that shape it. Schools and districts must provide meaningful ways for teachers to collaborate and reflect with each other. Teachers find it much more beneficial and productive than other forms of professional development. They want to evaluate and refine their craft and, with skillful facilitators, coaxed to extend examination to problematic assumptions and bias. The district and, by proxy, the schools should provide the time and space for teachers to collaborate, discuss, and reflect on their praxis. By financially supporting 
meaningful collaboration, school leaders can multiply the opportunities for teacher transformative learning and change, and therefore positive student outcomes.

School districts should immediately consider refunding the CFG model for PLCs. This should include providing the opportunity for administrators and teacher-leaders to become trained in leading CFGs, as well as funding the time and space for teachers to collaborate within the CFG. It is shortsighted to supplant CFGs - a thoughtful, effective, albeit costly, model of professional learning — with a truncated, less effective, poorlyreceived PLC. If it rarely works, it is hardly a good deal. A fully funded CFG program is an important step in attaining the purported goal of PLCs, realizing transformational change.

Student populations that suffer the injustice of inequities would benefit from focused, productive attention on institutional and systemic racism, as well as teachers' implicit biases. It is counterproductive to call for equity for all and for culturally responsive teaching practices, while policy makers short-change the critically reflective conversations that must occur within our schools. By properly funding the time and the training required for these conversations to regularly occur, schools and teachers could begin to make real headway in addressing equity disparities.

\section{Recommendations for Further Research}

In this section, I present the following recommendations for further research, based on my findings and conclusions. I focused my ideas on CFG implementation, in addition to expanded population studies on the effects of reflection within various PLCs on teacher learning, and specifically to include more teachers of color. 
The teachers I interviewed were specific and in agreement on one specific point, their preference for the CFG, Critical Friends Group, model of professional learning community. The CFG functions within a 90-minute to 2-hour format organized around structured, scripted protocols. While on the surface this level of rigidity seems antithesis to sparking innovative and mind-expanding conversation, the opposite is true. Formatting the flow of the meeting allows every member a voice and, perhaps more importantly, moments of thought and reflection. The protocols permit every person to express their thoughts and provides the sentence frames and suggestions that offer suggestions and critiques in the most palatable and nonjudgmental approaches. The CFG guidelines afford a template that holds all members accountable and focused. All members could "coach" a meeting, as well as simply present a specific problem of practice. Naturally, this amount of time and energy requires commitment and buy-in from faculties in addition to support in the form of financial recompense from school board policy makers and administrations. For these reasons specifically, I propose a study wherein rather than a CFG of 8 to 12 people, an investigator could study selfselected pairs of "critical friends" who observe, critique, reflect, and share their practices with each other. Because of the protocols, the participants must agree to participate in the CFG training, which occurs over a couple of days to familiarize themselves meeting practices. Wennergren (2016) conducted a grander-scale study based on critical-friend pairs with promising results. "A characteristic of a critical friend is the unexpected combination of...friendship built on trust, support, and affirmation and...criticism based on analysis, assessment, evaluation, and quality," (Wennergren, 2016, p.263). I believe 
the critical-friend pair arrangement is not only viable, but could stimulate real, transformational learning for teachers. Positive findings on praxis impact and teacher self-efficacy beliefs could portend the implementation of this less expensive CFG model.

Another recommendation is conducting a study with a structured reflection intervention followed by examination of efficacy beliefs and practices. My research, which studied the effects on teachers of reflection that naturally unfolded in the PLC, resulted in few reflective opportunities to explore. By expanding the study to include reflection intervention strategies, like the prompts outlined with Brookfield's (2000) CIP or an adapted version of the guided reflection protocols described by Moss, Springer, and Dehr (2008), investigators could stimulate more reflection opportunities and the effects therein.

Although case study offers valuable voices and interpretations of complicated phenomena in specific context, a mixed-methods methodology could deliver comparative data. By studying a "placebo" generic PLC group and a CFG and subsequently comparing the effects on teacher praxis and self-efficacy beliefs between the two sets.

My study included three teachers from one PLC in one school. I suggest replication and an expanded design with more teachers, more teachers of color, more PLCs, and more than one school to explore and investigate teachers' reflection in PLCs and their perceptions of the influence of PLCs in teacher learning.

Another suggestion for further research is a longitudinal examination of teachers' practices and teacher learning from direct classroom observations. External observations could mediate the effects of teacher distortion in self-reporting. 


\section{Concluding Remarks}

This study explored how critical reflection unfolded in an equity PLC and how teachers perceived it to influence their teacher practices and beliefs. My study showed that any reflection at all occurred sporadically and ineffectively. Although the teachers professed the importance of reflection and considered it almost "a moral directive" to engage in it as an educator, they universally spoke to the dearth of opportunity to reflect meaningfully. While the teachers recognized the PLC platform as a viable venue for initiating teacher learning, they were very clear that the CFG model was superior in every way.

Teachers in my study were frustrated. In addition to the multitude of responsibilities, interactions, and duties on a teacher's daily plate, several requiredrarely effective-- PLCs every month was too much. Teachers wanted focused, structured, equitable, guided, and productive meetings. They felt the CFGs met those requirements and more. Their prior experience with CFG meetings were enjoyable and beneficial.

The study revealed that while teachers were willing to put in the hard work of transformational change, they were resentful of mandated "false learning communities," as Carmen called them. They reported their annoyance by $45-$ to 90 -minute meetings to address operational technicalities better suited to emails. The teachers wanted substantive meetings that made it worth their time.

I concluded that teachers wanted to improve and further, believed that PLCs have the potential to improve their craft. However, they unanimously felt the current model of 
PLC fell short because of three factors (a) the lack of trained facilitator, (b) the lack of structure, and (c) the lack of opportunities for reflection. The teachers communicated these insufficiencies during their interviews, and I corroborated it through researcher observations. To increase the impact on teacher learning, I recommend that the district put in place policies to fund the more productive version of PLC - the CFG. I discuss implications of these findings in recommendations for administrators and policy makers, as well as future research. 


\section{References}

Achinstein, B. (2002). Conflict amid community: The micropolitics of teacher collaboration. Teachers College Record, 104, 421-455.

Anderson, J. R., Reder, L. M., \& Simon, H. A. (1996). Situated learning and education. Educational Researcher, 25(3), 5-11. doi.org/10.3102/0013189X025004005

Attard, K. (2012). Public reflection within learning communities: An incessant type of professional development. European Journal of Teacher Education, 33, 249270.

Awkard, T. (2017). The power of reflective action to build teacher efficacy. Phi Delta Kappan, 98(6), 53-57. doi.org/10.1177/0031721717696479

Ayers, W. C. (2001). To teach: The journey of a teacher (2nd ed.). New York, NY: Teachers College Press.

Badia, G. (2017). Combing critical reflection and action research to improve pedagogy. Libraries and the Academy, 17, 695-720.

Bakkenes, I., Vermunt, J. D., \& Wubbles, T. (2010). Teacher learning in the context of educational innovation: Learning activities and learning outcomes of experienced teachers. Learning and Instruction, 20, 533-548.

Banks, J., Cochran-Smith, M., Moll, L., Richert, A., Zeichner, K., LePage, P.... Duffy, H. (2005). Teaching diverse learners. In L. Darling-Hammond \& J. Bransford (Eds.), Preparing teachers for a changing world: What teachers should learn and be able to do. (pp. 232-274). San Francisco, CA: Jossey-Bass. 
Bereiter, C. (1994). Constructivism, socioculturalism, and Popper's world 3. Educational Researcher, 23(7), 21-23.

Berliner, D. C., \& Biddle, B. J. (1995). The manufactured crisis: Myths, fraud, and the attack on America's public schools. Reading, MA: Addison-Wesley.

Bolman, L. G., \& Deal, T. E. (2008). Reframing organizations: Artistry, choice, and leadership (4th ed.). San Francisco, CA: Jossey-Bass.

Bray-Clark, N. \& Bates, R. (2003). Self-efficacy beliefs and teacher effectiveness: Implications for professional development. The Professional Educator, 26(1), 1322.

Brazas, C. \& McGeehan, C. (2020). What White colleagues need to understand: White supremacy doesn't stop at the teachers' lounge door. Teaching Tolerance, 64, 5658.

Brookfield, S. (1995). Becoming a critically reflective teacher. San Francisco, CA: Jossey-Bass.

Brookfield, S. (2000). Transformative learning as ideology critique. In J. Mezirow (Ed.), Learning as transformation: Critical perspectives on a theory in progress (pp. 125-148). San Francisco, CA: Jossey-Bass.

Brown, J. S., Collins, A., \& Duguid, P. (1989). Situated cognition and the culture of learning. Educational Researcher, 18(1), 32-42. doi:10.3102/0013189X018001032

Calderwood, P. (2000). Learning community: Finding common ground in difference. New York, NY: Teachers College Press. 
Campbell, E. (2008). The ethics of teaching as a moral profession. Curriculum Inquiry, $38,357-385$.

Caskey, M. M., \& Carpenter, J. (2012). Organization models for teacher learning. Middle School Journal, 43(5), 52-62.

Cobb, P. (1994). Where is the mind? Constructivist and sociocultural perspectives on mathematical development. Educational Researcher, 23(7), 13-20.

Cochran-Smith, M. \& Lytle, S. L. (2009). Inquiry as stance: Practitioner research for the next generation. New York, NY: Teachers College Press.

Creswell, J. W. (2007). Qualitative inquiry \& research design: Choosing among five approaches (2nd ed.). Thousand Oaks, CA: SAGE.

Darling-Hammond, L., \& Bransford, J. (Eds.). (2005). Preparing teachers for a changing world: What teachers should learn and be able to do. San Francisco, CA: Jossey-Bass.

Darling-Hammond, L., \& McLaughlin, M. W. (2011). Policies that support professional development in an era of reform. Phi Delta Kappan, 92(6), 81-92.

Darling-Hammond, L., \& Sykes, G. (Eds.). (1999). Teaching as the learning profession: Handbook of policy and practice. San Francisco, CA: Jossey-Bass.

Deissler, C. H. (2008). Changing beliefs versus changing concepts: Transformational learning as a tool for investigating and encouraging teacher belief change. In Educational Media and Technology Yearbook, 33, 91-99. Westport, CT: ABCClio-Greenwood. 
Denzin, N. K., \& Lincoln, Y. S. (2005). The SAGE handbook of qualitative research (3rd ed.). Thousand Oaks, CA: SAGE.

Dewey, J. (1910). How we think. Boston, MA: D.C. Heath \& Company.

Dewey, J. (1916). Democracy \& education (2009 Digitalized ed.). Gearhart, OR: Watchmaker Publishing for Merchant Books.

Dewey, J. (1933). How we think: A restatement of the relation of reflective thinking to the educative process (Revised ed.). Boston, MA: D.C. Heath \& Company.

DuFour, R. (2004). What is a "professional learning community"? Educational Leadership, 61(8), 6-11.

DuFour, R., \& Eaker, R. (1998). Professional learning communities at work: Best practices for enhancing student achievement. Bloomington, IN: Solution Tree.

Durden, T. R. \& Truscott, D. M. (2013). Critical reflectivity and the development of new culturally relevant teachers. Multicultural Perspectives, 15(2), 73-80.

Ernest, P. (1993). Constructivism, the psychology of learning, and the nature of mathematics: Some critical issues. Science and Education, 2, 87-93.

Ernest, P. (1994). Varieties of constructivism: Their metaphors, epistemologies, and pedagogical implications. Hiroshima Journal of Mathematics Education, 2, 1-14.

Feldman, J., \& Fataar, A. (2014). Conceptualizing the setting up of a professional learning community for teachers' pedagogical learning, South African Journal of Higher Education, 28, 1525-1540. 
Fenwick, T. J. (2000). Expanding conceptions of experiential learning: A review of the five contemporary perspectives on cognition. Adult Education Quarterly, 50, 243-272. doi:10.1177/07417130022087035

Ferguson, R. F. (1991). Racial patterns in how school and teacher quality affect achievement and earnings. Challenge, 2(1), 1-35.

Fishman, S. M., \& McCarthy, L. (1998). John Dewey and the challenge of classroom practice. New York, NY: Teachers College Press.

Fullan, M. (1995). The school as learning organization: Distant dreams. Theory Into Practice, 34, 230-235.

Fullan, M. (2006). Leading professional learning. School Administrator, 63(10), 10-14.

Gay, G. (2002). Preparing for culturally responsive teaching. Journal of Teacher Education, 53, 106-116.

Graham, P. (2007). Improving teacher effectiveness through structured collaboration: A case study of a professional learning community. Research in Middle Level Education Online, 31(1), 1-17.

Guba, E. G., \& Lincoln, Y. S. (2005). Paradigmatic controversies, contradictions, and emerging confluences. In N. K. Denzin \& Y. S. Lincoln (Eds.), The SAGE handbook of qualitative research (3rd ed., pp. 191-216). Thousand Oaks, CA: SAGE.

Hammond, Z. (2015). Culturally responsive teaching and the brain: Promoting authentic engagement and rigor among culturally and linguistically diverse students. Thousand Oaks, CA: Corwin. 
Hoffman-Kipp, P., Artiles, A. J., \& López-Torres, L. (2003). Beyond reflection: Teacher learning as praxis. Theory Into Practice, 42, 248-254.

Hord, S. M. (2004). Professional Learning Communities: An overview. In S. M. Hord (Ed.), Learning together, leading together: Changing schools through professional learning communities (pp. 5-14). Columbia University, NY: Teachers College Press.

Jay, J. K., \& Johnson, K. L. (2002). Capturing complexity: A typology of reflective practice for teacher education. Teaching and Teacher Education, 18, 73-85.

Jones, C. M., \& Thessin, R. A. (2015). A review of the literature related to the change process schools undergo to sustain PLCs. Planning and Changing, 46(1/2), 193211.

Kahne, J. (1994). Democratic communities, equity, and excellence: A Deweyan reframing of educational policy analysis. Educational Evaluation and Policy Analysis, 16, 233-248.

Kim, H., \& Hannafin, M. J. (2008). Situated case-base knowledge: An emerging framework for prospective teacher learning. Teaching and Teacher Education, 24, 1837-1845.

King, K. P. (2011). Professional learning in unlikely spaces: Social media and virtual communities as professional development. International Journal of Emerging Technologies in Learning, 6(4), 40-46.

Kuhn, T. (1996). The structure of scientific revolutions (originally published 1962). Chicago, IL: University of Chicago Press. 
Ladson-Billings, G. (2006). From the achievement gap to the education debt: Understanding achievement in U.S. schools. Educational Researcher, 35(7), 312. Retrieved from http://www.jstor.org/stable/3876731

Larrivee, B. (2000). Transforming teacher practice: Becoming the critically reflective teacher. Reflective Practice, 1, 293-307.

Larrivee, B. (2008). Development of a tool to access teachers' level of reflective practice. Reflective Practice, 9, 341-360.

Lave, J. (1996). Teaching as learning in practice. Mind, Culture \& Activity, 3, 149-164.

Lave, J., \& Wenger, E. (1991). Learning in doing: Social, cognitive and computational perspectives. New York, NY: Cambridge University Press.

Laverick, L. T. (2017). Secondary teachers' understanding and use of reflection: An exploratory study. American Secondary Education, 45(2), 56-68.

Leithwood, K., \& Jantzi, D. (2008). Linking leadership to student learning: The contributions of leader efficacy. Educational Administration Quarterly, 44, 496528.

Linder, R. A., Post, G., \& Calabrese, K. (2012). Professional learning communities: Practices for successful implementation. Delta Kappa Gamma Bulletin, 78(3), $13-22$.

Liu, K. (2015). Critical reflection as a framework for transformative learning in teacher education. Educational Review, 67, 135-157. 
Liu, K. (2017). Creating a dialogic space for prospective teacher critical reflection and transformative learning. Reflective Practice, 18, 805-820. doi:10.1080/14623943.2017.1361919.

Long, K. (2012). Changing teachers' practice through critical reflection on pedagogy. The International Journal of Interdisciplinary Social Sciences, 6(4), 145-159.

Louis, K. S., \& Marks, W. H. (1998). Does professional learning community affect classroom? Teachers' work and student experiences in restructuring schools. American Journal of Education, 106, 532-575.

Love, A., \& Kruger, A. C. (2005). Teacher beliefs and student achievement in urban schools serving African American students. Journal of Educational Research, 99, 87-98.

Mahnken, K. (2017, October 31). $61 \%$ of teachers stressed out say mental health is not good in new national survey. The 74. Retrieved from https://www.the74million.org/61-of-teachers-stressed-out-58-say-mental-healthis-not-good-in-new-national-survey/

Maloney, C., \& Konza, D. (2011). A case study of teachers' professional learning: Becoming a community of professional learning or not? Issues in Educational Research, 21, 75-87.

Martin-Kniep, G. O. (2008). Communities that learn, lead, and last: Building and sustaining educational expertise. San Francisco, CA: Jossey-Bass.

Maxwell, J. A. (2005). Qualitative research design: An interactive approach ( $2 \mathrm{nd}$ ed.). Thousand Oaks, CA: SAGE. 
McComish, D., \& Parsons, J. (2013). Transformational learning and teacher collaborative communities. New Zealand Journal of Teachers' Work, 10, 239-245.

McLaren, P. (1994). Life in schools: An introduction to critical pedagogy in the foundations of education (2nd ed.). New York, NY: Longman.

Meier, D. (2000). Will standards save public education? Boston, MA: Beacon Press.

Merriam, S. B. (1998). Qualitative research and case study applications in education (2nd ed.). San Francisco, CA: Jossey-Bass.

Mezirow, J. (1990). How critical reflection triggers transformative learning. In Mezirow (Ed.), Fostering critical reflection in adulthood (pp. 1-20). San Francisco, CA: Jossey-Bass.

Mezirow, J. (2000). Learning as transformation: Critical perspectives on a theory in progress. San Francisco, CA: Jossey-Bass.

Mezirow, J. (2003). Transformational learning as discourse. Journal of Transformative Education, 1, 58-63.

Morgan, G. (2006). Images of organization (Updated ed.). Thousand Oaks, CA: SAGE.

Moss, G., Springer, T., \& Dehr, K. (2008). Guided reflection protocol as narrative inquiry and teacher professional development. Reflective Practice, 9, 497-508. doi: $10.1080 / 14623940802431739$

National Center for Education Statistics. (2016). The state of racial diversity in the educator workforce, U.S. Department of Education, Office of Planning, Evaluation and Policy Development, Policy and Program Studies Service, Washington, D.C. Retrieved from 
http://www2.ed.gov/rschstat/eval/highered/racial-diversity/state-racialdiversityworkforce.pdf

National Commission on Excellence in Education. (1983). A nation at risk: The imperative for educational reform. Washington, DC: U.S. Department of Education.

National Score Reform Faculty. (n.d.). A Critical Friends Group community. Retrieved from https://nsrfharmony.org/faq-items/cfgvsplc/

Overstreet, M. (2017). Culture at the core: Moving from professional development to professional learning. Journal of Ethnographic \& Qualitative Research, 11, 199214.

Owen, S. (2014). Teacher professional learning communities: Going beyond contrived collegiality toward challenging debate and collegial learning and professional growth. Australian Journal of Adult Learning, 54(2), 54-77.

Pancucci, S. (2008). A retrospective analysis of a professional learning community: How teachers' capacities shaped it. International Journal of Social Sciences, 3(1), 62 69.

Peters, G. (2016). How we can bridge the culture gap. Journal of Staff Development, 37(5), 12-22. Retrieved from www.leaningforward.org

Printy, S. M. (2008). Leadership for teacher learning: A community of practice perspective. Educational Administration Quarterly, 44, 187-226. 
Riveros, A., Newton, P., \& Burgess, D. (2012). A situated account of teacher agency and learning: Critical reflections on professional learning communities. Canadian Journal of Education, 35(1), 475-514.

Rodgers, C. (2002). Defining reflection: Another look at John Dewey and reflective thinking. Teachers College Record, 104, 842-866.

Ronfeldt, M., Owens Farmer, S, McQueen, K., \& Grissom, J. A. (2015). Teacher collaboration in instructional teams and student achievement. American Educational Research Journal, 52, 475-514.

Rusch, E. A. (2005). Institutional barriers to organizational learning in school systems: The power of silence. Educational Administration Quarterly, 41, 83-120. doi:10.1177/0013161X04269546

Saldaña, J. (2009). The coding manual for qualitative researchers. Thousand Oaks, CA: SAGE.

Šarić, M., \& Šteh, B. (2017). Critical reflection in the professional development of teachers: Challenges and possibilities. Center for Educational Policy Journal, $7(3), 67-85$.

Senge, P., \& Lannon-Kim, C. (1991). Recapturing the spirit of learning through a systems approach. School Administrator, 48(9), 8-13.

Servage, L. (2007). Making space for critical reflection in professional learning communities. Education Canada, 47(1), 14-17. 
Servage, L. (2008). Critical and transformative practices in professional learning communities. Teacher Education Quarterly, 35(1), 63-77.

Servage, L. (2009). Who is the "professional" in a professional learning community? An exploration of teacher professionalism in collaborative professional development settings. Canadian Journal of Education, 32(1), 149-171.

Sfard, A. (1998). On two metaphors for learning and the dangers of choosing just one. Educational Researcher, 27(2), 4-13.

Sims, R. L., \& Penny, G. R. (2014). Examination of a failed professional learning community. Journal of Education and Training Studies, 3(1), 39-45.

Siwatu, K. O. (2007). Preservice teachers' culturally responsive teaching self-efficacy and outcome beliefs. Teacher and Teacher Education, 23, 1086-1101.

Siwatu, K. O. (2011). Preservice teachers' culturally responsive teaching self-efficacyforming experiences: A mixed methods study. Journal of Educational Research, 104(5), 306-369.

Smeed, J., Kimber, M., Millwater, J., \& Ehrich, L. (2009). Power over, with and through: Another look at micropolitics. Leading \& Managing, 15(1), 26-41.

Steeg, S. (2016). A case study of teacher reflection: Examining teacher participation in a video-based professional learning community. Journal of Language and Literacy Education, 12(1), 122-140.

Stein, D. S., \& Imel, S. (2002). Adult learning in community: Themes and threads. New Directions for Adult and Continuing Education, 95(Fall), 93-97. 
Thiers, N. (2017). Educators deserve better: A conversation with Richard DuFour. Educational Leadership, 73(8), 10-16. Retrieved from http://www.ascd.org/publications/educationalleadership/may16/vol73/num08/Educators-Deserve-Better@-A-Conversationwith-Richard-DuFour.aspx

Thompson, C. L., \& Zeuli, J. S. (1999). The frame and the tapestry: Standards-based reform and professional development. In L. Darling-Hammond \& G. Sykes (Eds.), Teaching as the learning profession: Handbook of policy and practice (pp. 341-375). San Francisco, CA: Jossey-Bass.

Tillema, H., \& Orland-Barak, L., (2006). Constructing knowledge in professional conversations: The role of beliefs on knowledge and knowing. Learning and Instruction, 16, 592-608.

Tschannen-Moran, M., \& Woolfolk Hoy, A., (2001). Teacher efficacy: Capturing an elusive construct. Teacher and Teacher Education, 17, 783-805.

Tyack, D., \& Cuban, L. (1995). Tinkering toward utopia: A century of public school reform. Cambridge, MA: Harvard University Press.

Valli, L. (1994, April). Professional developmental schools: An opportunity to reconceptualize schools and teacher education as empowering learning communities. Paper presented at the International Seminar on Teacher Education (ISTE) Annual Meeting, Maastricht, Netherlands. 
Vangrieken, K., Meredith, C., Packer, T., \& Kyndt, E. (2017). Teacher communities as a context for professional development: A systematic review. Teaching and Teacher Education, 61, 47-59.

Van Lare, M. D., \& Brazer, S. D. (2013). Analyzing learning in professional learning communities: A conceptual framework. Leadership and Policy in Schools, 12, 374-396.

Vermunt, J. (2014). Teacher learning and professional development. In S. KrolakSchwerdt, S. Glock, \& M. Böhmer (Eds.), Teachers' professional development: Assessment, training, and learning, (pp.79-95). Rotterdam, Netherlands: Sense.

Vescio, V., Ross, D., \& Adams, A. (2008). A review of research on the impact of professional learning communities on teaching practice and student learning. Teaching and Teacher Education 24, 80-91.

von Glasersfeld, E. (1991). Radical constructivism in mathematics education. Retrieved from http://search.ebscohost.com/login.aspx?direct=true \&db=eric\&AN=ED341564\&si te=ehost-live

Wallerstein, N., \& Duran, B. (2003). The conceptual, historical, and practice roots of community based participatory research and related participatory traditions. In M. Minkler \& N. Wallerstein (Eds.), Community based participatory research for health (pp. 27-52). San Francisco, CA: Jossey-Bass.

Wennergren, A. (2016). Teachers as learners-with a little help from a critical friend. Educational Action Research, 24(2), 260-279. 
Westheimer, J. (2008). Learning among colleagues: Teacher community and the shared enterprise of education. In M. Cochran-Smith, D. Feiman-Nemser, \& J. McIntyre (Eds.), Handbook of teacher education: Enduring questions in changing contexts (3rd ed., pp. 756-783). New York, NY: Routledge.

Will, M. (2017, October 31). Educators are more stressed at work than average people, survey finds [Web log post]. Retrieved from http://blogs.edweek.org/teachers/teaching_now/2017/10/educator_stress_aft_bat.h tml?print $=1$

Wolcott, H. F. (2008). Ethnography: A way of seeing (2nd ed.). Lanham, MD: Altamira Press.

Wood, D. (2007a). Teachers' learning communities: Catalyst for change or a new infrastructure for the status quo? Teachers College Record, 109, 699-739.

Wood, D. R. (2007b). Professional learning communities: Teachers, knowledge, and knowing. Theory Into Practice, 46, 281-290. doi:10.1080/00405840701593865

Yin, R. K. (2009). Case study research: Design and methods (4th ed.). Thousand Oaks, CA: SAGE.

Zeichner, K. M. (1993). Connecting genuine teacher development to the struggle for social justice. Journal of Education for Teaching, 19(1), 5-20.

Zeichner, K. M., \& Liston, D. P. (1996). Reflective teaching: An introduction. Mahwah, NJ: Erlbaum. 


\section{Appendix A}

\section{Informed Consent Form}

Teachers' Critical Reflection in an Equity-Focused Professional Learning Community: A Case Study

You are invited to participate in a research study conducted by Patrizia Lina Mastne from the Department of Curriculum and Instruction at Portland State University. The researcher hopes to gain an understanding (a) if and how high school teachers engage in critical reflection in their PLC, and (b) if and how critical reflection impacts teachers' efficacy beliefs, teacher learning, and teacher praxis. The study will be conducted in partial fulfillment of the requirements for a doctor in education (Ed. D) degree, under supervision of Dr. Micki M. Caskey. If you decide to participate, you will be asked on respond to two brief surveys requiring approximately 15 minutes each to complete. The first survey would assess your current level of engagement with professional reflection. The second survey would give a baseline of your teacher efficacy beliefs. In addition, you will be invited to participate in two 30 to 45 -minute interviews and two 60-minute focus group discussion, all of which will be audio recorded and transcribed. You will be given access to the transcriptions for your edits and approval.

Your participation is important and valuable. While participating in this study, it is possible that you may gain additional insights about teaching and your own teacher efficacy beliefs and teacher learning. You may not receive any direct benefit from taking part in this study, but the study may increase knowledge which may help improve praxis in the future. The findings also might be useful to support your own professional development. The risk to teachers' reputation, job, or overall well-being by participating in this research is equivalent to the risk teachers would encounter in engaging in any school-based professional development activity. Feelings of discomfort from reflection may occur.

Any information that is obtained in connection with this study which could be linked to you or your identity will be kept confidential. To maintain confidentiality, the researcher will keep the records in a locked file cabinet and on a password protected file on a laptop computer in a secure office.

Your participation is voluntary. You do not have to take part in this study, and it will not affect your relationship with your colleagues or supervisors. You may also withdraw from this study at any time without reprisal in any form. Without giving any reason, you may also choose not to respond to a particular question during the research project. Please be assured that there is never a right or wrong answer.

If you have questions about the study itself, contact Patrizia Mastne, 615 SW Harrison Street, Portland, OR 97201, 503-725-4722, pmastne@pdx.edu or the Human Subjects Research Review Committee, Research \& Strategic Partnerships, PO BOX 751, Portland, OR 97207, 503- 725-4288, hsrrc@list.pdx.edu, or Dr. Micki M. Caskey, caskeym@pdx.edu 
Your signature indicates that you have read and understand the above information and agree to take part in this study. Please understand that you may withdraw your consent at any time without penalty, and that, by signing, you are not waiving any legal claims, rights, or remedies. The researcher will provide you with a copy of this form for your own record. 


\section{Appendix B}

\section{Teacher Questionnaire}

\begin{tabular}{|c|c|c|c|}
\hline $\begin{array}{r}\text { Survey of Reflective Practice: A Tool for } \\
\text { Practitioner Se }\end{array}$ & $\begin{array}{l}\text { Assessing De } \\
\text {-Assessment }\end{array}$ & elopment & a Reflective \\
\hline Practice Indicators & \multirow{3}{*}{\multicolumn{3}{|c|}{ Assessment Criteria }} \\
\hline For each indicator, select the rating you & & & \\
\hline I am a teacher/teacher candidate who: & & & \\
\hline LEVEL 1: PRE-REFLECTION & Frequently & Sometimes & Infrequently \\
\hline \multicolumn{4}{|l|}{$\begin{array}{l}\text { Operates in survival mode, reacting } \\
\text { automatically without consideration of } \\
\text { alternative responses }\end{array}$} \\
\hline \multicolumn{4}{|l|}{$\begin{array}{l}\text { Enforces preset standards of operation } \\
\text { without adapting or restructuring based on } \\
\text { students' responses }\end{array}$} \\
\hline \multicolumn{4}{|l|}{$\begin{array}{l}\text { Does not support beliefs and assertions with } \\
\text { evidence from experience, theory or } \\
\text { research }\end{array}$} \\
\hline \multicolumn{4}{|l|}{$\begin{array}{l}\text { Is willing to take things for granted without } \\
\text { questioning }\end{array}$} \\
\hline \multicolumn{4}{|l|}{$\begin{array}{l}\text { Is preoccupied with management, control } \\
\text { and student compliance }\end{array}$} \\
\hline \multicolumn{4}{|l|}{$\begin{array}{l}\text { Fails to recognize the interdependence } \\
\text { between teacher and student actions }\end{array}$} \\
\hline \multicolumn{4}{|l|}{$\begin{array}{l}\text { Views student and classroom circumstances } \\
\text { as beyond the teacher's control }\end{array}$} \\
\hline \multicolumn{4}{|l|}{$\begin{array}{l}\text { Attributes ownership of problems to students } \\
\text { or others }\end{array}$} \\
\hline \multicolumn{4}{|l|}{ Fails to consider differing needs of learners } \\
\hline \multicolumn{4}{|l|}{ Sees oneself as a victim of circumstances } \\
\hline \multicolumn{4}{|l|}{$\begin{array}{l}\text { Dismisses students' perspectives without } \\
\text { consideration }\end{array}$} \\
\hline \multicolumn{4}{|l|}{$\begin{array}{l}\text { Does not thoughtfully connect teaching } \\
\text { actions with student learning or behavior }\end{array}$} \\
\hline $\begin{array}{l}\text { Describes problems simplistically or } \\
\text { unidimensionally }\end{array}$ & & & \\
\hline
\end{tabular}




\begin{tabular}{|l|l|l|l|}
\hline $\begin{array}{l}\text { Does not see beyond immediate demands of } \\
\text { a teaching episode }\end{array}$ & & & \\
\hline \multicolumn{1}{|c|}{ LEVEL 2: SURFACE REFLECTION } & Frequently & Sometimes & Infrequently \\
\hline $\begin{array}{l}\text { Limits analysis of teaching practices to } \\
\text { technical questions about teaching } \\
\text { techniques }\end{array}$ & & & \\
\hline $\begin{array}{l}\text { Modifies teaching strategies without } \\
\text { challenging underlying assumptions about } \\
\text { teaching and learning }\end{array}$ & & \\
\hline $\begin{array}{l}\text { Fails to connect specific methods to } \\
\text { underlying theory }\end{array}$ & & \\
\hline $\begin{array}{l}\text { Supports beliefs only with evidence from } \\
\text { experience }\end{array}$ & & & \\
\hline $\begin{array}{l}\text { Provides limited accommodations for } \\
\text { students' different learning styles }\end{array}$ & & \\
\hline $\begin{array}{l}\text { Reacts to student responses differentially but } \\
\text { fails to recognize patterns }\end{array}$ & & & \\
\hline $\begin{array}{l}\text { Adjusts teaching practices only to current } \\
\text { situation without developing a long-term } \\
\text { plan }\end{array}$ & & & \\
\hline $\begin{array}{l}\text { Implements solutions to problems that focus } \\
\text { only on short-term results }\end{array}$ & & & \\
\hline $\begin{array}{l}\text { Adjusts based on past experience } \\
\text { practices but not general policies or } \\
\text { practices }\end{array}$ & & \\
\hline $\begin{array}{l}\text { Provides some differentiated instruction to } \\
\text { address students' individual differences }\end{array}$ & & \\
\hline & & \\
\hline
\end{tabular}




\section{Survey of Reflective Practice: A Tool for Assessing Development as a Reflective Practitioner}

\begin{tabular}{|c|c|c|c|}
\hline $\begin{array}{c}\text { Practice Indicators } \\
\text { For each indicator, select the rating you } \\
\text { think best represents your current practice. } \\
\text { I am a teacher/teacher candidate who: }\end{array}$ & \multicolumn{3}{|c|}{ Assessment Criteria } \\
\hline $\begin{array}{l}\text { LEVEL 3: PEDAGOGICAL } \\
\text { REFLECTION }\end{array}$ & Frequently & Sometimes & Infrequently \\
\hline \multicolumn{4}{|l|}{$\begin{array}{l}\text { Analyzes relationship between teaching } \\
\text { practices and student learning }\end{array}$} \\
\hline \multicolumn{4}{|l|}{ Strives to enhance learning for all students } \\
\hline \multicolumn{4}{|l|}{$\begin{array}{l}\text { Seeks ways to connect new concepts to } \\
\text { students' prior knowledge }\end{array}$} \\
\hline \multicolumn{4}{|l|}{$\begin{array}{l}\text { Has genuine curiosity about the } \\
\text { effectiveness of teaching practices, leading } \\
\text { to experimentation and risk-taking }\end{array}$} \\
\hline \multicolumn{4}{|l|}{$\begin{array}{l}\text { Engages in constructive criticism of one's } \\
\text { own teaching }\end{array}$} \\
\hline \multicolumn{4}{|l|}{$\begin{array}{l}\text { Adjusts methods and strategies based on } \\
\text { students' relative performance }\end{array}$} \\
\hline \multicolumn{4}{|l|}{$\begin{array}{l}\text { Analyzes the impact of task structures, such } \\
\text { as cooperative learning groups, partner, peer } \\
\text { or other groupings, on students' learning }\end{array}$} \\
\hline \multicolumn{4}{|l|}{$\begin{array}{l}\text { Searches for patterns, relationships and } \\
\text { connections to deepen understanding }\end{array}$} \\
\hline \multicolumn{4}{|l|}{$\begin{array}{l}\text { Has commitment to continuous learning and } \\
\text { improved practice }\end{array}$} \\
\hline \multicolumn{4}{|l|}{$\begin{array}{l}\text { Identifies alternative ways of representing } \\
\text { ideas and concepts to students }\end{array}$} \\
\hline \multicolumn{4}{|l|}{$\begin{array}{l}\text { Recognizes the complexity of classroom } \\
\text { dynamics }\end{array}$} \\
\hline \multicolumn{4}{|l|}{$\begin{array}{l}\text { Acknowledges what student brings to the } \\
\text { learning process }\end{array}$} \\
\hline \multicolumn{4}{|l|}{$\begin{array}{l}\text { Considers students' perspectives in decision } \\
\text { making }\end{array}$} \\
\hline $\begin{array}{l}\text { Sees teaching practices as remaining open } \\
\text { to further investigation }\end{array}$ & & & \\
\hline
\end{tabular}




\begin{tabular}{|l|l|l|l|}
\hline LEVEL 4: CRITICAL REFLECTION & Frequently & Sometimes & Infrequently \\
\hline $\begin{array}{l}\text { Views practice within the broader } \\
\text { sociological, cultural, historical, and } \\
\text { political contexts }\end{array}$ & & & \\
\hline $\begin{array}{l}\text { Considers the ethical ramifications of } \\
\text { classroom policies and practices }\end{array}$ & & & \\
\hline $\begin{array}{l}\text { Addresses issues of equity and social justice } \\
\text { that arise in and outside of the classroom }\end{array}$ & & & \\
\hline $\begin{array}{l}\text { Challenges status quo norms and practices, } \\
\text { especially with respect to power and control }\end{array}$ & & & \\
\hline Observes self in the process of thinking & & & \\
\hline $\begin{array}{l}\text { Is aware of incongruence between beliefs } \\
\text { and actions and acts to rectify }\end{array}$ & & & \\
\hline $\begin{array}{l}\text { Acknowledges the social and political } \\
\text { consequences of one's teaching }\end{array}$ & & & \\
\hline $\begin{array}{l}\text { Is an active inquirer, both critiquing current } \\
\text { conclusions and generating new hypotheses }\end{array}$ & & & \\
\hline $\begin{array}{l}\text { Challenges assumptions about students and } \\
\text { expectations for students }\end{array}$ & & & \\
\hline Suspends judgments to consider all options & & & \\
\hline $\begin{array}{l}\text { Recognizes assumptions and premises } \\
\text { underlying beliefs }\end{array}$ & & & \\
\hline Calls commonly-held beliefs into question & & & \\
\hline $\begin{array}{l}\text { Acknowledges that teaching practices and } \\
\text { policies can either contribute to, or hinder, } \\
\text { the realization of a more just and humane } \\
\text { society }\end{array}$ & & & \\
\hline $\begin{array}{l}\text { Encourages socially responsible actions in } \\
\text { their students }\end{array}$ & & & \\
\hline Ad & & & \\
\hline
\end{tabular}

Adapted from Larrivee, B. (2008). Development of a tool to access teachers' level of reflective practice. Reflective Practice, 9(3), 341-360. 


\section{Appendix C}

\section{Culturally Responsive Teaching Self-Efficacy Scale}

Rate how confident you are in your ability to successfully accomplish each of the tasks listed below. Each task is related to teaching. Please rate your degree of confidence by recording a number from 0 (no confidence at all) to 100 (completely confident). Remember that you may use any number between 0 and 100.

\begin{tabular}{ccccccccc}
\hline $0 \quad 10$ & 20 & 30 & 40 & $\begin{array}{c}50 \\
\text { Moderately } \\
\text { Confident }\end{array}$ & & & &
\end{tabular}

\section{I am able to:}

1. adapt instruction to meet the needs of my students.

2. obtain information about my students' academic strengths.

3. determine whether my students like to work alone or in a group.

4. determine whether my students feel comfortable competing with other students.

5. identify ways that the school culture (e.g., values, norms, and practices) is different from my students' home culture.

6. implement strategies to minimize the effects of the mismatch between my students' home culture and the school culture.

7. assess student learning using various types of assessments.

8. obtain information about my students' home life.

9. build a sense of trust in my students.

10. establish positive home-school relations.

11. use a variety of teaching methods.

12. develop a community of learners when my class consists of students from diverse backgrounds.

13. use my students' cultural background to help make learning meaningful.

14. use my students' prior knowledge to help them make sense of new information.

15. identify ways how students communicate at home may differ from the school norms.

16. obtain information about my students' cultural background.

17. teach students about their cultures' contributions to science.

18. greet English Language Learners with a phrase in their native language. 
19. design a classroom environment using displays that reflects a variety of cultures.

20. develop a personal relationship with my students.

21. obtain information about my students' academic weaknesses.

22. praise English Language Learners for their accomplishments using a phrase in their native language.

23. communicate with parents regarding their child's educational progress.

24. identify ways that standardized tests may be biased towards linguistically diverse students.

25. structure parent-teacher conferences so that the meeting is not intimidating for parents.

26. help students to develop positive relationships with their classmates.

27. revise instructional material to include a better representation of cultural groups.

28. critically examine the curriculum to determine whether it reinforces negative cultural stereotypes.

29. design a lesson that shows how other cultural groups have made use of mathematics.

30. model classroom tasks to enhance English Language Learner's understanding.

31. communicate with the parents of English Language Learners regarding their child's achievement.

32. help students feel like important members of the classroom.

33. identify ways that standardized tests may be biased towards culturally diverse students.

34. use a learning preference inventory to gather data about how my students like to learn.

35. use examples that are familiar to students from diverse cultural backgrounds.

36. explain new concepts using examples that are taken from my students' everyday lives.

37. obtain information regarding my students' academic interests.

38. use the interests of my students to make learning meaningful for them.

39. implement cooperative learning activities for those students who like to work in groups.

40. design instruction that matches my students' developmental needs.

41. teach students about their cultures' contributions to society. 


\section{Appendix D}

\section{Larrivee Statement of Permission to Use}

Survey of Reflective Practice: A Tool for Assessing Development as a Reflective Practitioner

The Survey of Reflective Practice consists of a three-part tool including the:

(1) Facilitator Assessment

(2) Self Assessment

(3) Action Plan for Improved Practice

I, Barbara Larrivee, hereby grant permission under the conditions specified below to use the Survey of Reflective Practice: A Tool for Assessing Development as a Reflective Practitioner, to:

Name: Patrizia Mastne

Institution: Portland State University

Address: 19936 SW Luree St., Beaverton, OR 97003

Phone no.: 503-550-7550

Email: pmastne@pdx.edu

As a condition for using the Survey, the above named agrees to the following conditions:

1. This permission is granted for research purposes only.

2. If changes are made to the Survey, the citation must say "adapted from."

3. A copy of the final format in which you intend to make use of the Survey must be e-mailed to me prior to its use and/or dissemination.

4. Within 60 days of completion of the research, provide the raw data collected for the potential purpose of pooling data to conduct further research on the Survey. 5. Within 60 days of completion of the research, provide a written summary of findings including a by-item analysis.

I agree to these conditions to use the Survey.

Patrizia Lina Mastne November 1, 2018

Survey User

Date 


\section{Appendix E}

\section{Permission to Use Instrument: Culturally Responsive Teaching Self- Efficacy Scale}

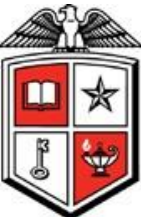

TEXAS TECH UNIVERSITY College of Education

Dear Researcher:

You have my permission to use the Culturally Responsive Teaching SelfEfficacy Scale, the Culturally Responsive Teaching Outcome Expectations Scale, and/or the Culturally Responsive Classroom Management SelfEfficacy Scale in your research. A copy of the instruments is attached. Request for any changes or alterations to the instrument should be sent via email to kamau.siwatu@ttu.edu. When using the instrument(s) please cite accordingly.

- Culturally Responsive Teaching Self-Efficacy Scale

Siwatu, K. O. (2007). Preservice teachers' culturally responsive teaching selfefficacy and outcome expectancy beliefs. Teaching and Teacher Education, 23, 1086-1101.

- Culturally Responsive Teaching Outcome Expectations Scale

Siwatu, K. O. (2007). Preservice teachers' culturally responsive teaching selfefficacy and outcome expectancy beliefs. Teaching and Teacher Education, 23, 1086-1101.

- Culturally Responsive Classroom Management Self-Efficacy Scale

Siwatu, K. O., Putnam, M., Starker, T. V., \& Lewis, C. (2015). The development of the culturally responsive classroom management self-efficacy scale: Development and initial validation. Urban Education. Prepublished September 9, 2015.

Best wishes with your research.

Sincerely,

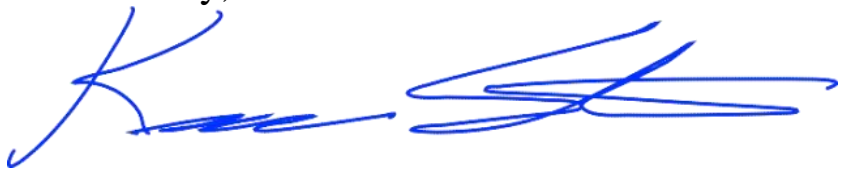

Kamau Oginga Siwatu, PhD

Professor of Educational Psychology

Box 41071 | Lubbock, Texas | 79409-1071 | T 806-834-5850 |F 806-742-2179

An EEO/Affirmative Action Institute 


\section{Appendix F}

\section{Written Reflection Exit Prompts}

- At what moment in today's meeting did you feel most engaged with what was happening?

- At what moment in today's meeting were you most distanced from what was happening?

- What action (or dialogue) that anyone took during the meeting did you find the most helpful?

- What action (or dialogue) that anyone took during the meeting did you find the most puzzling or confusing?

- What about the meeting surprised you the most?

- What would you like to have had occur in the meeting?

Adapted from Critical Incidence Protocol in Brookfield, S. (1995). Becoming a critically reflective teacher. San Francisco, CA: Jossey-Bass. 


\section{Appendix G}

\section{Initial Interview Questions}

1. In your own words, what is the purpose of PLC?

2. Thinking of the most productive or engaging PLCs you have participated in, what activities occurred and what did you find particularly useful or engaging?

3. Do the PLCs to which you currently participate regularly engage in reflection and how does it present?

4. In your own words, what is reflection (specifically regarding professional issues)?

5. How often and to what degree do you regularly engage in reflection?

6. How often and to what degree are you asked to or are personally motivated to examine your praxis and efficacy beliefs for assumptions and bias?

7. Thinking of the PLCs you have-now or in the past--participated in, describe the ways in which the PLC changed your praxis? 


\section{Appendix H}

\section{Final Interview Questions}

1. Thinking about your practice and routines over the past months, please describe any situations where you consciously tried out new strategies?

2. Over the past months, please describe any situations or circumstances when you reflected on your own practice either self-initiated or by external feedback.

3. Thinking about your practice during periods of any challenging implementations of new understandings over the past months, please describe any situations when you were actively struggling not to revert to old methods or fall back on old patterns.

4. Thinking back over the past months, please describe any situations or circumstances where you received, shared, and/or reflected on new ideas to implement.

5. Thinking about your practice in the past months, please describe any situations or circumstances when you perceived more awareness of either an existing idea or a new idea used in the classroom.

6. Thinking about your practice and routines over the past months, please describe any situations or circumstances when you perceived an intention to try a new practice, an intention to continue a new practice and/or the intention to continue an old practice.

7. Thinking about your practice over the past months, please describe any situations when you were aware that a new implementation was something you 
would continue to use or that you would permanently discard and revert to the old practice.

8. Describe any situations when you perceived a change in emotions because of a new practice implemented, positive (e.g., pride, satisfaction), negative emotion (e.g., irritation, fear, doubt), and/or surprise from an unexpected revelation. 


\section{Appendix I}

\section{Recruitment Document: Text for Flyer for Critical Reflection in PLC Study}

You are invited to join in a case study on the role of critical reflection in a PLC. This project is focused on how critical reflection unfolds within a PLC and its effects on teacher efficacy beliefs, teacher practice, and teacher learning.

\section{Participation:}

As a Phase I participant you will be asked to:

a. Complete two brief pre-interview questionnaires ( $15 \mathrm{~min} / \mathrm{each}$ )

b. Allow the researcher-participant to take field notes of the PLC processes

\section{As a Phase II participant you, in addition to Phase I requirements, will be asked to:}

a. Allow for two digitally recorded interviews with the researcher $(\approx 30 \mathrm{~min} / \mathrm{each})$

b. Respond to reflection prompts in writing after regular PLC meetings (5 min)

c. Participate in one small group meeting with $2-4$ other participants $(90 \mathrm{~min})$

d. Review typed transcripted records of your interviews and meetings for accuracy.

All interviews and small group meetings will be scheduled at times and places acceptable to you. Participation in these activities are voluntary.

\section{What you will be asked during the interviews?}

The interview questions will focus on learning more about:

Your understanding of PLCs and reflection, and what their purpose is regarding your praxis

Your perceptions of how PLCs and reflection have influenced your practice and learning.

\section{Timeline:}

January-June 2018 all activities

\section{Benefits:}


This study has the potential to highlight conditions conducive to teachers' learning and growth within learning communities. Results of this study of teachers' experiences with critical reflection may uncover areas for improvement by strengthening PLCs, teachers' learning, and promoting more positive student outcomes.

\section{Confidentiality:}

To safeguard the identity of participants, data will be kept in password protected and locked rooms. All documents, recordings, and transcripts will be strictly confidential and assigned a pseudonym and scrubbed for de-identification. All materials will be destroyed after three years.

\section{Who do I contact if I have questions about the case study?}

If you are interested in participating or have questions about the case study, please contact Patrizia Mastne: pmastne@pdx.edu or (503) 550-7550. 


\title{
Appendix J
}

\author{
ATLAS.ti@ Report \\ Code Categories
}

\section{Benefits of Communal Reflection}

Members:

- Benefits of CFG • Benefits of Communal Reflection $\bullet$ Benefits of PLC $\bullet$ Benefits of Reflection $\bullet$ CFG preference $\circ$ Changing Praxis $\bullet$ Community $\bullet$ Community building attempt $\bullet$ Disengaged $\bullet$ Effective PLC activities $\bullet$ Effective team teaching needs reflection • Efficacy $\bullet$ Emotional toll differs for POC • Engagement with PLC • Expertise of all • Frustration $\bullet$ Improving practice $\bullet$ Increase of reflecting time $\bullet$ Ineffective (Reflection)Strategy • Informal reflecting • Members on different pages • Microaggressions • Missed opportunities • Positive outcomes of effective PLC • Quality reflection needed $\bullet$ Raising awareness of new practice $\bullet$ Reflection as a communicative action vs just thinking $\bullet$ Reflection as Part of the job $\bullet$ Reflection purpose • Relevant Content $\bullet$ Rich discussions $\bullet$ Sharing expertise $\bullet$ Structured meeting for reflection $\bullet$ Student teacher $\bullet$ Superficial reflection $\bullet$ Teachers on automatic pilot $\bullet$ Team teaching $\bullet$ Trust $\bullet$ Trust lacking $\bullet$ Unstructured Reflection White fragility

\section{Benefits of PLC/CFG}

Members:

- Relevant Content $\bullet$ Benefits of CFG • Benefits of Communal Reflection • Benefits of PLC $\bullet$ Benefits of Reflection $\bullet$ CFG changed praxis $\bullet$ CFG preference $\bullet$ CFG Protocols $\circ$ Changing Praxis $\bullet$ Community $\bullet$ Community building attempt $\bullet$ Effective PLC activities • Efficacy • General consensus • history with learning communities • Ideal PLC • Improving practice $\bullet$ Increase of reflecting time $\bullet$ isolation $\bullet$ Limitation 
of the regular PLC $\bullet$ Long history $\bullet$ Positive outcomes of effective PLC $\bullet$ Problem of praxis $\bullet$ Quality reflection needed $\bullet$ Raising awareness of new practice $\bullet$ Reflection as a communicative action vs just thinking • Reflection purpose $\bullet$ Relevant Content Rich discussions $\bullet$ Sharing expertise $\bullet$ Structured meeting for reflection $\bullet$ Structured meeting needed $\bullet$ Trust

\section{Disengagement}

Members:

$\bullet$ Irrelevant Content $\bullet$ Confirmation of otherness $\bullet$ Crossed communication $\bullet$

Disengaged • Emotional toll differs for POC • Engaged to maintain barrier •

Frustration $\bullet$ Gaslighting $\bullet$ Ineffective PLC activity $\bullet$ isolation $\bullet$ Limitation of the regular PLC • Members on different pages • Microaggressions • Missed opportunities • No preparation $\bullet$ Norms not set, sanctity not preserved $\bullet$ Not a safe environment $\bullet$ On the Defense $\bullet$ PLC was unproductive $\bullet$ POC as token representative • Superficial reflection $\bullet$ Surprise over heated discussion $\bullet$ Trained facilitator lacking $\bullet$ Trust lacking $\bullet$ Unstructured $\bullet$ Venting vs reflecting $\bullet$ White fragility

\section{Praxis Improvement}

Members:

- Relevant Content $\bullet$ Benefits of CFG $\bullet$ Benefits of Communal Reflection $\bullet$ Benefits of PLC • Benefits of Reflection $\bullet$ CFG changed praxis $\bullet$ CFG preference $\bullet$ CFG Protocols $\circ$ Changing Praxis • Effective PLC activities • Efficacy • Improving practice $\bullet$ Quality reflection needed $\bullet$ Raising awareness of new practice $\bullet$ Reflection as a communicative action vs just thinking $\bullet$ Reverting to old praxis when challenged $\bullet$ Sharing expertise

\section{Teacher beliefs}


Members:

- Benefits of Communal Reflection $\bullet$ Benefits of Reflection $\bullet$ CFG changed praxis $\circ$ Changing Praxis • Community $\bullet$ Efficacy $\bullet$ Improving practice $\bullet$ Internally motivated to reflect $\bullet$ Missed opportunities $\bullet$ Quality reflection needed $\bullet$ Raising awareness of new practice $\bullet$ Range of emotions $\bullet$ Reflection as Part of the job $\bullet$ Self talk $\bullet$ Surprise over heated discussion $\bullet$ Teachers on automatic pilot $\bullet$ Trust 